\title{
Searches for Radio Pulsars \& Fast Transients and Multiwavelength Studies of Single-pulse Emission
}

\author{
Mitchell B. Mickaliger \\ West Virginia University
}

Follow this and additional works at: https://researchrepository.wvu.edu/etd

\section{Recommended Citation}

Mickaliger, Mitchell B., "Searches for Radio Pulsars \& Fast Transients and Multiwavelength Studies of Single-pulse Emission" (2013). Graduate Theses, Dissertations, and Problem Reports. 3657.

https://researchrepository.wvu.edu/etd/3657

This Dissertation is protected by copyright and/or related rights. It has been brought to you by the The Research Repository @ WVU with permission from the rights-holder(s). You are free to use this Dissertation in any way that is permitted by the copyright and related rights legislation that applies to your use. For other uses you must obtain permission from the rights-holder(s) directly, unless additional rights are indicated by a Creative Commons license in the record and/ or on the work itself. This Dissertation has been accepted for inclusion in WVU Graduate Theses, Dissertations, and Problem Reports collection by an authorized administrator of The Research Repository @ WVU.

For more information, please contact researchrepository@mail.wvu.edu. 


\section{Searches for Radio Pulsars \& Fast Transients and Multiwavelength Studies of Single-pulse Emission}

\section{Mitchell B. Mickaliger}

Dissertation submitted to the Eberly College of Arts and Sciences at West Virginia University in partial fulfillment of the requirements for the degree of

Doctor of Philosophy in Physics

\section{Duncan Lorimer, Ph.D., Chair Maura McLaughlin, Ph.D. D.J. Pisano, Ph.D. Wathiq Abdul-Razzaq, Ph.D.} Phil Turk, Ph.D.

Department of Physics

Morgantown, West Virginia 2013

Keywords: Pulsars; Neutron Stars Copyright 2013 Mitchell B. Mickaliger 


\title{
ABSTRACT \\ Searches for Radio Pulses \& Fast \\ Transients and Multiwavelength \\ Studies of Single-pulse Emission
}

\author{
Mitchell B. Mickaliger
}

Pulsars are excellent tools for studying a wide array of astrophysical phenomena (e.g. gravitational waves, the interstellar medium, general relativity), yet they are still not fully understood. What are their emission processes and how do they change at different energies? How is giant pulse emission different from regular emission? How are different classes of pulsars (RRATs, magnetars, nulling pulsars, etc.) related? Answering these questions will not only help us to understand pulsars in general, but will also help improve techniques for pulsar searches and timing, gravitational wave searches, and single-pulse searches. The work we present here aims to answer these questions through studies of giant pulse emission, the discovery of new pulsars, and single-pulse studies of a large population of pulsars and RRATs.

We took advantage of open telescope time on the 43-m telescope in Green Bank, WV to conduct a long-term study of giant pulses from the Crab pulsar at 1.2 $\mathrm{GHz}$ and $330 \mathrm{MHz}$. Over a timespan of 15 months, we collected a total of 95000 giant pulses which we correlated with both $\gamma$-ray photons from the Fermi satellite and giant pulses collected at $8.9 \mathrm{GHz}$. Statistics of these pulses show that their amplitudes follow power-law distributions, with indices in the range of 2.1 to 3.1. The correlation with giant pulses at $8.9 \mathrm{GHz}$ showed that the emission processes at $1.2 \mathrm{GHz}$ and $8.9 \mathrm{GHz}$ are related, despite significant profile differences. The correlation with Fermi $\gamma$-ray photons was to test if increased pair production in the magnetosphere was the cause of giant pulses. Our findings suggest that, while it may play a role, increased pair production is not the dominant cause of giant pulses.

As part of a single-pulse study, we reprocessed the archival Parkes Multibeam Pulsar Survey, discovering six previously unknown pulsars. PSR J0922-52 has a period of $9.68 \mathrm{~ms}$ and a DM of $122.4 \mathrm{pc} \mathrm{cm}^{-3}$. PSR J1147-66 has a period of $3.72 \mathrm{~ms}$ and a DM of $133.8 \mathrm{pc} \mathrm{cm}^{-3}$. PSR J1227-6208 has a period of $34.53 \mathrm{~ms}$, a DM of $362.6 \mathrm{pc} \mathrm{cm}^{-3}$, is in a 6.7 day binary orbit. PSR J1546-59 has a period of $7.80 \mathrm{~ms}$ and a DM of $168.3 \mathrm{pc} \mathrm{cm} \mathrm{cm}^{-3}$. PSR J1725-3853 is an isolated $4.79-\mathrm{ms}$ pulsar with a DM of $158.2 \mathrm{pc} \mathrm{cm}^{-3}$. PSR J1753-2822 has a period of $18.62 \mathrm{~ms}$, a DM of $298.4 \mathrm{pc} \mathrm{cm}^{-3}$, and is in a 9.3 hour binary orbit. These pulsars were likely missed in earlier processing efforts due to the fact that they have both high DMs and short periods, and also the large number of candidates that needed to be looked through. These discoveries suggest that further pulsars are awaiting discovery in the multibeam survey data. 
We also searched for single pulses out to a DM of $5000 \mathrm{pc} \mathrm{cm}^{-3}$ with widths of up to two seconds in our reprocessing of the PMPS data. We recorded single pulses from 264 known pulsars and 15 RRATs. We fit amplitude distributions of the pulsars with lognormal distributions and power-law tails, finding that some pulsars show a deviation from a lognormal distribution in the form of an excess of high-energy pulses. Fitting lognormal distributions to the amplitudes of pulses from RRATs showed similar behavior for most RRATs. Here, however, there seem to be two distinct populations of pulses, with the first population being consistent with noise. For pulsars that were detected in a periodicity search, we computed the ratio of their single-pulse S/N to their FFT S/N and looked for correlations between this ratio and physical parameters of the pulsars. We found a few strong correlations, but they all seem to be due to the strongest correlation between the ratio and spin period. 


\section{Acknowledgments}

The third time really is the charm. After much blood, sweat, and tears I have finally made it to this point. There is absolutely no way I would have gotten here without the help of an innumerable amount of people. They say it takes a village, and I really have had the support of a great deal of people, both in and out of the Physics Department.

First and foremost, I have to thank my advisors, Duncan Lorimer and Maura McLaughlin, for all the time and effort they put into helping me with my research. There were so many simple things, like Linux, that I had no experience with when I arrived here, and thanks to my advisors, I have become quite the expert in most of these things over these past six years.

I also have to thank my other committee members, D.J. Pisano, Wathiq AbdulRazzaq, and Phil Turk, for their extremely useful discussions, both about this thesis and life in general.

I would be hard-pressed not to acknowledge the many people throughout the Physics Department, and select other departments, who not only listened to my rantings, but also gave me much needed advice. It proved invaluable to both my career and peace of mind.

I certainly need to thank my office mates, and honestly the majority of the Annex, for putting up with me. Josh, Pete, Katie, Andrew, Spencer, Sam, Nate, and all of the rest, you were more helpful than you probably know. I cannot forget to thank my long-distance office mates on the third floor (Luke, Jerry, Colin, Dusty), some of whom I have spent so much time with one might think I lived with them.

The office staff, especially Sherry, proved invaluable over the years. They were able, and more than willing, to help me navigate the paperwork and bureaucracy that piled up far too often. My sanity would have quickly dissolved without them.

I am blessed to have many close friends outside of the department as well. Art, Tracy, Judy, thank you for helping me forget my sorrows. To my second family at the Elks Lodge \#411, I cannot thank you enough. You were all very supportive of me throughout the years.

I need to especially thank Amy and Andrew, who continue to support me from afar. You guys were always there for me and Kristi when we needed to get away. You did far too much for us, and there is no way we could ever fully pay you back.

My parents and sister have also been exceedingly helpful, even when times were tough for them. Thank you for listening, for all the support over the years, and for your enthusiasm in what I do. Your pride in me helps me get out of bed on those days when I want to do otherwise.

Finally, infinite thanks to my beautiful, loving, supportive, caring wife Kristi, who had to tolerate me as I went through this arduous task. Not only did you put up with my stress and anxiety, you did all of those things around the house that I stopped doing, and you bent over backwards to cater to my needs, driving me to and from work at all hours of the day and night when my car broke and I did not have time to fix it. I love you.

I am dedicating this work to my grandfather, Ben, who was so proud of me and constantly told this fact to everyone who would listen. 


\section{Table of Contents}

List of Tables $\quad$ vii

List of Figures viii

List of Abbreviations and Symbols $\quad$ ix

1 Introduction 1

1.1 Violent Beginnings . . . . . . . . . . . . . . . . 2

1.2 Emission Mechanism ................... . . . . . . . . . . . . .

1.2.1 Giant Pulses . . . . . . . . . . . . . . . . . . 7

1.3 The Evolution of Pulsars . . . . . . . . . . . . . . . . . 7

1.3.1 Rotating Radio Transients . . . . . . . . . . . . . . . . . 9

1.4 Pulsar Searching . . . . . . . . . . . . . . . . . . 11

1.4.1 Radio Frequency Interference . . . . . . . . . . . . . . . . 12

1.4.2 Dedispersion . . . . . . . . . . . . . . . . . . 13

1.4.3 Frequency Domain Searches ............... 15

1.4.3.1 Acceleration Searches ........... . . 16

1.4.3.2 Candidate Selection . . . . . . . . . . . . 18

1.5 Pulsar Timing . . . . . . . . . . . . . . . . . . 20

1.6 Outline of Thesis . . . . . . . . . . . . . . 25

2 A Giant Sample of Giant Pulses from the Crab Pulsar 26

2.1 Introduction . . . . . . . . . . . . . . . . . . 26

2.2 Radio Observations . . . . . . . . . . . . . . . . . . . 29

2.3 Amplitude Distributions . . . . . . . . . . . . . . . . . . . 34

2.4 Correlating Radio GPs from the GB43 and GBT . . . . . . . . . . . 37

2.5 Correlating Radio GPs from the GB43 with $\gamma$-ray Photons from Fermi 39

2.6 Crab Nebula $\gamma$-ray Flare . . . . . . . . . . . . . . . . . . . 42

2.7 Conclusions . . . . . . . . . . . . . . . . . . . . 43

3 Discovery of Six New Pulsars in Archival Data 63

3.1 Introduction . . . . . . . . . . . . . . . . 63

3.2 Data Reduction . . . . . . . . . . . . . . . . . . 64

3.3 Newly Discovered Pulsars . . . . . . . . . . . . . . . . . . 65

3.3.1 PSR J0922-52 . . . . . . . . . . . . . . . . . . . 66

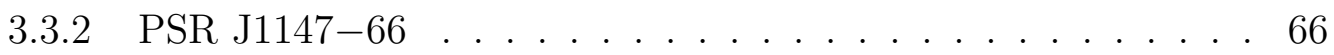

3.3.3 PSR J1227-6208 . . . . . . . . . . . . . . . . . . . 67

3.3.4 PSR J1546-59 . . . . . . . . . . . . . . . . . . . . . . 68

3.3.5 PSR J1725-3853 . . . . . . . . . . . . . . . . . . . 68

3.3.6 PSR J1753-2822 . . . . . . . . . . . . . . . . . . 70

3.4 Conclusions . . . . . . . . . . . . . . . . . . . . 71 
4 A Study of Single Pulses in the Parkes Multibeam Pulsar Survey 76

4.1 Introduction . . . . . . . . . . . . . . . 76

4.2 Data Reduction . . . . . . . . . . . . . . . . . . . 79

4.3 Known Pulsars . . . . . . . . . . . . . . . . . . . 82

4.3.1 Energy Distributions . . . . . . . . . . . . . . . . 83

4.3.2 Comparison of $\frac{\mathrm{S} / \mathrm{N}_{\mathrm{SP}}}{\mathrm{S} / \mathrm{N}_{\mathrm{FFT}}}$ Ratio to Physical Properties . . . . . 85

4.4 RRATs . . . . . . . . . . . . . . . . . . . . 88

4.5 Conclusions . . . . . . . . . . . . . . . . . . . . . 89

5 Conclusions 111

5.1 The Crab Pulsar . . . . . . . . . . . . . . . . . . 111

5.2 New Pulsars from the Parkes Multibeam Pulsar Survey . . . . . . . . 112

5.3 Single-pulse Studies of the PMPS . . . . . . . . . . . . . . . . 113 


\section{List of Tables}

2.1 Observational Parameters of Crab Study . . . . . . . . . . . . . 56

2.2 Various Timescales Associated with Different Observing Bands . . . . 58

2.3 Differential Power-law Measurements for the MP and IP . . . . . . . 59

2.4 Comparison of Differential Power-law Indices and Previously Published Values . . . . . . . . . . . . . . . . . . 61

2.5 Comparison of Differential Power-law Indices and Those of Different Source Classes . . . . . . . . . . . . . . . . . . . 61

2.6 Maximum Correlation for Each Frequency and Energy Cut . . . . . . 62

3.1 Observational Parameters for PSR J1725-3853 . . . . . . . . . . . . 74

3.2 Timing and Derived Parameters for PSR J1725-3853 . . . . . . . . . 74

3.3 Timing and Derived Parameters for PSR J1753-2822 . . . . . . . . . 75

4.1 Single-pulse Detections of Known Pulsars . . . . . . . . . . . . . . . . 102

4.2 Correlation Between $\frac{\mathrm{S} / \mathrm{N}_{\mathrm{SP}}}{\mathrm{S} / \mathrm{N}_{\mathrm{FFT}}}$ and Physical Characteristics . . . . . . . 109

4.3 Single-pulse Detections of RRATs . . . . . . . . . . . . . . . 110 


\section{List of Figures}

1.1 Toy model of a pulsar. . . . . . . . . . . . . . . . . . . 6

1.2 Train of bright, single pulses. . . . . . . . . . . . . 8

1.3 P- $\dot{P}$ diagram. . . . . . . . . . . . . . . . . . . . . . . . . 9

1.4 Example of Single-Pulse Search Diagnostic Plot . . . . . . . . . . . . 10

1.5 Example of Periodicity Search Diagnostic Plot . . . . . . . . . . . . . 20

1.6 Example of Timing Residuals . . . . . . . . . . . . . . 23

2.1 Comparison of Crab Folded Profiles at $330 \mathrm{MHz}, 350 \mathrm{MHz}$, and $\gamma$-ray

Energies . . . . . . . . . . . . . . . . . . . 46

2.2 Number of Crab GPs vs Pulse Phase . . . . . . . . . . . . . . . . . 47

2.3 Single GP Observed at $330 \mathrm{MHz}$ and $350 \mathrm{MHz}$. . . . . . . . . . . . . 48

2.4 Average Crab Folded Profile at $330 \mathrm{MHz}$ and $1.2 \mathrm{GHz}$. . . . . . . . 49

2.5 Average Crab MP and IP GP Profiles . . . . . . . . . . . . . . . . . 50

2.6 Example of Power-law Fit to Amplitude Distribution . . . . . . . . . 51

2.7 Average Crab Folded Profile at $1.2 \mathrm{GHz}$ and $9.8 \mathrm{GHz}$. . . . . . . . . 52

2.8 Strength of MP vs IP in the Crab Folded Profile . . . . . . . . . . . . 53

2.9 Correlation Between GPs and Fermi Photons . . . . . . . . . . . . . 54

2.10 Crab Folded Profile and GP Comparison Before and After $\gamma$-ray Flare 55

3.1 Folded Profiles of Confirmed Pulsars _ . . . . . . . . . . . . . . 73

4.1 Example of Single-Pulse Search Diagnostic Plot . . . . . . . . . . . . 92

4.2 Examples of Lognormal Fits to Amplitude Distributions . . . . . . . 93

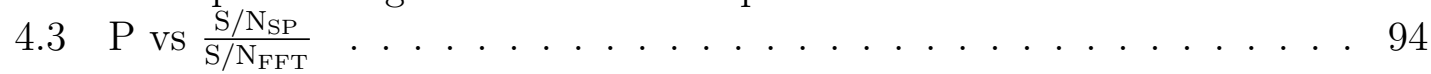

$4.4 \dot{P} \mathrm{VS} \frac{\mathrm{S} / \mathrm{N}_{\mathrm{SP}}}{\mathrm{S} / \mathrm{N}_{\mathrm{FFT}}} \ldots \ldots \ldots \ldots \ldots \ldots$

$4.5 \mathrm{DM} v \mathrm{vs} \frac{\mathrm{S} / \mathrm{N}_{\mathrm{SP}}}{\mathrm{S} / \mathrm{N}_{\mathrm{FFT}}} \ldots \ldots \ldots \ldots \ldots \ldots . \ldots \ldots . \ldots \ldots$

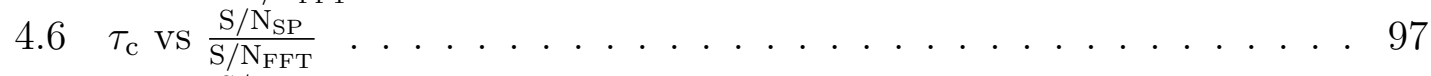

$4.7 \dot{E} \mathrm{vS} \frac{\mathrm{S} / \mathrm{N}_{\mathrm{SP}}}{\mathrm{S} / \mathrm{N}_{\mathrm{FFT}}} \ldots \ldots \ldots \ldots \ldots \ldots$

$4.8 B_{\mathrm{surf}} \mathrm{vS} \frac{\mathrm{S} / \mathrm{N}_{\mathrm{SP}}}{\mathrm{S} / \mathrm{N}_{\mathrm{FFT}}} \ldots \ldots \ldots \ldots \ldots \ldots \ldots$

$4.9 \quad B_{\mathrm{LC}}$ vs $\frac{\mathrm{S} / \mathrm{N}_{\mathrm{SP}}}{\mathrm{S} / \mathrm{N}_{\mathrm{FFT}}} \ldots \ldots \ldots \ldots \ldots$

4.10 Examples of Lognormal Fits to RRAT Amplitude Distributions . . 101 


\section{List of Abbreviations and Symbols}

$\begin{array}{ll}\nu & \text { Rotational Frequency } \\ \dot{\nu} & \text { Rotational Frequency Derivative } \\ \phi & \text { Pulse Phase } \\ \tau_{\mathrm{c}} & \text { Characteristic Age } \\ & \\ b & \text { Galactic Latitude } \\ B & \text { Magnetic Field } \\ B_{\mathrm{LC}} & \text { Magnetic Field at the Light Cylinder } \\ B_{\text {surf }} & \text { Surface Magnetic Field } \\ \mathrm{c} & \text { Speed of Light } \\ \mathrm{DM} & \text { Dispersion Measure } \\ \dot{E} & \text { Spin-down Luminosity } \\ \mathrm{FFT} & \text { Fast Fourier Transform } \\ \mathrm{GHz} & \text { Gigahertz } \\ \mathrm{GP} & \text { Giant Pulse } \\ \mathrm{GUPPI} & \text { Green Bank Ultimate Pulsar Processing Instrument } \\ \mathrm{IP} & \text { Crab Pulsar Interpulse } \\ \mathrm{ISM} & \text { Interstellar Medium } \\ \mathrm{Jy} & \text { Jansky } \\ \mathrm{kpc} & \text { Kiloparsec } \\ l & \text { Galactic Longitude } \\ \mathrm{lt}-\mathrm{s} & \text { Light Second } \\ M \odot & \text { Mass of the Sun } \\ \mathrm{MHz} & \text { Megahertz } \\ \mathrm{MJD} & \text { Modified Julian Day } \\ \mathrm{MP} & \text { Crab Pulsar Main Pulse } \\ \mathrm{MSP} & \text { Millisecond Pulsar } \\ n_{\mathrm{e}} & \text { Electron Number Density } \\ P & \text { Rotational Period } \\ \dot{P} & \text { Rotational Period Derivative } \\ \mathrm{pc} & \text { Parsec } \\ \mathrm{PMPS} & \text { Parkes Multibeam Pulsar Survey } \\ \mathrm{PSR} & \text { Pulsar } \\ \mathrm{RFI} & \text { Radio Frequency Interference } \\ \mathrm{RRAT} & \text { Rotating Radio Transient } \\ \mathrm{S} / \mathrm{N} & \text { Signal-to-Noise Ratio } \\ \mathrm{t}_{\mathrm{DM}} & \text { DM Smearing } \\ \mathrm{t}_{\mathrm{eff}} & \text { Effective Resolution } \\ \mathrm{t}_{\text {samp }} & \text { Sampling Time } \\ \text { scatt } & \text { Scattering Time } \\ & \end{array}$




\section{Chapter 1}

\section{Introduction}

The Universe is filled with transients, sources which lack steady-state emission. Many of these transients are well studied, but still not fully understood. Transients include ultra-high energy particles, the Sun, planets, brown dwarfs, flare stars, X-ray binaries, soft $\gamma$-ray repeaters, maser flares, active galactic nuclei, radio supernovae, $\gamma$-ray bursts, annihilating black holes, and extraterrestrial transmitters (Cordes et al., 2004). One example of the transient phenomenon are pulsars. Due to their transient nature, pulsars were not discovered until relatively recently, just under 50 years ago in 1967, and quite by accident. While looking at some data she had taken as part of a survey at Cambridge to study the scintillation of extragalactic radio sources, Jocelyn Bell found sources which emitted individual pulses with durations (pulse widths) of 10s of milliseconds of what at first was thought to be man-made interference (Hewish et al., 1968). After an exciting flurry of hypotheses as to the origin of this signal, including extraterrestrial life, it was finally concluded that the source of the signal must originate from a neutron star, which had been theorized just 30 years before (Baade \& Zwicky, 1934). The commonly accepted picture, which we will expand upon below, is the so-called lighthouse model (Gold, 1968; Pacini, 1968) in which the observed pulses are produced by a radiation beam originating from the neutron star's spinning magnetic poles. The pulse repetition 
period matches the rotation period of the neutron star. Even though there are still many open questions about pulsars, they have been shown to be outstanding celestial clocks and are actively being used to study a vast array of astrophysical phenomena, including gravitational waves, the interstellar medium, and general relativity (Jenet et al., 2009; Ferdman et al., 2010; Hobbs et al., 2010; Manchester et al., 2013; Taylor \& Weisberg, 1982; Kramer et al., 2006; Antoniadis et al., 2013). The goal of this chapter is to review the main properties and physical processes pertaining to pulsars that will be relevant for the rest of this thesis.

\subsection{Violent Beginnings}

We now know that pulsars are born from supernovae, due to the discovery of the Crab pulsar with a period of $33 \mathrm{~ms}$ (Staelin \& Reifenstein, 1968) in a remnant of a supernova seen nearly 1000 years ago by Chinese astronomers (Stephenson \& Green, 2002). As the core of a massive star collapses into a neutron star, the magnetic field is intensified, leaving the resulting pulsar with a canonical surface magnetic field of $10^{12}$ Gauss. Angular momentum is also conserved in the collapse, leading to a very high initial rotation frequency for the pulsar. Although the first pulsar discovered by Bell had a rotation period of roughly one second, most pulsars have spin periods on the order of a few hundred milliseconds, with some as low as only a few milliseconds, like the fastest spinning pulsar currently known, PSR J1748-2446ad, with a spin period of $1.39 \mathrm{~ms}$ (Hessels et al., 2006). The longest period pulsars have observed periods of up to eight seconds. In general, pulsars 
which have spin periods anywhere from roughly 20 milliseconds to eight seconds are considered 'normal' pulsars, while those with periods of less than 20 milliseconds are dubbed 'millisecond' pulsars $(\mathrm{MSPs})^{1}$.

\subsection{Emission Mechanism}

When a pulsar is born, it generally has a period of $\sim 100$ milliseconds, although some are thought to be born with periods of only a few tens of milliseconds. For example, extrapolation of the Crab pulsar's current period back to its birth in the supernova explosion results in an initial period of $16 \mathrm{~ms}$ (see, e.g., Glendenning, 1996). Faucher-Giguère \& Kaspi (2006), through simulations based on survey results, found that the average period at birth is 300 milliseconds, with a standard deviation of 150 milliseconds. Based on X-ray observations of extragalactic supernovae, Perna et al. (2008) state that no more than a small fraction of the total pulsar population can be born with millisecond spin periods, specifically periods less than 40 milliseconds $^{2}$. Like most celestial objects, the rotation and magnetic axes of the precursor star, and hence the pulsar itself, are offset. According to electrodynamics (see, e.g., Jackson, 1962), a rotating magnetic dipole will lose energy at a rate

$$
\dot{E}_{\text {dipole }}=\frac{2}{3 c^{3}}|m|^{2} \Omega^{4} \sin ^{2} \alpha .
$$

\footnotetext{
${ }^{1}$ Stricter definitions for normal and millisecond pulsars will be discussed later.

${ }^{2}$ In this regard, the Crab pulsar seems to be the exception in this regard rather than the rule.
} 
Here $m$ is the magnetic dipole moment, $\Omega$ is the rotation frequency, $\alpha$ is the angle between the magnetic moment and the spin axis, and $c$ is the speed of light. According to this equation, the greater the value of $\alpha$, the more intense the dipole radiation. This radiation will cause the pulsar to lose energy, thereby slowing its rotation rate.

There is yet another factor that contributes, somewhat, to the spin-down of pulsars. Since the surface magnetic field is so strong, and the pulsar is spinning so fast, there is an electric field induced near the surface of the pulsar, shown by Goldreich \& Julian (1969) to be

$$
E_{\|}=\frac{\Omega B_{\mathrm{S}} R}{c} \cos ^{3} \theta
$$

Here $B_{\mathrm{S}}$ is the strength of the magnetic field at the neutron star surface, $R$ is the assumed radius of the neutron star, and $\theta$ is the polar coordinate of the pulsarcentered coordinate system. This electric field is strong enough to pull particles from the surface of the star. This plasma then fills the magnetic field. The magnetic field, feeling the same electromagnetic force as the neutron star itself, rotates rigidly with the star. At some radius, though, the plasma-filled magnetic field approach the speed of light. The radius at which they would theoretically reach this speed is known as the light cylinder. Magnetic field lines that extend outside of this region cannot remain connected, and are therefore open, connecting with ambient magnetic fields around the pulsar. A simplified version of this can be seen in the toy model of a pulsar, shown in Figure 1.1. As the electrons spiral around the magnetic field 
lines, some are accelerated along the open field lines, carrying energy away from the pulsar.

The surface of the pulsar where these open field lines originate is designated the polar cap, which is centered on the magnetic axis. As electrons stream out along the open field lines in this small region, they emit synchrotron radiation. This results in a narrow, cone-shaped beam of radio emission. If we assume this cone is bounded by the closed field lines, then the width of the beam will be dependent upon the height above the surface of the pulsar at which the radiation is emitted. If this beam happens to sweep past our line of sight, we will see a pulse of radio light, much like a lighthouse. The observed beam width, then, will depend on how our line of sight intersects the beam. In order to achieve this emission, the plasma density must be much larger than the density expected in the magnetosphere. However, due to the loss of electrons above the polar cap, a polar 'gap' is created, resulting in a large electric field. This electric field then accelerates particles to relativistic energies. As they stream along the magnetic field lines, they produce curvature radiation and also inverse Compton scatter low energy photons. This emission is seen at high frequencies, in the $\gamma$-ray regime of the electromagnetic spectrum. Due to the strong magnetic field, the $\gamma$-ray photons are able to create electron-positron pairs. These particles produce further photons, which in turn produce more particles, leading to the large increase in density necessary for radio emission. These electron-positron pairs, created by this pair cascade, then produce the observed synchrotron radiation (Goldreich \& Julian, 1969). 


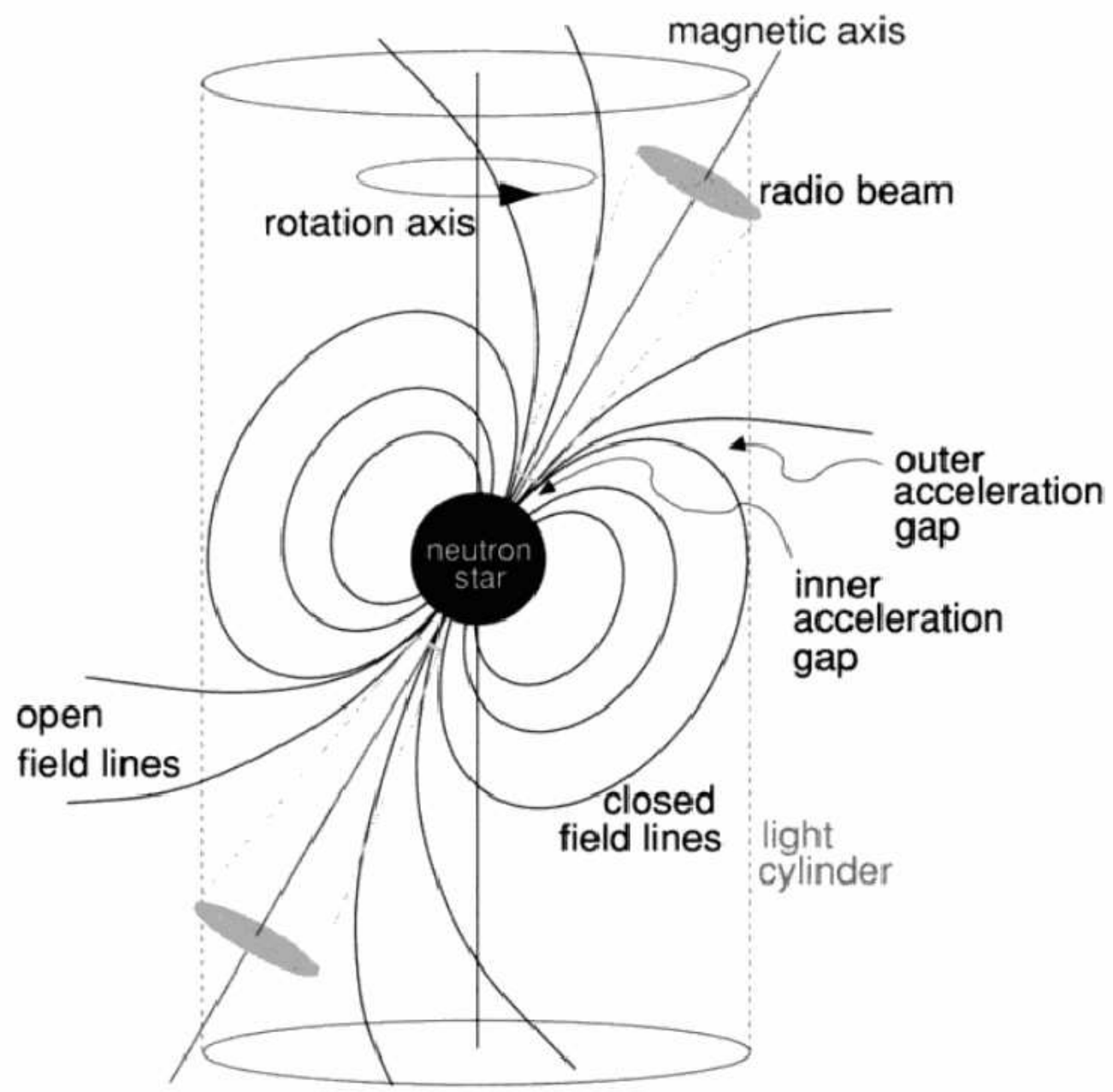

Figure 1.1: Toy model of a pulsar, consisting of a rotating neutron star with a dipolar magnetic field. The magnetic and spin axes are offset, causing us to observe pulses of emission if we are in the path of the radio beam. When the rigidly co-rotating magnetic field approaches the speed of light, the field lines can no longer remain closed. This boundary between open and closed field lines is defined as the light cylinder. Figure used with permission from the Handbook of Pulsar Astronomy (Lorimer \& Kramer, 2004). 


\subsubsection{Giant Pulses}

As one can see from Figure 1.2, pulse amplitudes vary from pulse to pulse. For some pulsars, the strongest pulses can be up to 1000 times stronger than the average pulse. These phenomena, dubbed 'giant' pulses, seem to differ from normal pulsar emission, in that their amplitudes follow a power-law (e.g., Argyle \& Gower, 1972; Popov \& Stappers, 2007), while the single-pulse amplitudes from normal pulsars follow a lognormal distribution (Ritchings, 1976). Hankins et al. (2003) found that giant pulses from the Crab pulsar are made up of many smaller pulses with pulse widths on the order of a couple of nanoseconds. These nanopulses show strong circular polarization, and switch between right- and left-handed polarizations rapidly. The enormous brightness temperatures of these nanopulses, on the order of $10^{37} \mathrm{~K}$, suggests a coherent source of emission. Hankins et al. (2003) also argue that the short timescale of the emission can only be explained by the collapse of wavepackets in the magnetosphere. The short emission timescales also constrain the region of emitting plasma to be no larger than one meter. Other recent theories attempting to explain the giant pulse emission mechanism include anomalous cyclotron resonances (Lyutikov et al., 1999; Machabeli \& Usov, 1979) and increased particle density in the magnetosphere due to reconnection (Lyutikov, 2007).

\subsection{The Evolution of Pulsars}

As mentioned before, pulsars are observed to have a range of spin periods. However, pulsars are usually not 'born' with spin periods of only a few milliseconds. 


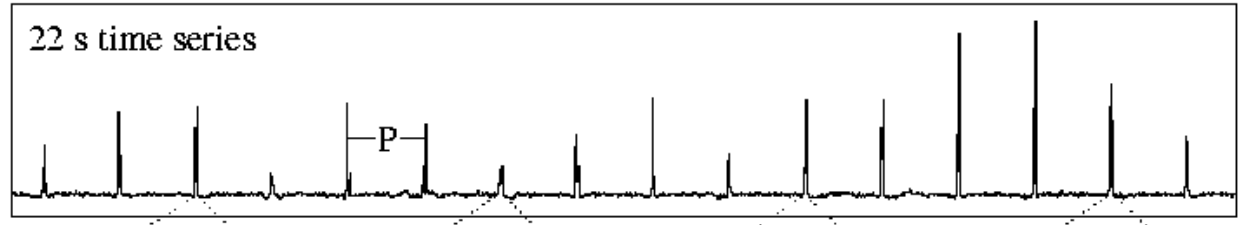

Figure 1.2: A series of bright, single pulses showing the variation in pulse amplitude. Figure used with permission from the Handbook of Pulsar Astronomy (Lorimer \& Kramer, 2004).

Spin-down from energy loss due to dipole radiation and kinetic energy lost to accelerated particles will then cause their periods to lengthen. It therefore seems to be a mystery as to why we see pulsars with periods of only a few milliseconds.

Of the more than 2200 pulsars known, 213 are found in binary systems. The majority of these systems, 161 in total, have pulsars with spin periods of only a few milliseconds. This correlation is not a coincidence. These MSPs in binary systems usually have companions with low masses, meaning they have already evolved. Pulsars in binary systems with higher mass, unevolved companions tend to have longer spin periods. During the evolution of the companion star, matter is accreted onto the pulsar (Bhattacharya \& van den Heuvel, 1991). As the matter spirals inward in an accretion disk, angular momentum is conserved. The matter that is deposited onto the surface of the neutron star acts to spin it up, dramatically increasing its rotation frequency.

When pulsars are born, in addition to spin periods on the order of $\sim 100$ milliseconds, their magnetic fields are also quite large $\left(10^{12}\right.$ Gauss $)$. The rate of change of their periods is generally around $10^{-15} \mathrm{ss}^{-1}$. Those pulsars which accrete matter from a companion and are spun up are found to have much smaller magnetic field strengths, on the order of $10^{8}$ Gauss, due to the magnetic field being somehow 


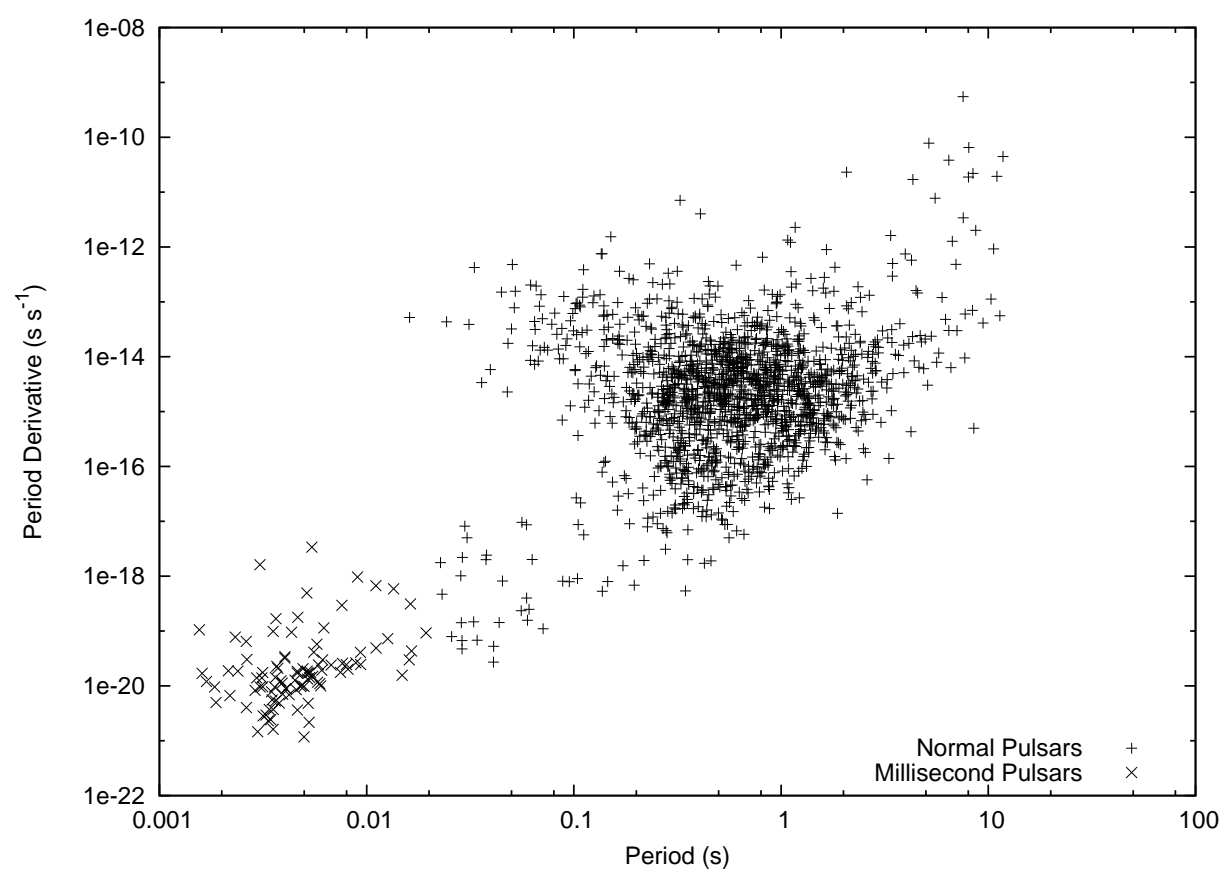

Figure 1.3: Spin periods of pulsars versus their period derivatives. There are two distinct categories of pulsars visible: normal pulsars and millisecond pulsars. Normal pulsars have periods above 20 milliseconds and period derivatives around $10^{-15} \mathrm{ss}^{-1}$, while millisecond pulsars generally have spin periods less than 20 milliseconds and period derivatives around $10^{-20} \mathrm{ss}^{-1}$.

'buried' by the infalling matter (Bisnovatyi-Kogan \& Komberg, 1974; Shibazaki et al., 1989). This reduction in the magnetic field of the pulsar will cause it to lose less energy through dipole radiation (see Equation 1.1). The resulting period derivatives of these MSPs tends to be $10^{-20} \mathrm{ss}^{-1}$. If the period and period derivatives of normal pulsars and MSPs are plotted, as in Figure 1.3, it can clearly be seen that the two populations inhabit two distinct sections of the diagram.

\subsubsection{Rotating Radio Transients}

While pulsars are known for their generally reliable nature, usually being detectable in a given observation, there is another type of neutron star which does not 

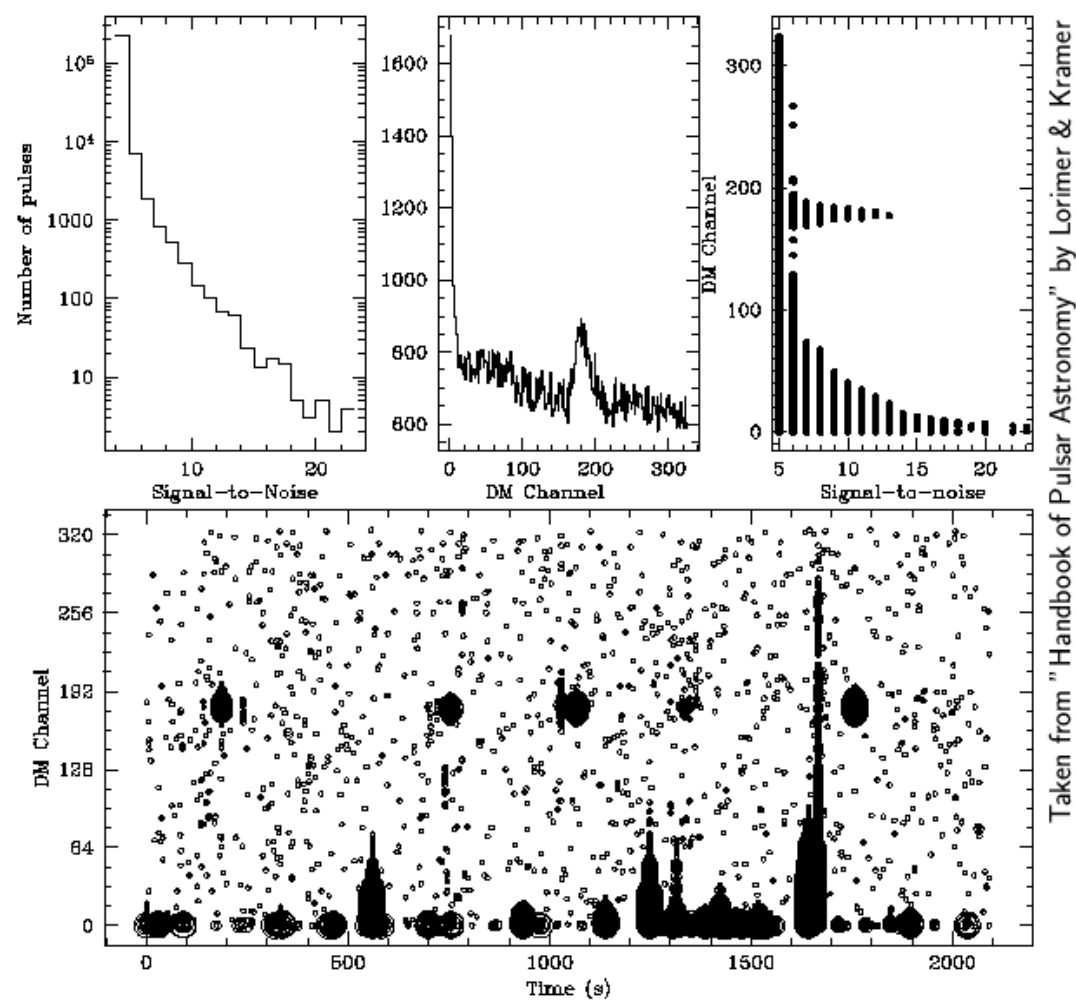

Figure 1.4: Example of a single-pulse plot, showing bright single pulses from PSR J1624-4616 around DM channel 192. RFI is also present, peaking at a DM of $0 \mathrm{pc} \mathrm{cm}^{-3}$. Figure used with permission from the Handbook of Pulsar Astronomy (Lorimer \& Kramer, 2004).

behave so predictably. These objects, called Rotating RAdio Transients, or RRATs, only seem to emit pulses sporadically. These were discovered by McLaughlin et al. (2006) and have typical burst rates of anywhere from one per minute to less than one per hour (Keane et al., 2011). Since they lack periodicity, they are not readily found by the standard method used to find pulsars. Instead, they are detected through visual inspection of a single-pulse plot, a plot of dispersion measure (DM, the integrated column density of free electrons along the line of sight) versus time. An example of such a plot is shown in Figure 1.4.

Sometimes enough pulses from a RRAT are seen together to calculate a period, 
but that is not always the case. Those RRATs for which a period and period derivative can be measured generally populate the 'island' of normal pulsars seen in Figure 1.3. After their initial discovery, some RRATs were found to be normal pulsars, albeit pulsars whose radio emission was very weak. In this case, only the few strongest pulses were detected initially. Weltevrede et al. (2006) showed that some pulsars, like PSR B0656+14, display pulses which cover a wide distribution of energies, including some bursts which are extremely powerful. They argue that, if located 12 times farther away, only these brightest bursts would be detectable, and this pulsar would be classified as a RRAT. Given how near this pulsar is (0.288 $\mathrm{kpc}$ ) and the distances to the original RRATs $(2-6.5 \mathrm{kpc})$, these RRATs could easily be pulsars if they displayed similar pulse modulation (Keane \& McLaughlin, 2011). Other proposed scenarios for the sporadic emission from RRATs include interactions between a neutron star and a surrounding debris disk (Li, 2006), bursts of emission due to disrupted plasma in radiation belts (Luo \& Melrose, 2007), and the interaction of a pulsar magnetosphere with a circumpulsar asteroid belt (Cordes \& Shannon, 2008).

\subsection{Pulsar Searching}

As mentioned above, there are different processes to find the different types of pulsars. These methods, as well as the difficulties associated with these methods, are discussed here. Pulsar searching first requires the signal from the pulsar to be 
recorded. Data are taken with some sampling time across a wide bandwidth ${ }^{3}$. To allow the removal of frequency dispersion due to propagation through the ionized medium, this band is broken up into discrete frequency channels which are digitized before being recorded to disk to perform the analysis described below.

\subsubsection{Radio Frequency Interference}

Pulsars are normally detected through their radio emission. However, there are an increasing number of terrestrial signals at the radio frequencies at which pulsars are normally observed. This makes separating the signal of a pulsar from this "radio frequency interference" (RFI) difficult. One way to mitigate the effects of RFI is to remove data which are dominated by it. Since pulsars are relatively weak sources, any data in which a very strong signal is present, e.g., Figure 1.4, can be assumed to be RFI and removed. However, another way to distinguish astrophysical signals from terrestrial signals, which does not rely on the removal of data, utilizes the interstellar medium (ISM). The ISM consists of ionized hydrogen, a plasma of ions and electrons. The electrons in this plasma act to delay the propagation of electromagnetic radiation traveling through them. This time delay, $\Delta t$, is frequency dependent, with lower frequencies being delayed more. Lorimer \& Kramer (2004) derive this delay to be

$$
\Delta t=4.15 \times 10^{6} \mathrm{~ms} \times\left(f_{1}^{-2}-f_{2}^{-2}\right) \times \mathrm{DM},
$$

\footnotetext{
${ }^{3}$ Sampling times can range from a few microseconds to hundreds of microseconds, with some as short as nanoseconds. Bandwidths range from a tens of $\mathrm{MHz}$ up to almost a $\mathrm{GHz}$.
} 
where $f_{1}$ and $f_{2}$ are the two frequencies between which the time delay is being calculated. As mentioned above, DM is the dispersion measure, defined to be the integrated column density of free electrons along the line of sight, i.e.,

$$
D M=\int_{0}^{d} n_{\mathrm{e}} d l
$$

Here $n_{\mathrm{e}}$ is the electron number density, $d$ is the distance to the pulsar and $d l$ is the infinitesimal path length along the line of sight. For terrestrial sources, $n_{\mathrm{e}}$ is negligible, so RFI will have a DM of $0 \mathrm{pc} \mathrm{cm}^{-3}$.

\subsubsection{Dedispersion}

Since the time delay caused by the ISM is quadratic, the recorded signal should show a quadratic sweep from high to low frequencies if it is astrophysical in nature. However, this dispersion decreases the strength of the signal from the pulsar, making it difficult to detect. In order to correct for this dispersion, a process known as dedispersion is carried out. This involves shifting the frequency channels in the data to remove the quadratic sweep. The amount the channels are shifted is determined by the DM being corrected for. In a search, however, the DM of a pulsar is not previously known. Therefore, a search over many DMs is necessary in order to find new pulsars.

Searching over too many DMs can be costly in terms of computing power, though. On the other hand, coarse sampling can lead to a loss in sensitivity to any pulsar with a DM between two consecutive trial DMs. Therefore, it is necessary to 
choose an optimal value for the DM step, so that a range of DMs can be reliably covered by as few steps as possible. If a time series containing pulsar data is dedispersed at a DM that differs from the true DM by $\Delta \mathrm{DM}$, the effective width of the resulting pulse, as given by Lorimer \& Kramer (2004), will be

$$
W_{\text {eff }}=\sqrt{W_{\text {int }}^{2}+\left(8.3 \times 10^{6} \mathrm{~ms} \times|\Delta D M| \times \Delta f / f^{3}\right)^{2}},
$$

where $W_{\text {int }}$ is the intrinsic pulse width, $\Delta \mathrm{f}$ is the bandwidth in $\mathrm{MHz}$, and $\mathrm{f}$ is the center frequency in MHz. This increased pulse width will decrease the signal-to-noise ratio $(\mathrm{S} / \mathrm{N})$ of the pulse (Lorimer \& Kramer, 2004), given by

$$
S / N \propto \sqrt{\frac{P-W_{\mathrm{eff}}}{W_{\mathrm{eff}}}} .
$$

Here, $P$ is the spin period of the pulsar. Ideally, the smallest effective width that can be obtained is constrained by the sampling time. Therefore, a good choice of DM step is one in which the delay between the highest and lowest frequency is equal to the sampling time. Then, following Lorimer \& Kramer (2004), the $i^{\text {th }}$ value of the DM becomes

$$
D M_{\mathrm{i}}=1.205 \times 10^{-7} \mathrm{~cm}^{-3} \mathrm{pc}(i-1) t_{\mathrm{samp}}\left(f^{3} / \Delta f\right),
$$

where $t_{\text {samp }}$ is the sampling time, and $f$ and $\Delta f$ are again in MHz. 


\subsubsection{Frequency Domain Searches}

While the first pulsar was discovered through visual detection of its single pulses (Hewish et al., 1968), similar to the way RRATs are discovered, most pulsars are not strong enough to be detected this way. However, since we know the pulses are periodic, we can make use of the Fast Fourier Transform (FFT) in order to to detect the periodicity of the pulsar in the frequency domain. First, the raw data are dedispersed at many different trial DMs. The frequency channels of each sample are added together, forming a profile of amplitude as a function of time. These resulting time series are then transformed via FFT. This leads to a 'power spectrum,' a plot of the power of each Fourier component as a function of frequency. Any periodic signal will produce a spike in this spectrum. Pulsars are therefore easily detected by setting a threshold and recording the frequency of any feature that exceeds that threshold.

A spike in the power spectrum, described above, represents a sinusoidal signal with a period represented by the location of that spike. A signal from a real pulsar, however, is not sinusoidal. Instead, the pulsar has some duty cycle, defined as the pulse width divided by the period, which is only a few percent. The FFT of a narrow pulse such as this produces not one but many spikes in the power spectrum. The first spike, the fundamental frequency, is followed by a number of harmonics, spikes at frequencies that are factors of two higher than the fundamental. The power from the pulsar is spread throughout these harmonics, decreasing the strength at the fundamental frequency. To recover the power spread over the harmonics, they 
are summed several times. Although the noise grows with each summation by a factor of $\sqrt{2}$, the powers of the harmonics add directly, resulting in a net gain in the resulting signal by $\sqrt{2}$.

\subsubsection{Acceleration Searches}

The above method works well for solitary pulsars, but we know that many pulsars (especially MSPs) are found in binary systems. The motion of the pulsar around its companion will cause the apparent period to be Doppler shifted. The result of this is that the power in Fourier space will be spread over many bins, significantly decreasing the strength of the spectral feature. If the orbital parameters of the system are known, it is straightforward to correct the time series based on the known radial velocity of the pulsar along the line of sight. However, if the orbital parameters are unknown, e.g., in pulsar searches, modeling the radial velocity is too computationally intensive. Instead, we assume that the pulsar experiences a constant acceleration throughout its orbit, so that the line-of-sight velocity component is

$$
V_{1}(t)=a_{1} t
$$

where $a_{1}$ is the line-of-sight component of the acceleration of the pulsar and $t$ is time. Then searching over different values of radial velocity simplifies to searching over a range of accelerations. Similar to dedispersion, searching over many accelerations requires computing an optimal acceleration step size. This is accomplished by determining the number of Fourier bins that an accelerated signal will occupy if 
no correction is made. Given an observation of length $T$, this is given by

$$
N_{\text {drift }}=a_{1} \nu_{0} T^{2} / c
$$

where $\nu_{0}$ is the true spin frequency of the pulsar. The optimal step size, $\Delta a_{1}$, should then ensure that $\Delta N_{\text {drift }}<1$, meaning $\Delta a_{1}<c P / T^{2}$, where $P$ is the spin period of the pulsar.

The above method works well for shorter data sets, but the need to FFT the data after each acceleration trial makes it computationally intensive for longer data sets. An efficient solution to this problem is to perform the correction in the frequency domain. There are a number of ways in which this correction can be made which we outline below:

- Frequency domain acceleration searches As stated above, an accelerated signal will have its power spread over several Fourier bins. This same behavior can be seen by convolving a stationary signal with a finite impulse response filter. Therefore, applying an inverse finite impulse response filter to accelerated data will reconstruct the power into a single bin (Ransom et al., 2001). While an array of inverse filters need to be tested, this method is less computationally intensive than the acceleration search described above.

- Stack-slide searches For extremely large searches, the above acceleration search becomes inefficient. A much quicker implementation is to break the observation into a series of smaller data sets. This effectively reduces the 
observation length $T$ in Equation 1.9, which in turn increases the width of each Fourier bin, so that a signal can drift more before leaking into another bin. Also, the shorter observation time allows less time for a signal to drift (Faulkner et al., 2004).

- Phase-modulation searches For pulsars with orbital periods much less than the integration time, the motion of the pulsar can no longer be modeled by Equation 1.8. The result of observing several orbits of a pulsar will be a range of frequencies covered in the Fourier domain. Fourier transforming this range of frequencies will result in the detection of the orbital period (Jouteux et al., 2002; Ransom et al., 2003).

- Dynamic power spectrum searches The above searches are optimized both for pulsars in binary orbits which are much longer and much shorter than the observation time. For systems which have orbital periods on the order of the observation length, the observation is split into smaller data sets as above. Each set of data is Fourier transformed and summed harmonically. A resulting frequency versus time plot known as a dynamic power spectrum can then be viewed by eye to look for the sinusoidal signature of an accelerated pulsar (Lyne et al., 2000).

\subsubsection{Candidate Selection}

Top periods and DMs returned by these searches are stored as candidates. These candidate periods and DMs are then used to dedisperse and fold the raw 
data, producing diagnostic plots which can then be viewed to confirm the validity of a candidate. In the folding process, which is required to obtain the integrated pulse profiles to produce these plots, an array of equally spaced bins spanning the pulse period is created. Then, for each sample in a dedispersed time series, a phase relative to the pulse period is calculated. That sample is then added to the bin which best matches its phase.

An example set of diagnostic plots is shown in Figure 1.5, which is a detection of PSR J1327-6301. While some of the subplots are more useful than others in confirming if a candidate is real, for most pulsars all of them must be considered together to make a determination. The most important subplots are the integrated pulse profile (upper left), pulse phase versus observation time (lower left), pulse phase versus frequency (middle), and DM versus $\chi^{2}$ (bottom middle). Here, $\chi^{2}$ is a measure of departure from random noise, with a higher value denoting a greater significance. It is defined as

$$
\chi^{2}=\frac{1}{\sigma_{\mathrm{p}}^{2}} \sum_{i=1}^{n_{\text {bins }}}\left(p_{\mathrm{i}}-\bar{p}\right)^{2}
$$

where $\bar{p}$ and $\sigma_{\mathrm{p}}^{2}$ are the mean and variance of pure Gaussian noise, and $p_{\mathrm{i}}$ is the amplitude of the $i^{\text {th }}$ bin of a folded profile. Often, instead of $\chi^{2}$, the reduced $\chi^{2}$ statistic, $\chi_{\mathrm{r}}^{2}$, is used. It is simply the value of $\chi^{2}$ divided by the number of degrees of freedom. For pure Gaussian noise, the ideal value for $\chi_{\mathrm{r}}^{2}$ is around 1. Most pulsar candidates begin to become discernible above the noise once $\chi_{\mathrm{r}}^{2}>2$.

In practice, judgment on whether to follow up pulsar candidates is made based 

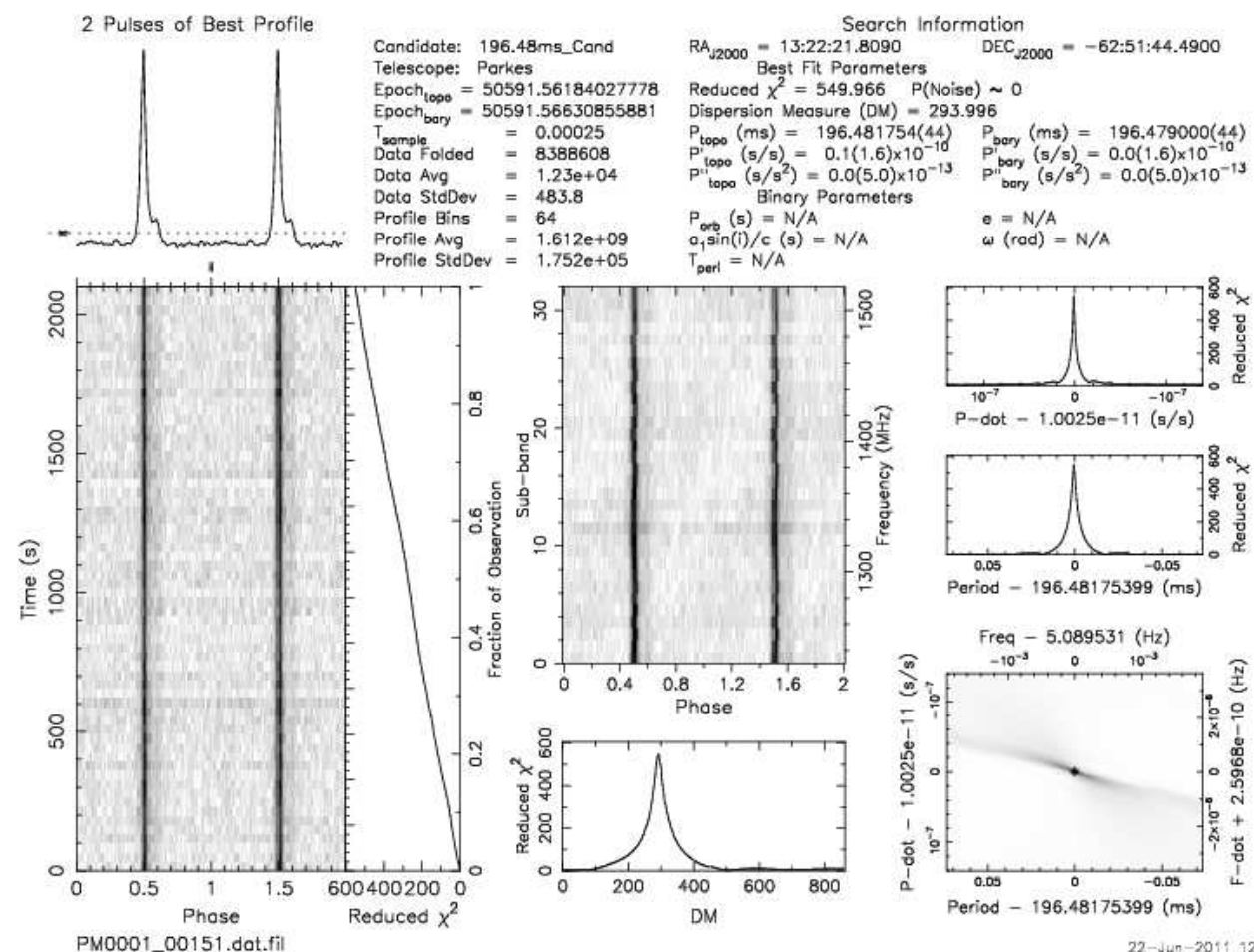
$P_{o r b}(s)=N / A$ $\mathrm{o}_{1} \sin (\mathrm{i}) / \mathrm{c} /(\mathrm{s})$ $\omega(\mathrm{rod})=N / A$
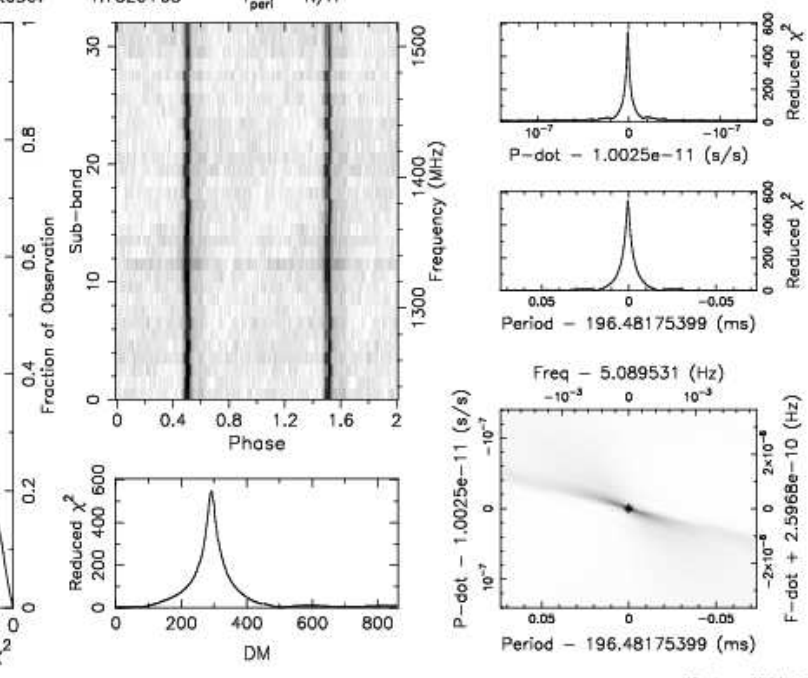

Figure 1.5: Example of a diagnostic plot output by our periodicity searches.

on the inspection of all of the diagnostics shown in Figure 1.5. Unfortunately, due to RFI and other false detections, candidates can number in the thousands or even hundreds of thousands. Therefore, either enormous manpower is needed to view the resulting plots, or restrictions need to be placed on certain parameters of the candidates in order to decrease the number of candidates to be viewed. These constraints can be placed on the period, DM, or the signal-to-noise $(\mathrm{S} / \mathrm{N})$ of the candidate.

\subsection{Pulsar Timing}

We have mentioned to this point that pulsars behave reliably, having known periods and period derivatives which allow us to predict, with great accuracy, when 
we should see pulses. While search output gives a good approximation of many of the parameters associated with a pulsar, the incredibly accurate measurements are the result of pulsar timing. Pulsar timing aims to account for every rotation of a pulsar by measuring the phase of the pulse, and the resulting timing solution is a list of the pulsars spin, astrometric, and binary parameters (if present). The pulse phase can be described by a Taylor expansion as

$$
\phi(t)=\phi_{0}+\frac{1}{2 \pi} \nu_{0}\left(t-t_{0}\right)+\frac{1}{4 \pi} \dot{\nu_{0}}\left(t-t_{0}\right)^{2}+\ldots
$$

where $\phi_{0}$ is the phase at time $t_{0}, \nu_{0}$ is the initial spin frequency, and $\dot{\nu}_{0}$ is the time derivative of $\nu_{0}$.

Timing observations are normally carried out over an extended period of time, with increasing duration between observations. The initial observations should be close, in order to avoid pulse numbering ambiguities that may arise from poorly known initial model parameters. For each observation, a time of arrival (TOA) is calculated. A TOA is defined as the arrival time of the pulse closest to the middle of an observation. Since most pulses span multiple time samples, it is important to designate one part of the pulse to be used to calculate each TOA. This is accomplished by creating a template. Since we know that the average profile of most pulsars is stable, we can use a high $\mathrm{S} / \mathrm{N}$ folded profile for this purpose. To remove any effects of noise from the template, it is typically fit with Gaussians. This Gaussian template and the observed profile are then Fourier transformed before being cross-correlated. Through $\chi^{2}$-minimization, a time shift between the template and 
profile can be measured, which is then used to calculate a TOA.

Given multiple TOAs, the parameters are then fit, through least-squares fitting, to these TOAs. Once the initial parameters, like period, have been accurately determined, observations can become less frequent. Observations over an extended period of time are still required, as errors in some parameters, like period derivative and position, will only become apparent over longer timescales (see Figure 1.6). Thus, it is important to calculate the initial values as accurately as possible, so that parameters that cause changes over longer periods of time can be better fit. Once a timing solution has been determined, post-fit residuals, the differences between the model predictions and the actual TOAs, should show a Gaussian distribution around zero, and the root mean square (RMS) of the distribution should ideally be on the order of the uncertainties in the TOAs.

As it turns out, the best timing solutions are acquired for MSPs. There are a few reasons for this. First, the uncertainty in a TOA measurement is given by

$$
\sigma_{\mathrm{TOA}} \simeq \frac{W}{S / N}
$$

Therefore, millisecond pulsars, with their short periods and narrow pulses, will allow TOAs to be measured more precisely than for a normal pulsar. Secondly, their short periods allow many pulses to be recorded in a short period of time, leading to very stable pulse profiles for use as templates. Finally, their small period derivatives lead to highly stable rotation, which makes finding a timing solution which remains accurate over long periods of time much easier than for normal pulsars. 

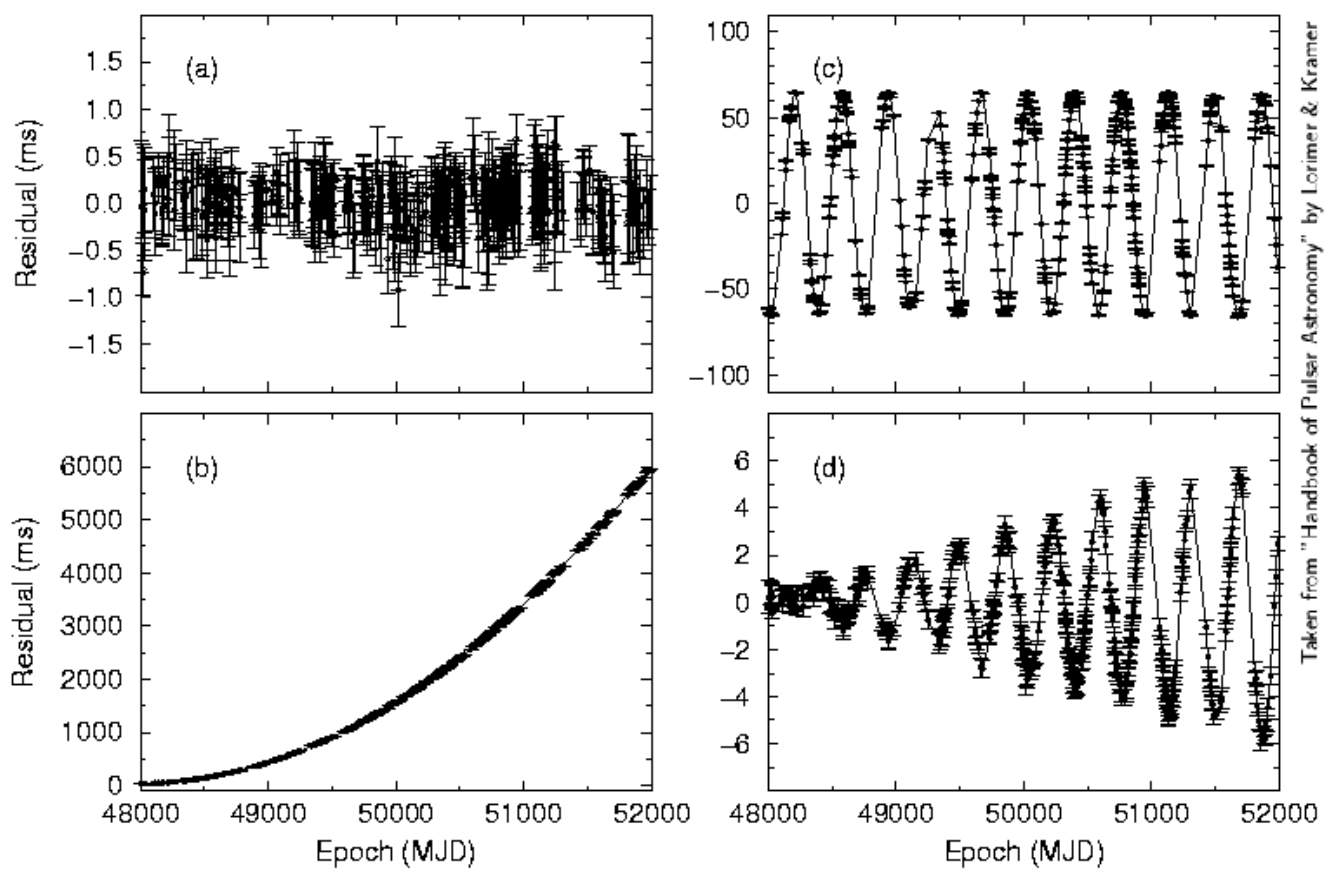

Figure 1.6: (a) Timing residuals for a correct timing solution showing a random distribution around a mean of zero. (b) Systematic increase in residuals due to an improper value of $\dot{P}$. (c) Variation in residuals due to an incorrect position. The sinusoidal nature, with a period of one year, is due to the motion of the Earth around the Sun. (d) Residuals due to neglecting the pulsar's proper motion. In each of these cases, the difference of the incorrect parameter from its true value is small. Figure used with permission from the Handbook of Pulsar Astronomy (Lorimer \& Kramer, 2004). 
The accuracy of pulsar timing solutions can be utilized in a number of ways. The most notable result of pulsar timing so far has been the confirmation of gravitational radiation from the observed shift in the periastron passage of the binary pulsar B1913+16 (Taylor \& Weisberg, 1989). This effect results from the orbit of these two neutron stars shrinking by as little as $1 \mathrm{~cm} \mathrm{day}^{-1}$. Another noteworthy accomplishment of pulsar timing was the timing of the first double pulsar system, J0737-3039 (Burgay et al., 2003; Lyne et al., 2004). The orbital period of this system is roughly 2.4 hours, resulting in a compact, relativistic orbit which allows for the measurement of relativistic Keplerian orbital parameters. The measurement of these parameters not only test the validity of theories of strong-field gravity, they also constrain the masses of the pulsars in this system. Accurate mass calculations for pulsars are important, as they can help to determine the neutron star equation of state. Demorest et al. (2010) originally measured the Shapiro delay ${ }^{4}$ in the binary system containing PSR J1614-2230. Using this, they were able to infer a mass for the pulsar of $\sim 2 \mathrm{M}_{\odot}$, which ruled out many of the proposed equations of state (Schwarzschild, 2011). Presently, pulsar timing is often used to time and monitor a large array of MSPs to extreme accuracy. Since MSPs generally haven timing solutions with lower RMS post-fit residuals than solutions for normal pulsars, the RMS residuals for some MSPs can be as low as 100 ns (van Straten et al., 2001). This well-timed group of MSPs, called a pulsar timing array, is now being actively used to search for low-frequency gravitational waves (Jenet et al., 2009; Ferdman

\footnotetext{
${ }^{4}$ The Shapiro delay is the increase in light travel time due to a signal propagating through curved space-time near a massive body.
} 
et al., 2010; Hobbs et al., 2010; Manchester et al., 2013).

\subsection{Outline of Thesis}

The goals of the work presented in this thesis are to determine the emission mechanisms present in pulsars through studies of giant pulses and single pulses, and to discover and characterize new pulsars. Chapter 2 shows the results of a long-term study of giant pulses from the Crab pulsar. This study was performed in order to constrain the emission mechanism of giant pulses. Specifically, it tested a theory that giant pulses are the result of increased pair production in the pulsar magnetosphere. The radio observations, data reduction of both radio and $\gamma$-ray data, and giant pulse properties are discussed. Chapter 3 presents the discovery of six new pulsars found in archival data, as well as timing solutions for two of the pulsars. Chapter 4 details the search for highly-dispersed radio bursts out to large distances, as well as the study and classification of single pulses from pulsars and RRATs. General conclusions of this work are given in Chapter 5. 


\section{Chapter 2}

\section{A Giant Sample of Giant Pulses from the Crab Pulsar}

This chapter was originally published as a refereed paper in the Astrophysical Journal by M. B. Mickaliger et al. in November 2012. Minor word changes from the original have been made where appropriate.

\subsection{Introduction}

The Crab pulsar was discovered by Staelin \& Reifenstein in 1968 through its giant pulses. Giant pulses (GPs) can be thousands of times brighter than the average pulse. The temporal occurrence of GPs is random but at frequencies below $3 \mathrm{GHz}$ they always occur at the phase of either the Crab pulsar's main pulse (MP) or interpulse (IP) (Lundgren, 1994). At frequencies from $4 \mathrm{GHz}$ to $8.4 \mathrm{GHz}$, GPs are emitted at the phases of the MP and IP as well as at the phases of two additional high-frequency components (Moffett \& Hankins, 1996). Above 8.5 GHz, GPs are again seen at only the phases of the MP and IP (Jessner et al., 2010)

The emission mechanism of GPs is still an open question (e.g. Petrova, 2006; Istomin, 2004; Weatherall, 1998). GPs could be caused by increased pair production in the magnetosphere, increased coherence of synchrotron emission, or changes in beaming direction. Correlating radio GPs with high-energy photons is one way to

determine if increased pair production is a major cause of GPs. A recent model 
(Lyutikov, 2007) proposes that GPs are generated on the last closed magnetic field line near the light cylinder via anomalous cyclotron resonance. If this is true, there would be an increase in $\gamma$-rays at the times of radio GPs. Anomalous cyclotron resonances have been previously proposed as a cause of GP emission by Lyutikov et al. (1999) and Machabeli \& Usov (1979). We expect the $\gamma$-rays to be phase aligned with the radio GPs at $1.2 \mathrm{GHz}$ as the $\gamma$-ray and radio profiles are aligned (see Figure 2.1). Since we have such a large data set of GPs, we can correlate them with $\gamma$-ray photons to test this model. Correlations between radio GPs and $\gamma$-ray photons have been carried out previously by Bilous et al. (2011) and Lundgren et al. (1995). Shearer et al. (2003) correlated radio GPs and optical photons and found a slight correlation, and work by Collins et al. (2012) supports this result.

The Fermi Large Area Telescope (LAT) is a pair conversion telescope that operates in the energy range from $20 \mathrm{MeV}$ to $300 \mathrm{GeV}$. It has a large field of view $(2.4 \mathrm{sr})$, which allows it to rapidly map the entire sky, and very good angular resolution, minimizing background contamination (Atwood et al., 2009). The Crab pulsar is a bright Fermi source and has been studied at these energies. Abdo et al. (2010) found that the $\gamma$-ray profile is double peaked and matches the $1.4 \mathrm{GHz}$ profile, with the $\gamma$-ray peaks leading the radio peaks by $\sim 0.01$ phase. Since the LAT covers the energies predicted by Lyutikov (2007) and observes the Crab pulsar multiple times per day, we can use Fermi data to test Lyutikov's theory.

The power-law nature of the amplitude distribution of GPs is well known (e.g. Argyle \& Gower, 1972; Popov \& Stappers, 2007), but there have been varying values for the power-law index calculated, even for similar frequencies (e.g. Karuppusamy 
et al., 2010; Bhat et al., 2008). With our large sample of GPs, we can calculate power-law indices over a long timespan. Since the amplitude distribution of pulses from many pulsars is lognormal (Ritchings, 1976), GPs must have a different emission mechanism. By comparing our power-law index with those of other neutron star source classes (pulsars, magnetars, RRATs), we may be able to put constraints on the GP emission mechanism.

A $\gamma$-ray flare was recently observed from the Crab Nebula by the AGILE satellite (Tavani et al., 2011). The flare lasted from MJDs 55457-55461 and was also observed by Fermi (Hays et al., 2010). This occurred during the span of our observations, so we can compare radio and $\gamma$-ray properties of the pulsar from before the flare with a few days of data taken about two months after the flare to verify that the flare was not associated with the pulsar. Current theories suggest that the flare was caused by electrons accelerated by magnetic reconnection at the termination shock of the Crab Nebula (e.g. Bednarek \& Idec, 2011; Bykov et al., 2012; Cerutti et al., 2012).

The plan for the rest of this Chapter is as follows. Section 2.2 describes the radio observations and outlines the radio analysis. Section 2.3 discusses the GP statistics and the power-law index calculations. Section 2.4 details the correlation between 43-m and GBT GPs. Section 2.5 presents the Fermi data used and its reduction as well as the $43-\mathrm{m} /$ Fermi correlation. Section 2.6 discusses the recent $\gamma$-ray flare. Finally, conclusions are offered in Section 2.7 . 


\subsection{Radio Observations}

We collected data over the course of 15 months using the 43-m telescope (hereafter GB43), located at the National Radio Astronomy Observatory site in Green Bank, WV. The GB43 was funded by MIT Lincoln Labs to perform bistatic radar observations of the ionosphere (Langston, 2007). Most of the observations had a center frequency of $1.2 \mathrm{GHz}$ with a usable bandwidth of $400 \mathrm{MHz}$, with a handful of the later observations centered at $330 \mathrm{MHz}$, with a usable bandwidth of 150 MHz. All of the observations were taken using 4096 frequency channels and varying sampling times, listed in Table 2.1. Some of the sampling times used were rather long, and this was done to decrease the data volume. In total, we observed the Crab pulsar for 100 hours at $1.2 \mathrm{GHz}$ and seven hours at $330 \mathrm{MHz}$. The $330 \mathrm{MHz}$ mode was implemented later and used because pulsars, in general, are stronger at lower frequencies and exhibit a steep spectral index (Lorimer et al., 1995). Even though the smaller bandwidth at $330 \mathrm{MHz}$ reduces sensitivity by almost a factor of 2 , the steep spectrum of the Crab pulsar ( $\alpha=3.1 \pm 0.2$ (Lorimer et al., 1995), where $\mathrm{S}=\nu^{-\alpha}$ ) means that the pulsar is 55 times brighter at $330 \mathrm{MHz}$ than at $1.2 \mathrm{GHz}$. The flux density from the Crab Nebula is greater at lower frequencies (Cordes et al., 2004 ) but, due to a shallow power-law index $\left(\nu^{-0.27}\right)$ (Allen, 1973; Bietenholz et al., 1997), at $330 \mathrm{MHz}$ (flux density $\sim 1288 \mathrm{Jy}$ ) is only 1.4 times brighter than at 1.2 GHz (flux density 909 Jy). Therefore there should be many more GPs above our $10 \sigma$ threshold at $330 \mathrm{MHz}$ than at $1.2 \mathrm{GHz}$.

Data were taken with WUPPI, the West Virginia University Ultimate Pulsar 
Processing Instrument. WUPPI is a clone of GUPPI (Green Bank Ultimate Pulsar Processing Instrument) (DuPlain et al., 2008) for use with the GB43. GUPPI is a flexible digital backend for the GBT. Like GUPPI, WUPPI is built from reconfigurable off-the-shelf hardware and software available from the CASPER (Center for Astronomy Signal Processing and Electronics Research) group (Parsons et al., 2009). Both GUPPI and WUPPI sample the data with 8-bit precision over bandwidths as large as $800 \mathrm{MHz}$, and are capable of recording all four Stokes parameters. To ease disk space usage, only total intensity (Stokes I) was recorded.

The data were processed using a real-time data reduction pipeline on a 16 processor mini-cluster. The pipeline is comprised of a set of scripts, built from freely available analysis software ${ }^{1}$, that can reduce a file in real time. First, a file in PSRFITS format (Hotan et al., 2004) has its mean bandpass divided out. Frequency channels that have intensities above the resulting mean are flagged as containing radio frequency interference (RFI) and removed. Then the resulting file is dedispersed at both a dispersion measure (DM) of zero and the DM of the Crab, which is $\sim 56.8 \mathrm{pc} \mathrm{cm}^{-3}$; the exact values for each observation, obtained from the Jodrell Bank Crab Pulsar Monthly Ephemeris² (Lyne et al., 1993), are listed in Table 2.1. The DM of the Crab pulsar can vary on a monthly timescale by $\sim \pm 0.01 \mathrm{pc}$ $\mathrm{cm}^{-3}$. In order to keep processing in real-time we did not correct for this. To ease space requirements, raw data were not kept, so we could not later correct for this variation in our data. Errors in GP arrival times due to an incorrect DM on the

\footnotetext{
${ }^{1}$ http://sigproc.sourceforge.net

${ }^{2}$ http://www.jb.man.ac.uk/ pulsar/crab.html
} 
order of $0.01 \mathrm{pc} \mathrm{cm}^{-3}$ are $\sim 20 \mu \mathrm{s}$, which is less than the sampling time. Both resulting time series are then searched for single pulses with a peak signal-to-noise $(\mathrm{S} / \mathrm{N})$ above $10 \sigma$ using our single pulse search (based on the algorithm described by Cordes \& McLaughlin (2003)). Our $10 \sigma$ definition of a GP is somewhat arbitrary because there is no set threshold for a GP as the weakest GPs have yet to be observed. The current population of observed GPs accounts for no less than $50 \%$ of the pulsed emission at frequencies around 1.6 GHz, and the inclusion of the weakest GPs could bring that number up to $90 \%$ (Majid et al., 2011). A GP time-of-arrival (TOA) (measured by taking the arrival time of the peak of the pulse), the $\mathrm{S} / \mathrm{N}$ of that peak, and the pulse width, taken as the width of a best-fit top-hat function, are recorded for each pulse. The TOAs from each time series are compared and if a pulse detected at the DM of the Crab is within $0.1 \mathrm{~s}$ of a zero DM pulse, the S/N is checked for both pulses ${ }^{3}$. If the $\mathrm{S} / \mathrm{N}$ is higher in the zero DM pulse, the pulse is assumed to be due to RFI and removed. The pipeline outputs a profile for each GP and produces an average folded profile for the entire observation, a plot of GP arrival time vs pulse phase, and an average GP profile, made by summing all of the individual GP profiles.

Figure 2.2 shows the number of GPs detected versus pulse phase. It is worth noting that, although there were only four post-flare observing epochs, there are many more GPs post-flare than were recorded in all of the pre-flare data. This was due to a receiver upgrade which resulted in a large increase in sensitivity between our

\footnotetext{
${ }^{3}$ This is of the order of the frequency dependent arrival time delay across the band, and is used as it removes very nearly all RFI, while removing few real GPs.
} 
observations on MJD 55412 and MJD 55516. At frequencies below $5 \mathrm{GHz}$ (Cordes et al., 2004), most detected GPs come at the phase of the MP, with the rest at the IP phase. In our data, $\sim 87 \%$ of detected GPs were at the phase of the MP, while the other $\sim 13 \%$ were at the phase of the IP. Only $5 \%$ of GPs detected by Cordes et al. (2004) at $1.2 \mathrm{GHz}$ were at the phase of the IP, but Karuppusamy et al. (2010) found that $\sim 12 \%$ of GPs detected at $1.4 \mathrm{GHz}$ were IP GPs. For detected pulses that show up out of phase with the MP and IP, the frequency versus time plots are checked by eye to see if they show the proper quadratic frequency sweep for a DM of $56.8 \mathrm{pc} \mathrm{cm}^{-3}$. If they do not, those pulses are removed. In most cases, the RFI removal process mentioned above removes most of these false detections before they are checked by eye. We found no events at phases other than those of the MP or IP that showed the proper frequency sweep to be a real GP.

Most of the GPs we detected have a constant intensity across the entire band. Some of them, however, show variations in amplitude as a function of frequency, which have been seen before (e.g. Karuppusamy et al., 2010). We also see variations in amplitude between days, which are likely due to refractive interstellar scintillation (RISS), which can affect the strength of pulses on a timescale of days.

In order to determine if the time stamps for the GB43 are accurate, a short ( 10 min) observation of the Crab pulsar was made contemporaneously on MJD 55406 at similar frequencies with the GB43 and GBT. The GB43 used a center frequency of $330 \mathrm{MHz}$, while the GBT used $350 \mathrm{MHz}$. GPs were detected in each data set and their TOAs were compared. As can be seen in Figure 2.3, the pulse shapes of contemporaneous GPs are similar, and the TOAs, converted to infinite 
frequency at the solar system barycenter, have identical arrival times to within the instrumental resolution.

Figure 2.4 shows the average profile of the Crab pulsar at $1.2 \mathrm{GHz}$ and 330 MHz. The intensities are in arbitrary units. The MP and IP are visible at both frequencies. The increased width at low-frequency is due largely to DM smearing, which is on the order of $2.4 \mathrm{~ms}$ at the bottom of the band. The $1.2 \mathrm{GHz}$ profile exhibits a weak low-frequency component (LFC), about one-tenth of a pulse phase ahead of the MP (e.g. Moffett \& Hankins, 1996). At $330 \mathrm{MHz}$ there is a precursor (PR) to the MP (e.g. Rankin et al., 1970) which is broad enough at this frequency that it shows up as the first peak of the double-peaked MP (see Karuppusamy et al., 2012, for the frequency evolution of the PR), which is visible in the low-frequency GBT profile from MJD 55406 (Figure 2.1). Due to a much larger effective resolution, the MP in the GB43 profile is unresolved and appears to have a single peak. Table 2.2 lists the effective resolutions at the top and bottom of the band for both the 330 $\mathrm{MHz}$ and $1.2 \mathrm{GHz}$ GB43 observations as well as the GBT $350 \mathrm{MHz}$ observation.

The full-widths-at-half-maximum (FWHMs) of the MP and IP from the GB43 profiles are $\sim 305 \mu$ s and $\sim 360 \mu$ s at $1.2 \mathrm{GHz}$ and $\sim 3.2 \mathrm{~ms}$ and $\sim 1.5 \mathrm{~ms}$ at $330 \mathrm{MHz}$. At $330 \mathrm{MHz}$, the MP is wider than the IP due to its overlap with the PR (Rankin et al., 1970). The average GP profiles, however, are narrower than the average folded profiles at both frequencies (see Figure 2.5), with FWHMs of $\sim 140 \mu$ s for both the MP and IP at $1.2 \mathrm{GHz}$, and $\sim 1.1 \mathrm{~ms}$ at $330 \mathrm{MHz}$ for both the MP and IP. It has been suggested by Popov et al. (2006) that every pulse at the phase of the MP and IP is a GP, and that normal emission only comes from the PR, where 
no GPs have been seen. We also have not seen any GPs at the phase of the PR at $330 \mathrm{MHz}$ in our data. Even though our observed GP profiles are narrower than the average folded profiles, this hypothesis is not ruled out. Popov \& Stappers (2007) found that pulse width is inversely proportional to pulse intensity, so lowering our $\mathrm{S} / \mathrm{N}$ definition of a GP would include wider GPs which could possibly increase the width of the average GP profile to that of the folded profile. Also, the phases of weaker GPs may have more deviation from the center phase of the MP and IP than stronger GPs, leading to a wider average profile. Unfortunately, we were unable to lower our GP threshold below $10 \sigma$ as this resulted in many spurious pulses.

\subsection{Amplitude Distributions}

A total of 93698 GPs were observed with the GB43. At $1.2 \mathrm{GHz}$ we recorded 76707 GPs at the phase of the MP and 10871 GPs at the phase of the IP, and at $330 \mathrm{MHz} 5232 \mathrm{MP}$ and $888 \mathrm{IP}$ GPs were recorded. Due to low GP statistics on some days, only 78574 MP GPs and 9693 IP GPs were used in fitting the amplitude distributions.

We calculated, through least-squares fitting, power-law indices for the differential amplitude distributions of MP and IP GPs at both $1.2 \mathrm{GHz}$ and $330 \mathrm{MHz}$ for each day separately. Figure 2.6 is an example of a power-law fit for the MP. We noted that the power-law index of GPs varied daily, as was previously reported by Lundgren et al. (1995) at similar frequencies. This gave a range of power-law indices from $2.1 \pm 0.3$ to $3.1 \pm 0.2$ for MP GPs at $1.2 \mathrm{GHz}, 2.4 \pm 0.4$ to $2.81 \pm 0.03$ for 
IP GPs at $1.2 \mathrm{GHz}, 2.5 \pm 0.2$ to $2.95 \pm 0.09$ for MP GPs at $330 \mathrm{MHz}$, and $2.4 \pm 0.2$ to 3.1 \pm 0.2 for IP GPs at $330 \mathrm{MHz}$. Table 2.3 lists the power-law indices for each day. These indices agree with other published power-law indices, listed in Table 2.4. The range of indices is likely due to wider GPs having steeper spectra, as was seen by Popov et al. (2008). We did not have the time resolution to separate the GPs by width, as the widest GPs reported by Popov et al. (2008) were $64 \mu \mathrm{s}$, which is the shortest sampling time we used.

We were unable to properly calibrate the data due to lack of any off-source pointings and/or observations with a pulsed calibrator. There were also doubts as to the stability of the receiver over long timespans. We were able to do a rough calibration, however, by correcting for RISS, which affects the strength of all GPs by the same amount, so there should be no change to the power-law slope. Rickett \& Lyne (1990) found that the RISS timescale for the Crab pulsar scales as $\nu^{-2.2}$. Based on this, the timescales at $1.2 \mathrm{GHz}$ and $330 \mathrm{MHz}$ are 1.7 days and 30 days, respectively. A Lomb-Scargle analysis (Scargle, 1982) of the periodicity of GP arrival times yielded periodicities of 0.41 days at $330 \mathrm{MHz}$ and 0.99 days at $1.2 \mathrm{GHz}$. The one day periodicity of the GP arrival times in the $1.2 \mathrm{GHz}$ data is on the order of the RISS timescale at that frequency, but the same periodicities were also seen in the randomized data set, so they are not significant. We attempted to correct for RISS by scaling observations based on the brightness of their folded profiles. However, if GPs dominate the average folded profile, as was seen by Popov et al. (2006), any intrinsic variations in their strength will affect this correction. To determine if this was true, we removed the GPs from the average profile. We found that at 
1.2 $\mathrm{GHz}$ the amplitude of the average profile without GPs was $1 \%$ less than the average profile with the GPs, and at $330 \mathrm{MHz}$ was $2 \%$ less. We therefore concluded that GPs do not dominate the average profile. One possible reason why our results are the opposite of what was seen by Popov et al. (2006) is that they recorded all GPs above $5 \sigma$, while we were only able to record GPs above $10 \sigma$. Therefore, it is possible that weaker GPs dominate the average profile. If the average profile is truly dominated by GPs, then correcting for RISS is complicated, but since we do not see this, we used the following correction.

We took the brightest folded profile from each of our three observing epochs (pre-flare $1.2 \mathrm{GHz}, 330 \mathrm{MHz}$, post-flare $1.2 \mathrm{GHz}$ ) and scaled all of the folded profiles from those epochs to that profile. That provided us with a scaling factor for each day, by which we then multiplied the GP S/Ns. We also compared our indices with power-law indices of other source classes in an attempt to constrain the GP emission physics. Other source classes and their indices are listed in Table 2.5. Although most normal pulsars have lognormal distributions, some pulsars have amplitude distributions that have power-law tails. These power-law exponents are included in Table 2.5 under 'Normal Pulsars'. Our power-law indices match those of magnetars and RRATs, but do not match the power-law tails seen from normal pulsars.

Also seen in Figure 2.6 is a non power-law tail, which is seen on all days in both the MP and IP. This deviation is significant, and was also seen by Cordes et al. (2004), who postulated these outlying GPs could be supergiant pulses, indicating that there may be two distinct mechanisms for GP generation. These supergiant pulses account for slightly less than one percent of the GPs used in the amplitude 
distributions.

\subsection{Correlating Radio GPs from the GB43 and GBT}

For 16 hours over the span of eight days, we observed the Crab pulsar simultaneously with the GB43 and GBT. The center frequency for GB43 observations was $1.2 \mathrm{GHz}$ with $400 \mathrm{MHz}$ of usable bandwidth, while that of the GBT was 8.9 $\mathrm{GHz}$ with $800 \mathrm{MHz}$ of usable bandwidth. At high frequencies the majority of GPs come at the phase of the IP, with fewer MP GPs and few GPs from high-frequency components (HFCs) (see Figure 2.7 for a comparison of the folded profile at high and low frequencies). For this work, we did not use any HFC GPs in our correlation analysis. We matched the barycentered arrival times of the 39900 GPs with S/N $>10$ recorded with the GBT (1035 MP GPs, 38865 IP GPs) with the 7933 GPs recorded simultaneously with the GB43 (7466 MP GPs, 467 IP GPs). We found that 236 low-frequency MP GPs were also detected simultaneously at $8.9 \mathrm{GHz}$. These GPs were neither the strongest nor the weakest pulses from either data set. The chance probability of this occurring is zero percent. This is not surprising, as we know that the high- and low-frequency MP are the same component. We did expect, however, that all of the MP GPs detected at $8.9 \mathrm{GHz}$ would be the strongest of the MP GPs detected at $1.2 \mathrm{GHz}$, since the MP is significantly weaker at 8.9 $\mathrm{GHz}$ than at $1.2 \mathrm{GHz}$. All chance probabilities were calculated assuming a Poisson

distribution, using the formula $P=\frac{e^{-\lambda} \times \lambda^{K}}{K !}$, where $\lambda$ is the number of detections expected and $K$ is the number of detections actually recorded. 
Moffett \& Hankins (1996) first noticed that the low- and high-frequency IP components of the folded profile are separated by $10^{\circ}$, which is about $970 \mu \mathrm{s}$. We checked to see if any low-frequency IP GPs were simultaneously detected at 8.9 GHz. Only 23 were found ( $\sim 5 \%)$, with a $\sim$ zero percent chance occurrence, but this is expected since the spectral index of GPs is steep (Popov et al., 2008). We then checked to see if low- and high-frequency IP GPs commonly occurred within the same rotation period of the pulsar, as was previously seen by Popov et al. (2008). In our data, we found 15 instances $(\sim 4 \%)$ of low-frequency IP GPs occurring within one spin period of a high-frequency IP GP. The probability of this measurement happening by chance is $9 \%$. In four of these instances the GPs are within one $\mu \mathrm{s}$ of each other and the chance probability of this occurring is $\sim$ zero. In the other 11 instances, the GPs are almost one spin period apart. These occurrences are likely statistical, as there is a $10 \%$ chance of this occurring randomly. The fact that only four of the 1.2 GHz IP GPs occur within one $\mu$ s of an $8.9 \mathrm{GHz}$ IP GP suggests that the high and low-frequency IP may be created by different physical processes (Moffett, 1997) possibly due to emission from different regions in the magnetosphere (Hankins \& Eilek, 2007). However, since the chance probability of detecting four low- and high-frequency IP GPs within one $\mu \mathrm{s}$ is $\sim$ zero, it seems likely that the high-frequency IP is related to the low-frequency IP, as seen by Popov et al. (2008) in previous correlations of IP GPs at 600 and $4850 \mathrm{MHz}$. One possibility is that both the high and low-frequency IP are reflections of the MP off of the magnetosphere (Petrova, 2009). In this scenario, radio emission from the MP originating deep in the magnetosphere propagates through the electron-positron plasma that fills the 
magnetosphere. Transverse scattering causes the MP emission to be backscattered, causing it to arrive at a different pulse phase. If this was the case, we would expect a linear relationship between the strength of the MP and IP in the folded profile for each observation. As shown in Figure 2.8, we do see such a relationship at both 1.2 $\mathrm{GHz}$ and $330 \mathrm{MHz}$. The slopes for the $1.2 \mathrm{GHz}$ and $330 \mathrm{MHz}$ relations are $0.310 \pm 0.004$ and $0.58 \pm 0.08$, respectively.

\subsection{Correlating Radio GPs from the GB43 with $\gamma$-ray Photons from Fermi}

One of the predictions of Lyutikov (2007) is that there would be increased $\gamma$-ray flux during a GP. In order for this model to accurately reproduce the data, the plasma density of the GP emission region must be $\sim 10^{5}$ higher than the minimum Goldreich-Julian density and the duty cycle of the pulsar must be 0.001. This increase in density could be due to enhanced pair production in the pulsar magnetosphere. If correct, these high-energy particles, produced during reconnection close to the Y point, where the last closed magnetic field lines approach the light cylinder at the magnetic equator (Lyutikov, 2007), are expected to produce curvature radiation from $0.1-100 \mathrm{GeV}$ (depending on the value of the Lorentz factor $\gamma$ ) at the time of a GP. This curvature radiation would cause an increase in $\gamma$-rays at the times of radio GPs.

Data were downloaded from the Fermi online archive $^{4}$ for days when radio

\footnotetext{
${ }^{4}$ http://fermi.gsfc.nasa.gov/cgi-bin/ssc/LAT/LATDataQuery.cgi
} 
observations occurred. 'Source' class events from Pass 7 data above $100 \mathrm{MeV}$ were selected in an energy dependent radius $\left(\theta<\operatorname{Max}(6.68-1.76 \log (\mathrm{E}), 1.3)^{\circ}\right.$, where $\mathrm{E}$ is the energy of the photon in $\mathrm{MeV}$ ) around the position of the pulsar (Abdo et al., 2010). Only photons in Good Time Intervals (GTIs) were selected and those with a zenith angle $>100^{\circ}$ were excluded to discriminate against $\gamma$-rays generated in the Earth's atmosphere. The photons were converted to infinite frequency at the solar system barycenter using the gtbary utility from the Fermi Science Tools package.

We then searched for coincidences between radio GPs and $\gamma$-ray photons. Due to clock errors in the backend on four days at $1.2 \mathrm{GHz}(55097,55257,55290,55346)$, only $75131 \mathrm{MP}$ and $10771 \mathrm{IP}$ GPs were used. However, all of the $5232 \mathrm{MP}$ and 888 IP GPs recorded at $330 \mathrm{MHz}$ were used. This resulted in a total of $92022 \mathrm{GPs}$ and $393 \gamma$-ray photons, with an average $\gamma$-ray photon rate of $\sim 16$ photons/hour, in agreement with the 15 photons/hour seen by Abdo et al. (2010). Fermi observes the Crab pulsar for about 11 hours per day, resulting in about 34 hours of simultaneous observing time. We also made energy cuts and searched for coincidences between radio GPs and $\gamma$-ray photons above both $500 \mathrm{MeV}$ and $1 \mathrm{GeV}$.

Based on comparisons with correlations of randomized data, we found no significant correlation between MP or IP GPs at both $1.2 \mathrm{GHz}$ and $330 \mathrm{MHz}$ and $\gamma$-ray photons out to a time lag of $\pm 3 \times 10^{6}$ spin periods. Since there was not necessarily the same number of randomized $\gamma$-ray photons during an observation as there were real $\gamma$-ray photons, we used this large time lag to make sure that there were the same number of real and randomized correlations once all of the $\gamma$-ray photons were included. The mean and standard deviation of the randomized correlations were 
calculated by randomly assigning $\gamma$-ray photon arrival times within GTIs and correlating them with GP TOAs. Figure 2.9 shows the results of this correlation analysis. The random correlations are the mean of 10000 trials, and the error bars represent one standard deviation. The largest deviation from the mean is a $2.4 \sigma$ negative correlation between $\gamma$-ray photons and post-flare $1.2 \mathrm{GHz}$ MP GPs at a time lag of 20000 spin periods, while the largest correlation is $2.1 \sigma$ for the pre-flare IP at $1.2 \mathrm{GHz}$ at a time lag of 200000 spin periods. The maximum correlation/negative correlation for each frequency at each energy cut is shown in Table 2.6. For both the MP and IP for each of the three data sets (pre-flare $1.2 \mathrm{GHz}, 330 \mathrm{MHz}$, post-flare $1.2 \mathrm{GHz}$ ), there are at most two time lags where the correlation exceeds $2 \sigma$. There are a total of 49 time lags included in the correlation, so two time lags are only four percent of the total data, while we statistically expect five percent of the data to have correlations beyond $2 \sigma$. Given the low significance and large time lag of these results, they do not provide any compelling case for a physical origin.

Selecting only $\gamma$-ray photons above $500 \mathrm{MeV}$ resulted in a total of 119 photons. The maximum correlation for this more restricted set was $2.3 \sigma$ at a time lag of one spin period for pre-flare MP at $1.2 \mathrm{GHz}$. The maximum negative correlation was $3.5 \sigma$ at a time lag of 20000 spin periods for the post-flare MP at $1.2 \mathrm{GHz}$. No more than one time lag for any data set had a correlation over $2 \sigma$, so those measurements above $2 \sigma$ are statistically insignificant. Statistically, less than half of a percent of the data should be greater than $|3 \sigma|$, and two percent of our data exceed $3.5 \sigma$. However, this is likely due to the coarseness of our time lags. Given more time lags, we would expect less than half a percent of our data to exceed $3 \sigma$. 
Only $65 \gamma$-ray photons had energies above $1 \mathrm{GeV}$. The maximum correlation occurred in the $1.2 \mathrm{GHz}$ pre-flare $\mathrm{MP}$ at $3.2 \sigma$ and a time lag of one spin period. The maximum negative correlation was $1.2 \sigma$, which occurred in the $1.2 \mathrm{GHz}$ pre-flare IP at a time lag of 200 spin periods. As with the $500 \mathrm{MeV}$ energy cut, we only see at most one time lag above $2 \sigma$, which is insignificant. Similarly, the $3.2 \sigma$ correlation is likely due to the coarseness of our time lags, and would likely drop given more time lags.

\subsection{Crab Nebula $\gamma$-ray Flare}

The recent $\gamma$-ray flare from the Crab Nebula was detected by the AGILE satellite above $100 \mathrm{MeV}$ (Tavani et al., 2011). Elevated $\gamma$-ray flux was observed from MJDs 55457-55461. No variations in pulse shape were found at $\gamma$-ray (Hays et al., 2010), X-ray (Tavani et al., 2011), or radio (Espinoza et al., 2010) energies. Espinoza et al. (2010) also found no increase in pulsed radio flux, glitches, or changes in DM around the date of the flare. Our closest observations before the flare were on MJD 55412 at $330 \mathrm{MHz}$ and MJD 55352 at $1.2 \mathrm{GHz}$, and our first observation after the flare was on MJD 55516 at $1.2 \mathrm{GHz}$.

We looked for changes in the average pulse profile, GP shape, power-law index, and $\gamma$-ray correlation in our pre- and post-flare $1.2 \mathrm{GHz}$ data. We found no significant differences in the pulse profile shape and the average GP shape (Figure 2.10) before and on four days about two months after the flare. The pre-flare MP power-law indices are in the range $2.1 \pm 0.3$ to $3.1 \pm 0.2$, while the post-flare power-law 
indices for the MP and IP are 2.56 \pm 0.05 to $2.93 \pm 0.05$ and $2.4 \pm 0.4$ to $2.81 \pm 0.03$, respectively. We were unable to calculate power-law indices for the IP pre-flare due to too few IP GPs. The largest negative correlation between $\gamma$-ray photons and GPs was seen in the post-flare data, but it is still on the order of correlations/negative correlations seen in pre-flare data, both at $1.2 \mathrm{GHz}$ and $330 \mathrm{MHz}$. Therefore, the flare seems to be unassociated with the pulsar, and was likely caused by magnetic reconnection.

\subsection{Conclusions}

We compared GB43 and GBT GPs and found that 3\% of MP GPs and 5\% of IP GPs at $1.2 \mathrm{GHz}$ were simultaneously detected at $8.9 \mathrm{GHz}$. The probability of either of these events occurring by chance is $\sim$ zero. Also, an additional four IP GPs at $1.2 \mathrm{GHz}$ were within one $\mu$ s of an IP GP at $8.9 \mathrm{GHz}$, with a $\sim$ zero percent chance probability. This may suggest that, although the folded profiles are much different at the two frequencies, the emission mechanism is similar. However, the low percentage of low- and high- frequency IP GPs within one $\mu$ s could mean that the IP emission mechanism is different at higher frequencies, especially since the high-frequency IP is shifted by 10 degrees.

Long observations allowed us to collect the largest sample of GPs to date, which we then used to calculate power-law indices for fits to amplitude distributions. These power-law indices agree with previously published values. A comparison of these indices to other source classes shows that GP emission is not exactly related 
to emission from other source classes and most closely matches the emission from magnetars and RRATs.

We found no significant correlations between GB43 GPs and Fermi $\gamma$-ray photons in the energy range $0.1-100 \mathrm{GeV}$. There are only a few correlations/negative correlations in the MP and IP at both frequencies. They occur at different time lags and are within $2.5 \sigma$ of the mean for a correlation with randomized data. This suggests that although increased pair production in the magnetosphere may contribute to GP occurrence, it is not a dominant factor. More likely possibilities for GP generation are increased coherence or changes in beaming.

Multifrequency correlations have been searched for previously. Shearer et al. (2003) found a $3 \%$ increase in the brightness of optical pulses at the time of GPs and Collins et al. (2012) found a slight correlation between GPs and enhanced optical pulses, which supports our $\sim 2 \sigma$ correlations/negative correlations in suggesting that there are small fluctuations in the magnetospheric particle density during GPs. The negative correlations, however, would suggest that increased particle density would lead to more radio emission and less $\gamma$-ray emission, which does not make sense in the context of Lyutikov's theory. Since both the positive and negative correlations are on the level of $3 \sigma$, we assume that these correlations are insignificant. Lundgren et al. (1995) found that the $\gamma$-ray flux does not vary by more than 2.5 times the average flux during a GP. Bilous et al. (2011) ruled out a strong correlation between GPs at 8.9 $\mathrm{GHz}$ and $\gamma$-ray photons above $100 \mathrm{MeV}$. They were able to put an upper limit on the $\gamma$-ray flux during IP GPs of 8-16 times the average pulsed flux, suggesting that there still might be a slight correlation between GPs and $\gamma$-ray photons. We 
could not carry out the same experiment because we did not have sufficient $\gamma$-ray photons to compare the $\gamma$-ray profile made with $\gamma$-ray photons around GPs with the $\gamma$-ray profile excluding $\gamma$-ray photons around GPs.

The recent $\gamma$-ray flare from the Crab Nebula occurred during the span of our observations, so we were able to compare the behavior of the Crab pulsar before and after the flare. We found no significant changes in pulse shape, power-law index, or $\gamma$-ray correlation, suggesting that there was no change in the properties of the pulsar during the flare, suggesting that the flare was likely caused by magnetic reconnection and not associated with the pulsar. 


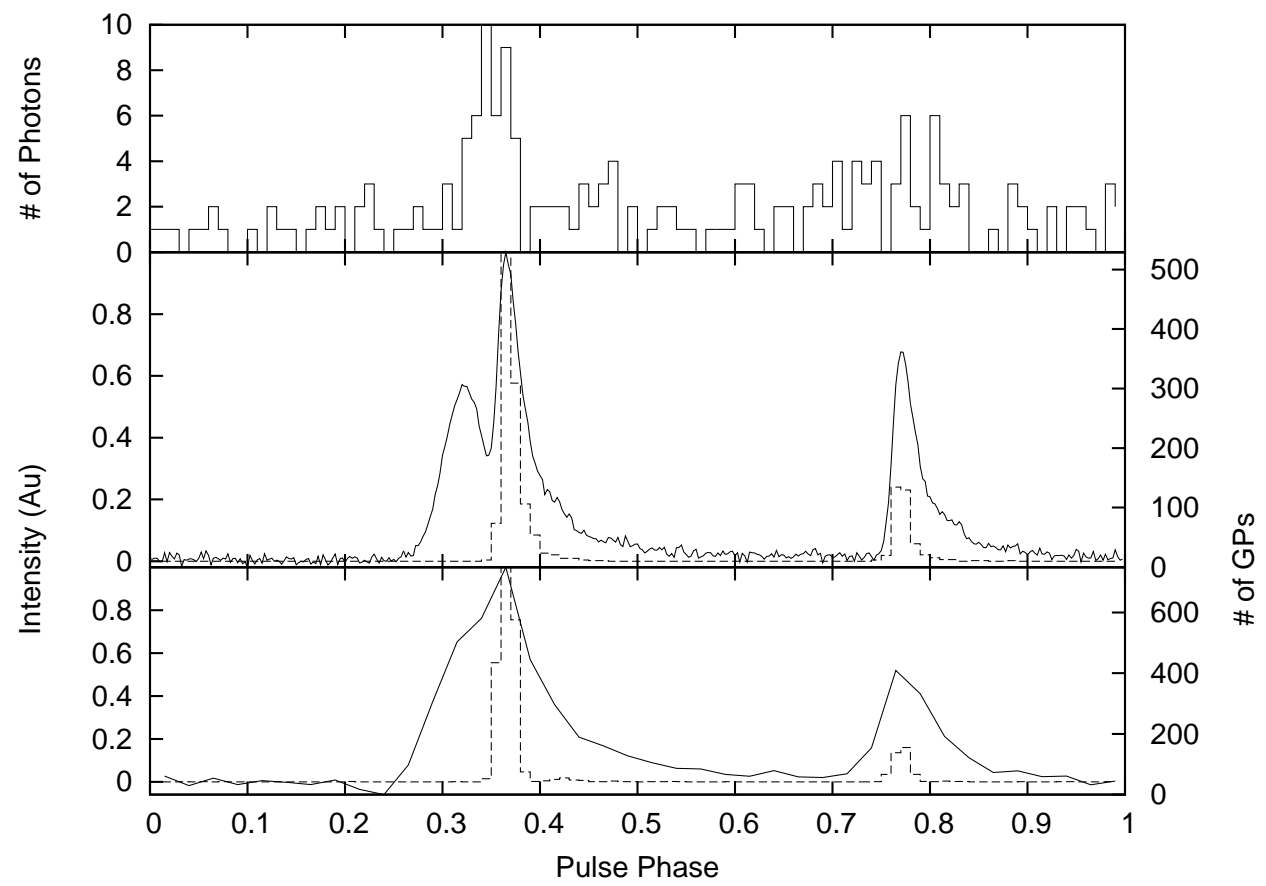

Figure 2.1: Folded pulse profiles from MJD 55406 for Fermi (top), the GBT (middle, solid line), and the GB43 (bottom, solid line), as well as histograms of the number of GPs for the GBT (middle, dashed line) and GB43 (bottom, dashed line). The Fermi profile is made using photons above $100 \mathrm{MeV}$ over a 24 hour period $(\sim 11$ hours on source time). The GB43 observations were taken at a center frequency of $330 \mathrm{MHz}$ over a $220 \mathrm{MHz}$ band for 3.7 hours and the GBT observations were taken at a center frequency of $350 \mathrm{MHz}$ over a $100 \mathrm{MHz}$ band for 10 minutes. The folded radio profiles have been normalized to have peaks of unity. 


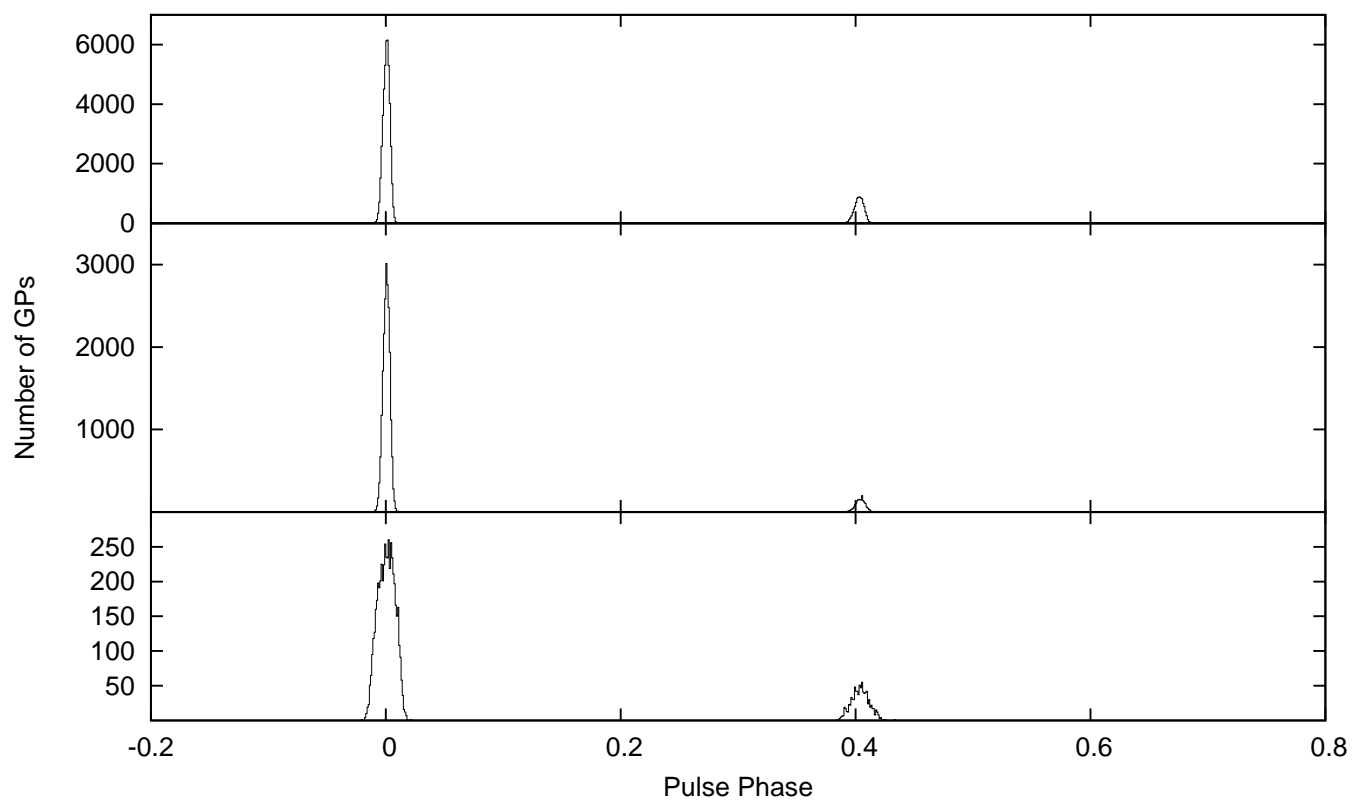

Figure 2.2: Number of GPs versus pulse phase for all GPs collected with the GB43 at $1.2 \mathrm{GHz}$ (top, post-flare; middle, pre-flare) and $330 \mathrm{MHz}$ (bottom). Most GPs at frequencies below $5 \mathrm{GHz}$ come at the phase $(\sim 0)$ of the MP, while there are still a considerable number at the IP phase $(\sim 0.4)$. No GPs are seen at other phases. The FWHMs of the $1.2 \mathrm{GHz}$ distributions (226 $\mu$ s pre-flare, $292 \mu$ s post-flare) are on the order of the FWHMs of the folded profiles at $1.2 \mathrm{GHz}$, while the FWHM of the $330 \mathrm{MHz}$ distribution $(704 \mu \mathrm{s})$ is much narrower than the folded profile and is on the order of the average GP profile at $330 \mathrm{MHz}$. Even though there were only four observing epochs after the flare, there were many more GPs recorded than pre-flare due to a large increase in receiver sensitivity. 


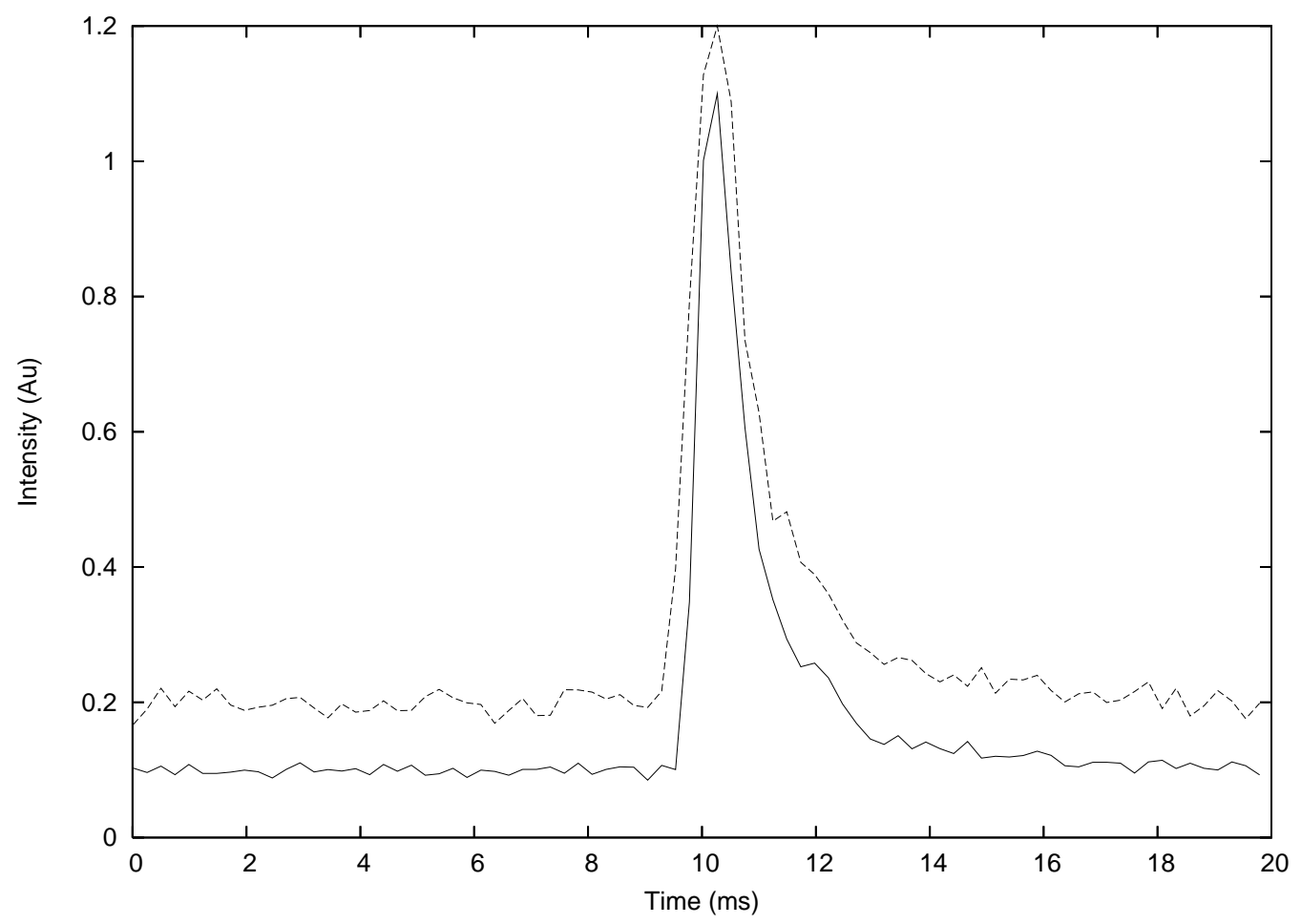

Figure 2.3: A single GP observed both with the GB43 (dashed line) at $330 \mathrm{MHz}$ and the GBT (solid line) at $350 \mathrm{MHz}$ on MJD 55406. When corrected for the dispersion delay due to different observing frequencies, the peaks of the GP from both observations are within one $\mu$ s of each other. The GB43 profile has been shifted upwards for clarity. 


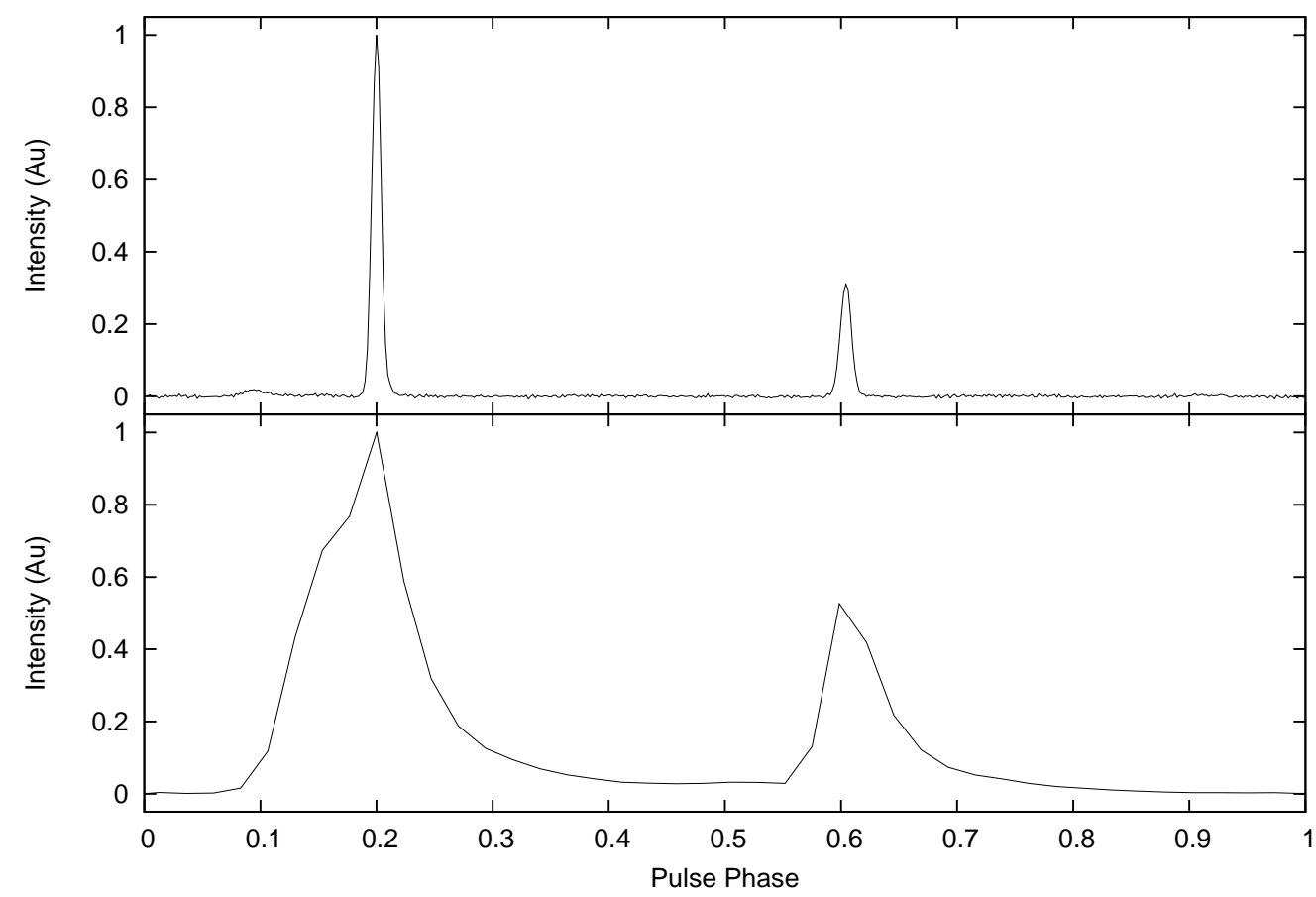

Figure 2.4: Average pulse profiles from all $1.2 \mathrm{GHz}$ (top) and $330 \mathrm{MHz}$ (bottom) observations with the GB43, listed in Table 2.1. The $1.2 \mathrm{GHz}$ profile excludes MJDs 55097, 55257, 55290, 55299, and 55346, where the folded pulse profiles were dominated by RFI. At $1.2 \mathrm{GHz}$ the MP (larger) and IP (smaller) are apparent, and a low-frequency component can be seen one-tenth of a pulse phase ahead of the MP (e.g. Moffett \& Hankins, 1996). At $330 \mathrm{MHz}$ the MP and IP are much wider due to large DM smearing, which at the top of the band $(440 \mathrm{MHz})$ is $0.3 \mathrm{~ms}$ and at the bottom $(220 \mathrm{MHz})$ is $2.4 \mathrm{~ms}$. The scattering time, estimated from Kuz'min et al. (2011), is $470 \mu \mathrm{s}$ at the top of the band and $5.8 \mathrm{~ms}$ at the bottom, and the sampling time is $819.2 \mu \mathrm{s}$. This leads to an effective resolution of $990 \mu \mathrm{s}$ at the top of the band and $6.3 \mathrm{~ms}$ at the bottom. 


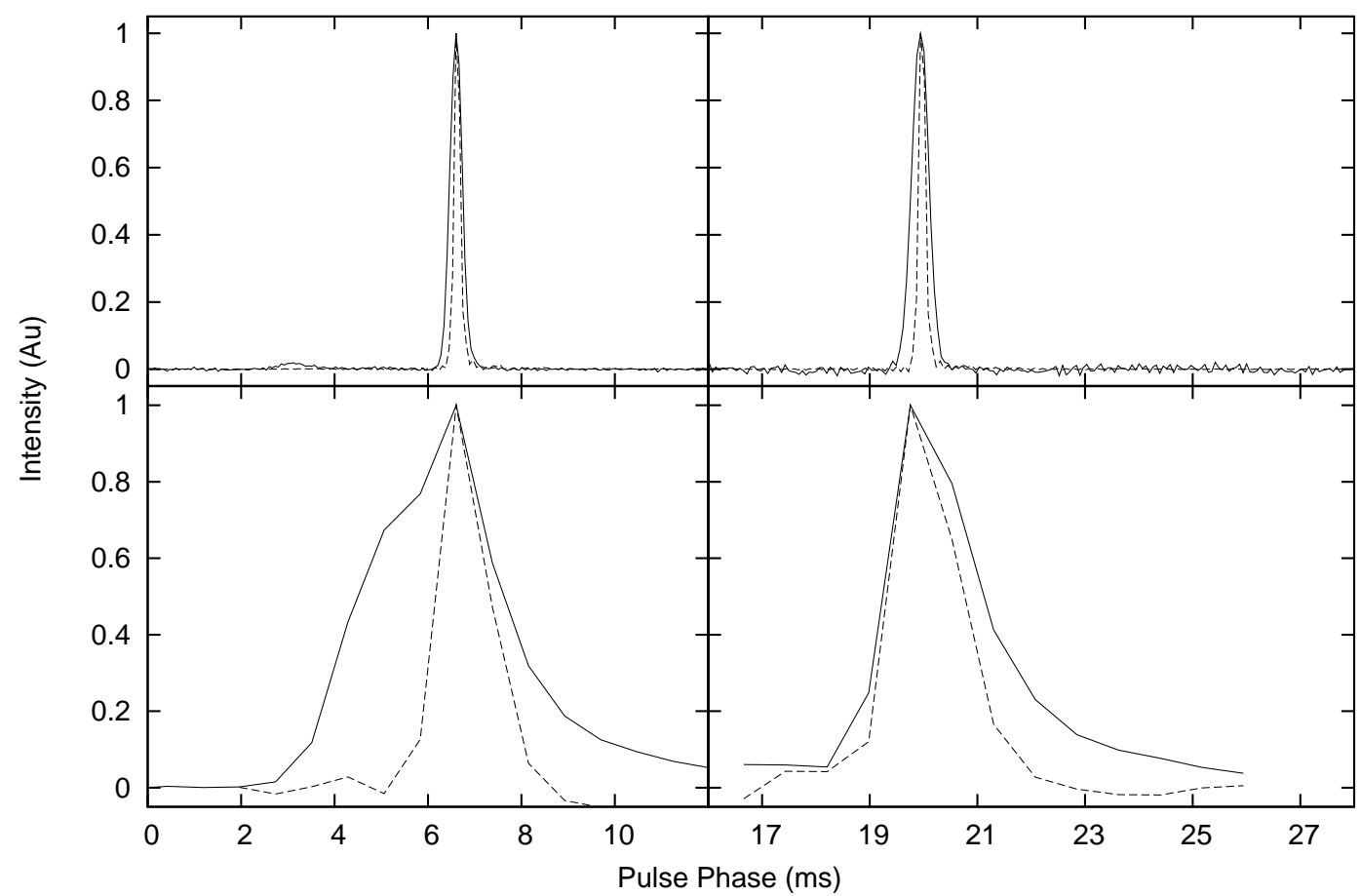

Figure 2.5: Average GP profiles for all MP (left) and IP (right) GPs (dashed lines) collected with the GB43 at $1.2 \mathrm{GHz}$ (top) and $330 \mathrm{MHz}$ (bottom). The $1.2 \mathrm{GHz}$ profiles are made by summing the 76707 individual MP and 10871 individual IP GP profiles, and the $330 \mathrm{MHz}$ profiles are made by summing the 5232 individual MP and 888 individual IP GP profiles. They are shown with the folded MP and IP profiles for all $1.2 \mathrm{GHz}$ and $330 \mathrm{MHz}$ data (solid lines). The FWHM of the $1.2 \mathrm{GHz}$ folded MP and IP are $\sim 305 \mu$ s and $\sim 360 \mu$ s, respectively, while the FWHM of the average MP and IP GPs are both $140 \mu$ s. The FWHM of the $330 \mathrm{MHz}$ folded MP and IP are $\sim 3.2 \mathrm{~ms}$ and $\sim 1.5 \mathrm{~ms}$, respectively, while the FWHM of the average MP and IP GPs are $\sim 1.1 \mathrm{~ms}$. The $330 \mathrm{MHz}$ MP is much wider than the IP due to its overlap with the MP precursor. The intensities of the profiles are arbitrary and are scaled for clarity. 


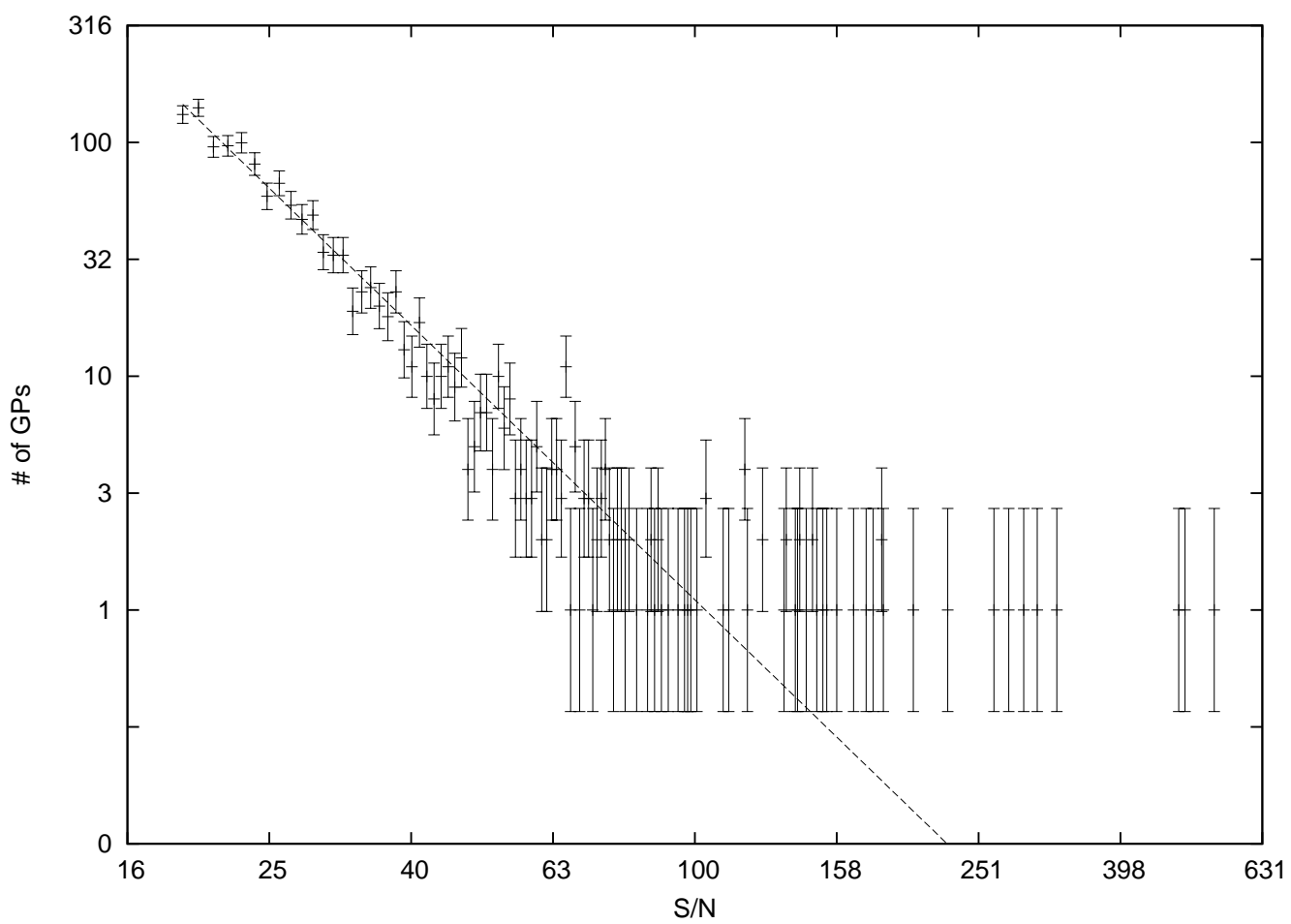

Figure 2.6: A plot of the log of the number of GPs collected with the GB43 vs the $\log$ of their $\mathrm{S} / \mathrm{N}$ for the MP at $1.2 \mathrm{GHz}$ on MJD 55099 and a best-fit power-law, which has a slope of $2.93 \pm 0.07$. The deviation from the power-law distribution at high $\mathrm{S} / \mathrm{N}$ is seen on all days, and these GPs may be examples of supergiant pulses, seen previously by Cordes et al. (2004). The average supergiant pulse is about 15 times stronger than the average GP. 


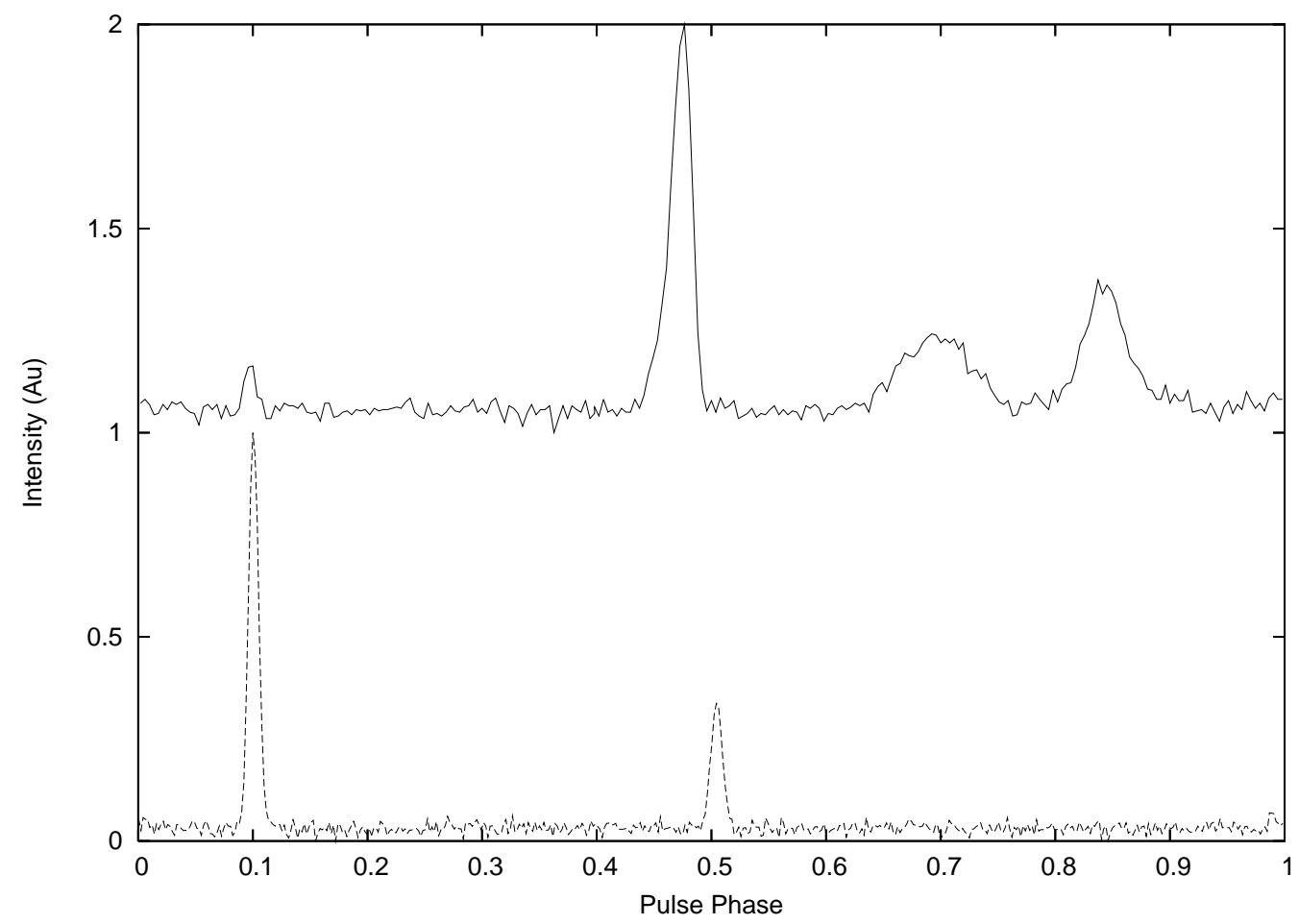

Figure 2.7: Average profiles from the GBT at $8.9 \mathrm{GHz}$ (solid line, from Bilous et al. (2011)) and the GB43 at $1.2 \mathrm{GHz}$ (dashed line). The weakening of the MP at high frequencies can be seen, as well as the strengthening and shift of the IP. 


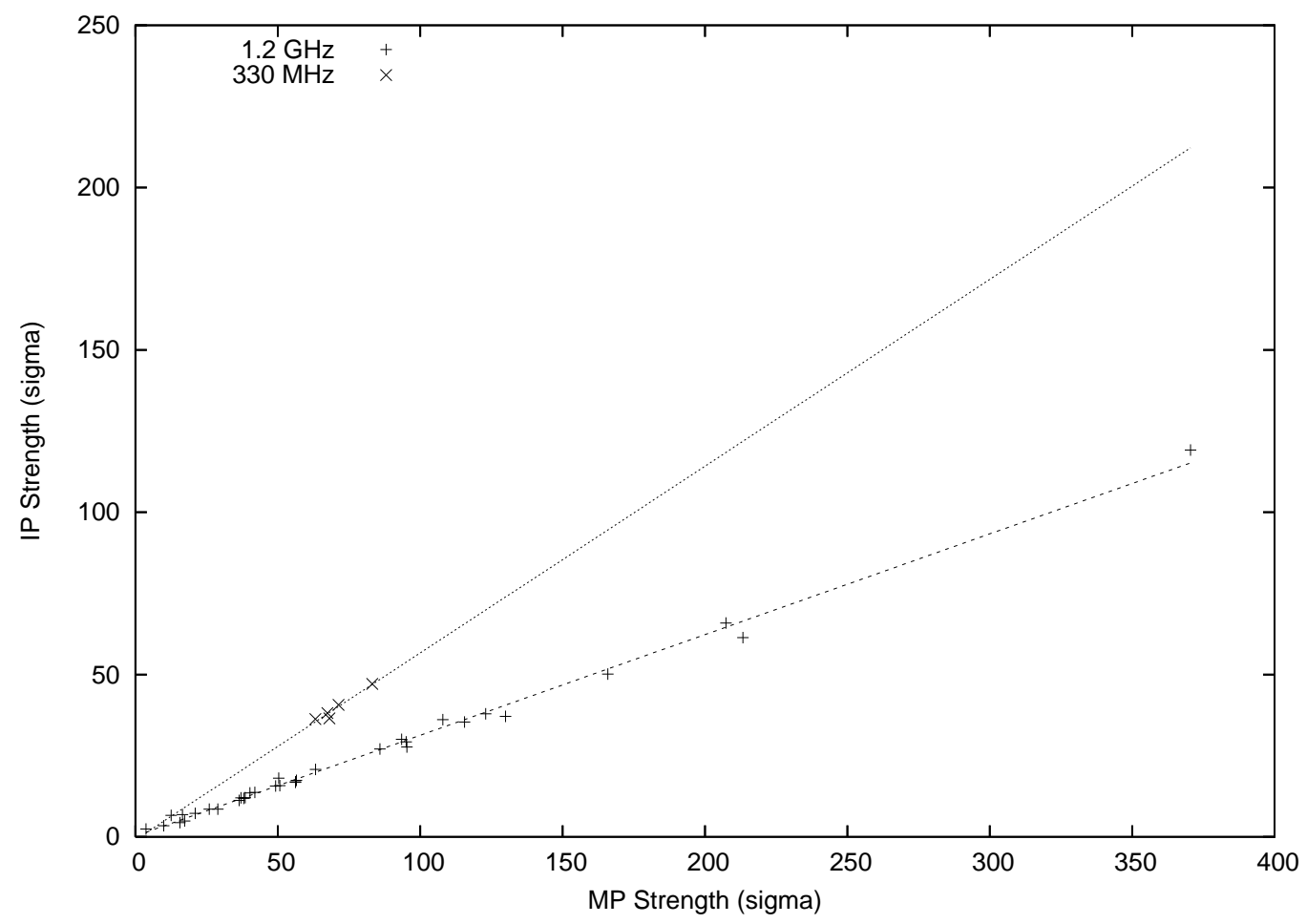

Figure 2.8: Comparison of the strength of the MP of the folded profile to that of the IP for each observation. There is an obvious linear trend in both the $330 \mathrm{MHz}$ and $1.2 \mathrm{GHz}$ data, with slopes of $0.58 \pm 0.08$ and $0.310 \pm 0.004$, respectively. This supports the theory that the IP may be a reflection of the MP off of the magnetosphere. 


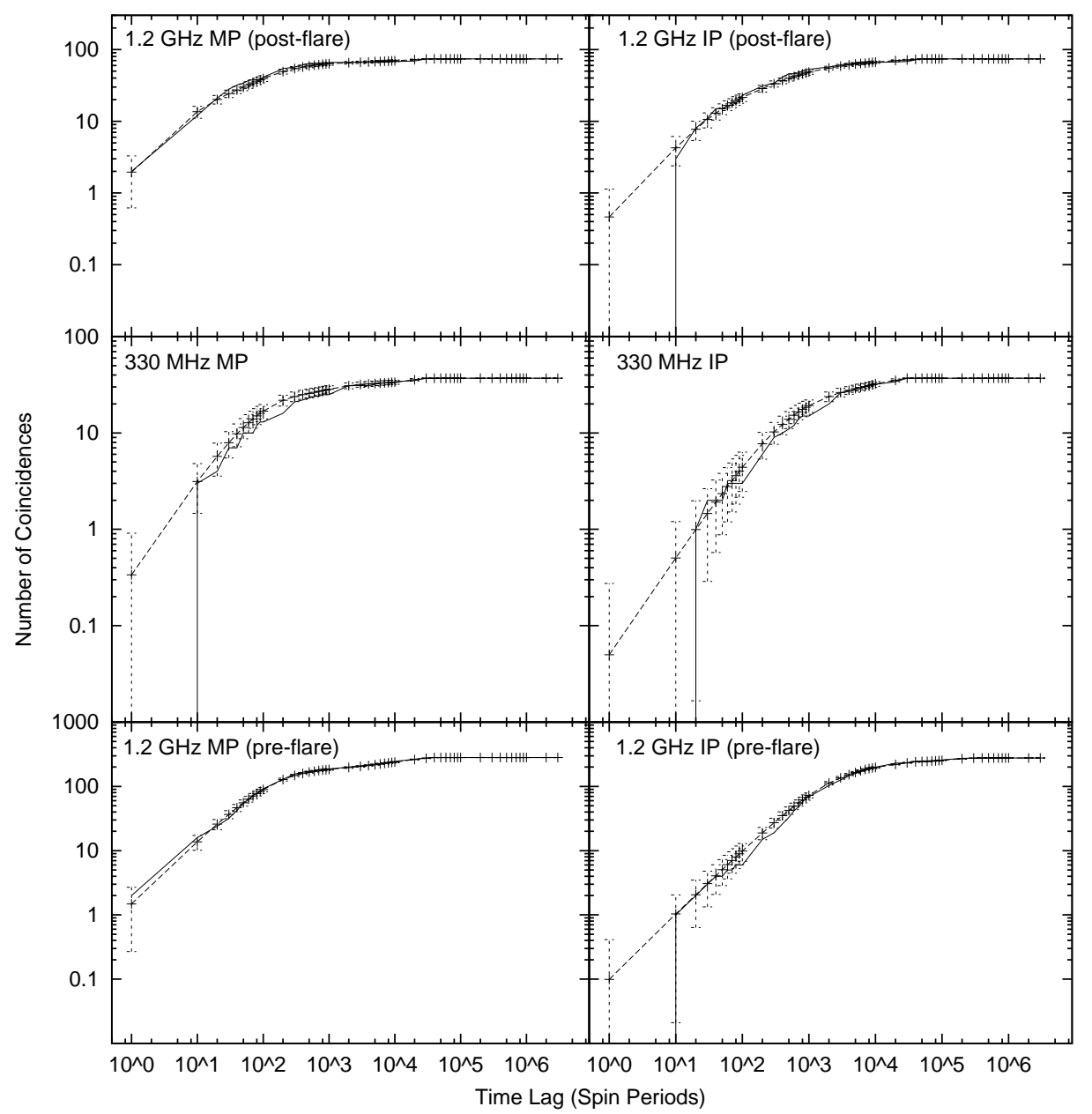

Figure 2.9: Number of coincidences between Fermi photons and GB43 GP TOAs (solid lines), as well as the number of coincidences between randomized Fermi photons and GP TOAs (dotted line) with $1 \sigma$ error bars. The bottom row is the correlation with the $1.2 \mathrm{GHz}$ pre-flare data, the middle row is the $330 \mathrm{MHz}$ data, and the top row is the $1.2 \mathrm{GHz}$ post-flare data. For each row the MP is the left panel and the IP is the right panel. 


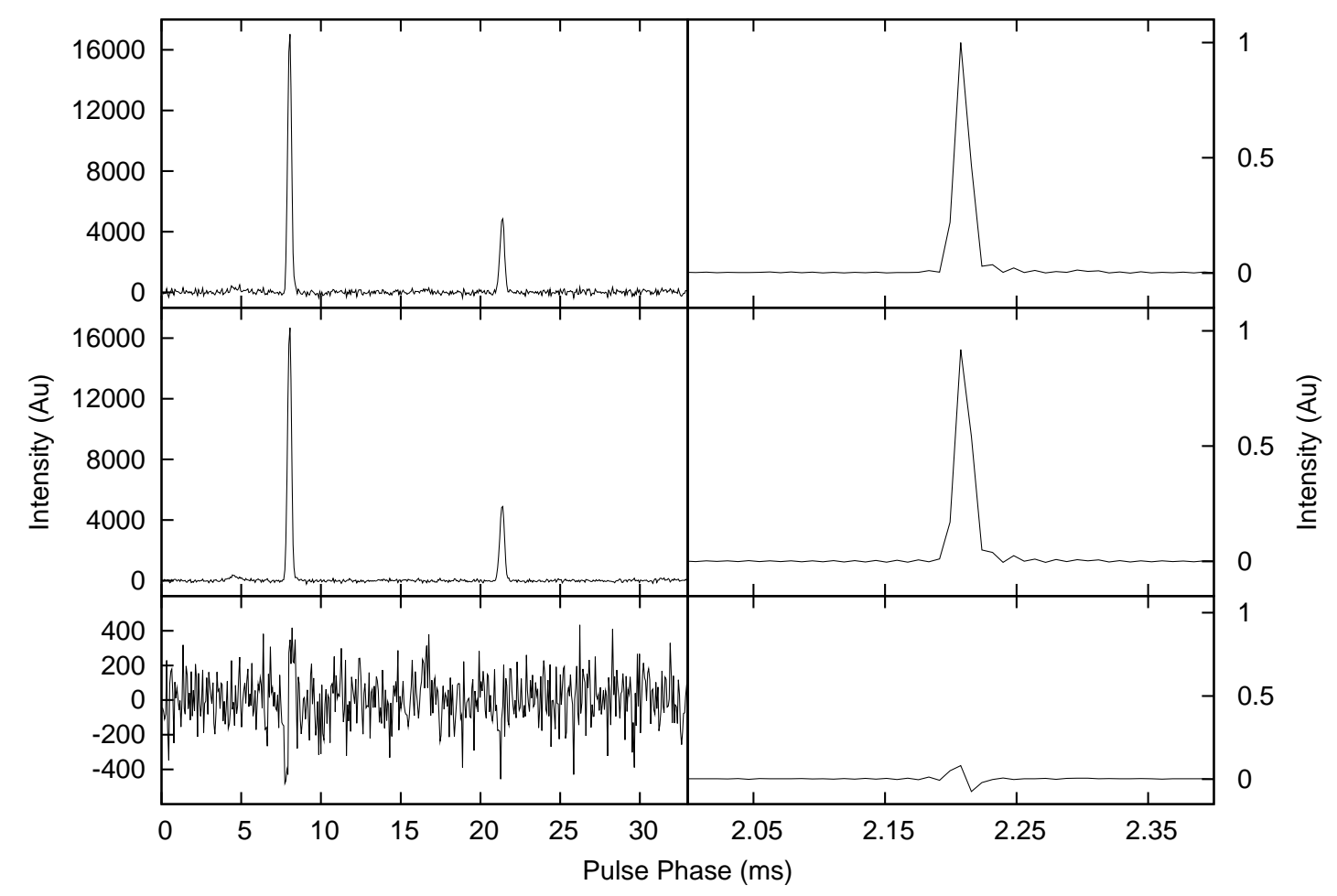

Figure 2.10: A $\gamma$-ray flare occurred from MJDs 55457-55461, so we checked for changes in the average pulse shape and GP shape. Left: The folded pulse profiles from 1.2 GHz GB43 observations on MJD 55352 (top) and MJD 55532 (middle), and the difference between them (bottom). The profiles are scaled so that the area under the main pulse for both profiles is the same. As can be seen from the bottom plot, the difference is on the order of the RMS noise, so there is no statistically significant change in pulse shape. Right: The average GP profiles from 1.2 GHz GB43 observations on MJD 55352 (top) and MJD 55532 (middle), and the difference between them (bottom). This difference is not significant as there is a larger variation in average GP shape between pre-flare days. The profiles are also scaled so that the area under the main pulse for both profiles is the same. 
Table 2.1. Observational parameters including the MJD the observation was taken, the center frequency used, the length of the observation, the sampling time, the DM used for dedispersion, and the number of GPs found with $S / N \geq 10$. The starred MJDs are days in which simultaneous observations with the GBT occurred.

\begin{tabular}{|c|c|c|c|c|c|c|}
\hline MJD & $\begin{array}{c}\text { Frequency } \\
(\mathrm{MHz})\end{array}$ & $\begin{array}{l}\text { Observation } \\
\text { Length (min) }\end{array}$ & $\begin{array}{l}\text { Sampling } \\
\text { Time }(\mu \mathrm{s})\end{array}$ & 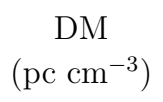 & $\begin{array}{c}\text { Number } \\
\text { of MP GPs }\end{array}$ & $\begin{array}{l}\text { Number } \\
\text { of IP GPs }\end{array}$ \\
\hline 55079 & 1200 & 5 & 204.8 & 56.8005 & 33 & 1 \\
\hline 55079 & 1200 & 108 & 204.8 & 56.8005 & 341 & 27 \\
\hline$* 55083$ & 1200 & 4 & 204.8 & 56.8005 & 19 & 1 \\
\hline$* 55083$ & 1200 & 120 & 204.8 & 56.8005 & 582 & 42 \\
\hline 55085 & 1200 & 120 & 204.8 & 56.8005 & 73 & 0 \\
\hline$* 55086$ & 1200 & 156 & 204.8 & 56.8005 & 1677 & 96 \\
\hline$* 55090$ & 1200 & 150 & 204.8 & 56.8005 & 551 & 31 \\
\hline$* 55093$ & 1200 & 168 & 204.8 & 56.8005 & 650 & 42 \\
\hline$* 55095$ & 1200 & 108 & 51.2 & 56.8005 & 669 & 47 \\
\hline *55096 & 1200 & 150 & 204.8 & 56.8005 & 264 & 21 \\
\hline$* 55097$ & 1200 & 240 & 204.8 & 56.8005 & 519 & 45 \\
\hline$* 55099$ & 1200 & 210 & 204.8 & 56.8005 & 1435 & 76 \\
\hline *55102 & 1200 & 150 & 204.8 & 56.8005 & 1228 & 76 \\
\hline 55118 & 1200 & 6 & 204.8 & 56.8109 & 2 & 0 \\
\hline 55118 & 1200 & 6 & 51.2 & 56.8109 & 16 & 0 \\
\hline 55139 & 1200 & 240 & 51.2 & 56.8229 & 3757 & 308 \\
\hline 55142 & 1200 & 120 & 51.2 & 56.8229 & 244 & 22 \\
\hline 55172 & 1200 & 22 & 51.2 & 56.8279 & 25 & 0 \\
\hline 55178 & 1200 & 282 & 51.2 & 56.8279 & 1808 & 110 \\
\hline 55180 & 1200 & 114 & 51.2 & 56.8279 & 478 & 27 \\
\hline 55240 & 1200 & 7 & 245.76 & 56.8053 & 32 & 2 \\
\hline 55240 & 1200 & 180 & 122.88 & 56.8053 & 1575 & 101 \\
\hline 55256 & 1200 & 15 & 61.44 & 56.8622 & 148 & 8 \\
\hline 55257 & 1200 & 198 & 245.76 & 56.8622 & 1098 & 76 \\
\hline 55261 & 1200 & 492 & 245.76 & 56.8622 & 41 & 9 \\
\hline 55264 & 1200 & 15 & 61.44 & 56.8622 & 106 & 5 \\
\hline 55269 & 1200 & 360 & 245.76 & 56.8622 & 42 & 2 \\
\hline 55270 & 1200 & 30 & 245.76 & 56.8622 & 291 & 17 \\
\hline 55284 & 1200 & 180 & 245.76 & 56.8622 & 1094 & 63 \\
\hline 55290 & 1200 & 108 & 245.76 & 56.8228 & 169 & 10 \\
\hline 55299 & 1200 & 10 & 245.76 & 56.8228 & 4 & 0 \\
\hline 55304 & 1200 & 60 & 245.76 & 56.8228 & 73 & 5 \\
\hline 55346 & 1200 & 60 & 245.76 & 56.8022 & 672 & 40 \\
\hline 55347 & 1200 & 120 & 245.76 & 56.8022 & 1170 & 76 \\
\hline 55347 & 1200 & 168 & 61.44 & 56.8022 & 2701 & 243 \\
\hline 55352 & 1200 & 240 & 61.44 & 56.8022 & 2182 & 146 \\
\hline
\end{tabular}


Table 2.1-Continued

\begin{tabular}{ccccccc}
\hline \hline MJD & $\begin{array}{c}\text { Frequency } \\
(\mathrm{MHz})\end{array}$ & $\begin{array}{c}\text { Observation } \\
\text { Length }(\mathrm{min})\end{array}$ & $\begin{array}{c}\text { Sampling } \\
\text { Time }(\mu \mathrm{s})\end{array}$ & $\begin{array}{c}\mathrm{DM} \\
\left(\mathrm{pc} \mathrm{cm}^{-3}\right)\end{array}$ & $\begin{array}{c}\text { Number } \\
\text { of MP GPs }\end{array}$ & $\begin{array}{c}\text { Number } \\
\text { of IP GPs }\end{array}$ \\
\hline 55403 & 330 & 37 & 223.42 & 56.7988 & 271 & 31 \\
55405 & 330 & 60 & 819.2 & 56.7988 & 1201 & 250 \\
55406 & 330 & 222 & 819.2 & 56.7988 & 1775 & 273 \\
55411 & 330 & 60 & 819.2 & 56.7962 & 1332 & 220 \\
55412 & 330 & 30 & 819.2 & 56.7962 & 653 & 114 \\
55516 & 1200 & 550 & 245.76 & 56.8065 & 4870 & 326 \\
55532 & 1200 & 315 & 61.44 & 56.7964 & 11671 & 937 \\
55539 & 1200 & 10 & 61.44 & 56.7964 & 786 & 146 \\
55541 & 1200 & 335 & 61.44 & 56.7964 & 33611 & 7687 \\
\hline
\end{tabular}


Table 2.2: Sampling times, DM smearing times, scattering times, and effective resolution at the top and bottom of the band for the GB43 $330 \mathrm{MHz}$ and $1.2 \mathrm{GHz}$ observations, as well as for the GBT $350 \mathrm{MHz}$ observation. The DM smearing times were calculated using the delay between two consecutive channels caused by dispersion, and the scattering times were estimated from Kuz'min et al. (2011). The sampling time listed for the $1.2 \mathrm{GHz}$ GB43 observation was the most common sampling time from Table 2.1.

\begin{tabular}{cccccccc}
\hline \hline $\begin{array}{c}\text { Frequency } \\
(\mathrm{MHz})\end{array}$ & $\begin{array}{c}\text { Sampling Time } \\
\text { (us) }\end{array}$ & \multicolumn{2}{c}{$\begin{array}{c}\text { DM Smearing Time } \\
\text { Top }\end{array}$} & Bottom & \multicolumn{2}{c}{$\begin{array}{c}\text { Scattering Time } \\
\text { Top }\end{array}$} & \multicolumn{2}{c}{ Bottom } & Top & Bottom \\
\hline $330(\mathrm{~GB} 43)$ & 819.2 & $300 \mu \mathrm{s}$ & $2.4 \mathrm{~ms}$ & $470 \mu \mathrm{s}$ & $5.8 \mathrm{~ms}$ & $990 \mu \mathrm{s}$ & $6.3 \mathrm{~ms}$ \\
$350(\mathrm{GBT})$ & 81.92 & $361 \mu \mathrm{s}$ & $855 \mu \mathrm{s}$ & $670 \mu \mathrm{s}$ & $1.9 \mathrm{~ms}$ & $765 \mu \mathrm{s}$ & $2.0 \mathrm{~ms}$ \\
$1200(\mathrm{~GB} 43)$ & 204.8 & $23 \mu \mathrm{s}$ & $180 \mu \mathrm{s}$ & $4 \mu \mathrm{s}$ & $54 \mu \mathrm{s}$ & $206 \mu \mathrm{s}$ & $278 \mu \mathrm{s}$ \\
\hline
\end{tabular}


Table 2.3. Differential power-law measurements for the MP and IP for each observing epoch. Some MJDs have two entries listed because there were two separate observations on those days. An X means that there were not enough pulses recorded for the MP or IP on that day to fit a power-law to the amplitude distribution.

\begin{tabular}{|c|c|c|}
\hline MJD & $\begin{array}{c}\text { Differential Power- } \\
\text { law Index (MP) }\end{array}$ & $\begin{array}{c}\text { Differential Power- } \\
\text { law Index (IP) }\end{array}$ \\
\hline 55079 & $\mathrm{X}$ & $\mathrm{X}$ \\
\hline 55079 & $2.1 \pm 0.3$ & $\mathrm{X}$ \\
\hline 55083 & $\mathrm{X}$ & $\mathrm{X}$ \\
\hline 55083 & $3.1 \pm 0.2$ & $\mathrm{X}$ \\
\hline 55085 & $\mathrm{X}$ & $\mathrm{X}$ \\
\hline 55086 & $2.70 \pm 0.06$ & $\mathrm{X}$ \\
\hline 55090 & $2.8 \pm 0.1$ & $\mathrm{X}$ \\
\hline 55093 & $2.6 \pm 0.2$ & $\mathrm{X}$ \\
\hline 55095 & $2.9 \pm 0.2$ & $\mathrm{X}$ \\
\hline 55096 & $\mathrm{X}$ & $\mathrm{X}$ \\
\hline 55097 & $\mathrm{X}$ & $\mathrm{X}$ \\
\hline 55099 & $2.93 \pm 0.07$ & $\mathrm{X}$ \\
\hline 55102 & $2.92 \pm 0.08$ & $\mathrm{X}$ \\
\hline 55118 & $\mathrm{X}$ & $\mathrm{X}$ \\
\hline 55118 & $\mathrm{X}$ & $\mathrm{X}$ \\
\hline 55139 & $2.61 \pm 0.08$ & $\mathrm{X}$ \\
\hline 55142 & $\mathrm{X}$ & $\mathrm{X}$ \\
\hline 55172 & $\mathrm{X}$ & $\mathrm{X}$ \\
\hline 55178 & $2.81 \pm 0.08$ & $\mathrm{X}$ \\
\hline 55180 & $\mathrm{X}$ & $\mathrm{X}$ \\
\hline 55240 & $\mathrm{X}$ & $\mathrm{X}$ \\
\hline 55240 & $2.1 \pm 0.1$ & $\mathrm{X}$ \\
\hline 55256 & $\mathrm{X}$ & $\mathrm{X}$ \\
\hline 55257 & $2.1 \pm 0.1$ & $\mathrm{X}$ \\
\hline 55261 & $\mathrm{X}$ & $\mathrm{X}$ \\
\hline 55264 & $\mathrm{X}$ & $\mathrm{X}$ \\
\hline 55269 & $\mathrm{X}$ & $\mathrm{X}$ \\
\hline 55270 & $\mathrm{X}$ & $\mathrm{X}$ \\
\hline 55284 & $2.1 \pm 0.1$ & $\mathrm{X}$ \\
\hline 55290 & $\mathrm{X}$ & $\mathrm{X}$ \\
\hline 55299 & $\mathrm{X}$ & $\mathrm{X}$ \\
\hline 55304 & $\mathrm{X}$ & $\mathrm{X}$ \\
\hline 55346 & $2.4 \pm 0.2$ & $\mathrm{X}$ \\
\hline 55347 & $2.8 \pm 0.1$ & $\mathrm{X}$ \\
\hline 55347 & $2.40 \pm 0.07$ & $\mathrm{X}$ \\
\hline 55352 & $2.21 \pm 0.07$ & $\mathrm{X}$ \\
\hline
\end{tabular}


Table 2.3-Continued

\begin{tabular}{ccc}
\hline \hline MJD & $\begin{array}{c}\text { Differential Power- } \\
\text { law Index (MP) }\end{array}$ & $\begin{array}{c}\text { Differential Power- } \\
\text { law Index (IP) }\end{array}$ \\
\hline 55403 & $2.5 \pm 0.2$ & $\mathrm{X}$ \\
55405 & $2.95 \pm 0.09$ & $2.4 \pm 0.2$ \\
55406 & $2.94 \pm 0.05$ & $3.1 \pm 0.2$ \\
55411 & $2.8 \pm 0.1$ & $2.9 \pm 0.2$ \\
55412 & $2.7 \pm 0.2$ & $\mathrm{X}$ \\
55516 & $2.93 \pm 0.05$ & $2.4 \pm 0.4$ \\
55532 & $2.56 \pm 0.05$ & $2.7 \pm 0.2$ \\
55541 & $2.8 \pm 0.1$ & $2.81 \pm 0.03$ \\
\hline
\end{tabular}


Table 2.4: Comparison of differential power-law indices for the MP and IP at both 1.2 $\mathrm{GHz}$ and $330 \mathrm{MHz}$ between this work and previously published values.

\begin{tabular}{|c|c|c|c|}
\hline $\begin{array}{l}\text { Frequency } \\
(\mathrm{MHz})\end{array}$ & $\begin{array}{l}\text { Differential Power- } \\
\text { law Index (MP) }\end{array}$ & $\begin{array}{l}\text { Differential Power- } \\
\text { law Index (IP) }\end{array}$ & Reference \\
\hline 112 & $3.3^{\mathrm{a}}$ & - & Smirnova \& Logvinenko (2009) \\
\hline 146 & 3.5 & 3.8 & Argyle \& Gower (1972) \\
\hline 200 & $2.7^{\mathrm{a}}$ & - & Bhat et al. (2007) \\
\hline 330 & $2.5-3.0$ & $2.4-3.1$ & This work \\
\hline 430 & 2.3 & $-{ }^{b}$ & Cordes et al. (2004) \\
\hline 600 & 3.2 & 3.0 & Popov et al. (2009) \\
\hline 812 & $3.3^{\mathrm{a}}$ & - & Lundgren et al. (1995) \\
\hline 1200 & $2.7-4.2$ & 2.6 & Popov \& Stappers (2007) \\
\hline 1200 & $2.1-3.1$ & $2.4-2.8$ & This work \\
\hline 1300 & $2.3^{\mathrm{a}}$ & - & Bhat et al. (2008) \\
\hline 1400 & 2.8 & 3.1 & Karuppusamy et al. (2010) \\
\hline 2100 & $3.0^{\mathrm{a}}$ & - & Zhuravlev et al. (2011) \\
\hline 4850 & $2.8^{\mathrm{a}}$ & - & Popov et al. (2008) \\
\hline
\end{tabular}

${ }^{\text {a }} \mathrm{MP}$ and IP GPs were combined in these analyses

b No measurement was taken for the IP

Table 2.5: Differential power-law indices for different source classes for comparison with GP power-laws.

\begin{tabular}{ccc}
\hline \hline $\begin{array}{c}\text { Source } \\
\text { Class }\end{array}$ & $\begin{array}{c}\text { Power-law } \\
\text { Index }\end{array}$ & Reference \\
\hline Normal Pulsars & 3.85 & Kramer et al. (2002) \\
Magnetars & $2.1-7.7$ & Serylak et al. (2009) \\
RRATs & 3.0 & Miller et al. (2011) \\
\hline
\end{tabular}


Table 2.6: Maximum correlation/negative correlation for each frequency at each energy cut, and the time lags at which they occur. Note that the maximum correlation for the $330 \mathrm{MHz}$ IP with the $500 \mathrm{MeV}$ energy cut is negative.

\begin{tabular}{|c|c|c|c|c|c|c|}
\hline $\begin{array}{l}\text { Energy } \\
\text { Cut }\end{array}$ & $\begin{array}{l}\text { Frequency } \\
\quad(\mathrm{MHz})\end{array}$ & $\mathrm{MP} / \mathrm{IP}$ & $\begin{array}{c}\text { Max } \\
\text { Correlation }(\sigma)\end{array}$ & $\begin{array}{c}\text { Time Lag } \\
\text { (Spin Periods) }\end{array}$ & $\begin{array}{c}\text { Max Anti- } \\
\text { correlation }(\sigma)\end{array}$ & $\begin{array}{c}\text { Time Lag } \\
\text { (Spin Periods) }\end{array}$ \\
\hline \multirow{6}{*}{$100 \mathrm{MeV}$} & \multirow{2}{*}{ Pre-flare 1200} & $\mathrm{MP}$ & +1.4 & 30000 & -0.9 & 30 \\
\hline & & IP & +2.1 & 200000 & -1.7 & 300 \\
\hline & \multirow{2}{*}{330} & MP & +0.2 & 10000 & -2.1 & 200 \\
\hline & & IP & +0.5 & 30 & -1.5 & 1000 \\
\hline & \multirow{2}{*}{ Post-flare 1200} & MP & +1.5 & 40 & -2.4 & 20000 \\
\hline & & $\mathrm{IP}$ & +1.9 & 500 & -2.3 & 30000 \\
\hline \multirow{6}{*}{$500 \mathrm{MeV}$} & \multirow{2}{*}{ Pre-flare 1200} & $\mathrm{MP}$ & +2.3 & 1 & -0.4 & 8000 \\
\hline & & IP & +1.5 & 20000 & -1.5 & 700 \\
\hline & \multirow{2}{*}{330} & MP & +0.1 & 6000 & -1.7 & 200 \\
\hline & & IP & -0.1 & 300 & -1.7 & 2000 \\
\hline & \multirow{2}{*}{ Post-flare 1200} & MP & +0.8 & 200 & -3.5 & 20000 \\
\hline & & IP & +1.0 & 200 & -2.7 & 30000 \\
\hline \multirow{6}{*}{$1 \mathrm{GeV}$} & \multirow{2}{*}{ Pre-flare 1200} & $\mathrm{MP}$ & +3.2 & 1 & -0.1 & 60 \\
\hline & & IP & +2.0 & 5000 & -1.2 & 200 \\
\hline & \multirow{2}{*}{330} & MP & +0.8 & 6000 & -0.9 & 10 \\
\hline & & IP & +0.9 & 6000 & -1.1 & 100 \\
\hline & \multirow{2}{*}{ Post-flare 1200} & MP & +1.9 & 60 & -0.4 & 1 \\
\hline & & IP & +2.1 & 3000 & -0.7 & 10 \\
\hline
\end{tabular}




\section{Chapter 3}

\section{Discovery of Six New Pulsars in Archival Data}

This chapter was originally published as a refereed paper in the Astrophysical Journal by M. B. Mickaliger et al. in November 2012. PSR J1753-2822 has been added, and minor word changes from the original have been made where appropriate.

\subsection{Introduction}

While targeted searches have been useful in finding unique pulsars, most pulsars known today have been found in large-scale, blind pulsar surveys. One such survey, the Parkes Multibeam Pulsar Survey (PMPS; Manchester et al., 2001), surveyed a strip along the Galactic plane from $l=260^{\circ}$ to $l=50^{\circ}$ and with $|b|<5^{\circ}$ using the 13-beam receiver (Staveley-Smith et al., 1996) on the Parkes 64-m telescope. Initial processing of the data resulted in the discovery of 742 pulsars (Manchester et al., 2001; Morris et al., 2002; Kramer et al., 2003; Hobbs et al., 2004; Faulkner et al., 2004; Lorimer et al., 2006). Another 75 pulsars and 30 RRATs (rotating radio transients) were found in further reprocessings (Eatough et al., 2009, 2010, 2011; Keith et al., 2009; McLaughlin et al., 2006; Keane et al., 2010, 2011; Knispel et al., 2013). The additional pulsars were found due to the implementation of new techniques for removing terrestrial interference and sorting pulsar candidates.

In this Chapter, we present the discovery of a further six pulsars in the PMPS 
data. The motivation for our re-analysis of the PMPS data was a single-pulse study, which is presented in Chapter 4. Single-pulse studies involve the search for and characterization of transient, non-periodic bursts. We performed periodicity searches as well as single-pulse searches since the additional processing time was negligible. In Section 3.2, we describe the data reduction and analysis. Section 3.3 details the six pulsars that we discovered, and conclusions are given in Section 3.4.

\subsection{Data Reduction}

The PMPS data were searched for periodic signals using freely-available analysis software ${ }^{1}$. First, the frequency channels in the data were shifted to correct for the dispersion due to free electrons in the interstellar medium. This dedispersion is done at many dispersion measures (DM, which is the integrated column density of electrons along the line of sight) and results in a time series for each DM. The total number of DMs searched was 203 and was optimally chosen by the dedisperse_all ${ }^{2}$ program, which we used for dedispersion due to its speed and efficiency. The time series were processed by seek, a program that searches for periodic signals from an object. We searched for both periodic signals and single pulses out to a DM of $5000 \mathrm{pc} \mathrm{cm}^{-3}$. This upper limit was chosen in order to be sensitive to highlydispersed bursts. Results from the single-pulse search are presented in Chapter 4. The periodicity-search analysis method implemented in seek is the standard Fourier-based approach (see Chapter 1) where the amplitude spectrum is subject

\footnotetext{
${ }^{1}$ http://sigproc.sourceforge.net

${ }^{2}$ http://www.github.com/swinlegion
} 
to multiple harmonic folds, summing $2,4,8$, and 16 harmonics. This process increases sensitivity to narrow pulses in a close-to-optimal fashion (Ransom et al., 2002). All candidate signals, with spectral S/Ns greater than six, are sought and saved during this process. After all DM trials have been searched, statistically significant candidates with $\mathrm{S} / \mathrm{N}$ greater than nine are subject to further analysis. For these candidates, the raw multi-channel data are then folded at the period of each candidate using prepfold, part of the PRESTO package ${ }^{3}$. The prepfold program carries out a search to optimize the period from seek and produces a set of diagnostic plots for each candidate. See Chapter 1 for an example and description of a diagnostic plot.

No acceleration searches were carried out in this reduction. To reduce the number of plots that needed to be inspected by eye, we selected candidates with periods under $50 \mathrm{~ms}$, DMs greater than $10 \mathrm{pc} \mathrm{cm}^{-3}$, and spectral S/Ns greater than nine for viewing, resulting in $\sim 270000$ candidates, six of which have already been confirmed as pulsars. Since this processing was never intended to be rigorous, we assumed that most pulsars with periods greater than $50 \mathrm{~ms}$ had been discovered, and that most candidates with a DM that peaked under $10 \mathrm{pc} \mathrm{cm}^{-3}$ were interference.

\subsection{Newly Discovered Pulsars}

From our inspection of the prepfold plots, we identified six very promising pulsar candidates and have subsequently been able to confirm these as new pulsars and perform follow-up observations as described below.

\footnotetext{
${ }^{3}$ http://www.cv.nrao.edu/ $\sim_{\text {sransom/presto }}$
} 


\subsubsection{PSR J0922-52}

PSR J0922-52 has a period of $9.68 \mathrm{~ms}$ and a DM of $122.4 \mathrm{pc} \mathrm{cm}^{-3}$. The spectral S/N and $\chi^{2}$ of the profile from the discovery observation, reported by seek and prepfold, are 9.1 and 2.7, respectively. The inferred distance from the NE2001 model (Cordes \& Lazio, 2002) is $0.8 \mathrm{kpc}$. It was confirmed on MJD 56102 with a 35 minute observation using the Parkes telescope at $1400 \mathrm{MHz}$. The full width at half maximum (FWHM) of the profile from the confirmation observation (Figure $3.1)$ is $790 \mu \mathrm{s}$. Since the position is not well constrained by the observations we have been able to carry out, we can only estimate, using the radiometer equation (Lorimer \& Kramer, 2004), a lower limit on the mean flux at $1400 \mathrm{MHz}$, which is 0.16 mJy. Further observations are needed in order to time this pulsar and determine its physical parameters.

\subsubsection{PSR J1147-66}

PSR J1147-66 has a period of $3.72 \mathrm{~ms}$ and a DM of $133.8 \mathrm{pc} \mathrm{cm}^{-3}$. The spectral $\mathrm{S} / \mathrm{N}$ and $\chi^{2}$ of the profile from the discovery observation, reported by seek and prepfold, are 10.9 and 6.0, respectively. The inferred distance from the NE2001 model is $2.7 \mathrm{kpc}$. It was confirmed on MJD 56158 with a 20 minute observation using the Parkes telescope at $1400 \mathrm{MHz}$. The FWHM of the profile from the confirmation observation (Figure 3.1) is $795 \mu \mathrm{s}$. The estimate of the lower limit on the mean flux at $1400 \mathrm{MHz}$ is $0.80 \mathrm{mJy}$. As with PSR J0922-52, further observations are needed for timing and determining its physical characteristics. 


\subsubsection{PSR J1227-6208}

PSR J1227-6208 has a period of $34.53 \mathrm{~ms}$ and a DM of $362.6 \mathrm{pc} \mathrm{cm}^{-3}$. The spectral S/N and $\chi^{2}$ of the profile from the discovery observation, reported by seek and prepfold, are 12.6 and 4.6, respectively. The inferred distance from the NE2001 model is $8.3 \mathrm{kpc}$. It was confirmed on MJD 55857 with a 15 minute observation using the Parkes telescope at $1400 \mathrm{MHz}$, and was independently detected by the High Time Resolution Universe (HTRU) pulsar survey (Keith et al., 2010), as well as the ongoing processing by Einstein@Home (Knispel et al., in prep). A full timing solution will be given by Thornton et al. (in prep), who found it to be in an approximately circular binary orbit of period 6.7 days with a $\gtrsim 1.3 M_{\odot}$ companion. Since a companion of this mass could be a neutron star, we searched both the original PMPS data and our confirmation observation for another pulsar, but found none down to a flux limit of $0.16 \mathrm{mJy}$, assuming a detection significance of $6 \sigma$. Unlike previous searches of this kind (e.g. Lorimer et al., 2006), no correction for acceleration is needed, as the orbital period is substantially longer than the survey integration time (35 minutes). The FWHM of the profile from the confirmation observation (Figure 3.1) is $1.3 \mathrm{~ms}$. The estimate of the lower limit on the mean flux at 1400 $\mathrm{MHz}$ is $0.27 \mathrm{mJy}$. Further details of this pulsar will be published by Thornton et al. (in prep). 


\subsubsection{PSR J1546-59}

PSR J1546-59 has a period of $7.80 \mathrm{~ms}$ and a DM of $168.3 \mathrm{pc} \mathrm{cm}^{-3}$. The spectral S/N and $\chi^{2}$ of the profile from the discovery observation, reported by seek and prepfold, are 9.4 and 3.4, respectively. The inferred distance from the NE2001 model is $3.3 \mathrm{kpc}$. It was confirmed on MJD 56102 with a 35 minute observation using the Parkes telescope at $1400 \mathrm{MHz}$. The FWHM of the profile from the confirmation observation (Figure 3.1) is $670 \mu \mathrm{s}$. The estimate of the lower limit on the mean flux at $1400 \mathrm{MHz}$ is $0.20 \mathrm{mJy}$. As with PSRs J0922-52 and J1147-66, further observations are needed for timing and determining its physical characteristics.

\subsubsection{PSR J1725-3853}

PSR J1725-3853 has a period of $4.79 \mathrm{~ms}$ and a DM of $158.2 \mathrm{pc} \mathrm{cm}^{-3}$. The spectral $\mathrm{S} / \mathrm{N}$ and $\chi^{2}$ of the profile from the discovery observation, reported by seek and prepfold, are 10.2 and 4.6, respectively. The inferred distance from the NE2001 model is $2.8 \mathrm{kpc}$. Both the confirmation observation on MJD 55660 and timing observations were done with the 100-m Robert C. Byrd Green Bank Telescope (GBT) at $820 \mathrm{MHz}$, with one timing observation at $1500 \mathrm{MHz}$. The observational parameters are listed in Table 3.1. All observations were taking using GUPPI, the Green Bank Ultimate Pulsar Processing Instrument (DuPlain et al., 2008), which is built from reconfigurable off-the-shelf hardware and software available from CASPER (Collaboration for Astronomy Signal Processing and Electronics Research; Parsons et al., 2009). GUPPI samples data with 8-bit precision over bandwidths as large as 
$800 \mathrm{MHz}$, and is capable of recording all four Stokes parameters. The observations taken on MJDs 55825 and 56031 were done in a gridding format (see, e.g., Morris et al., 2002), where we took four observations around the position of the pulsar in order to better constrain the position, which greatly facilitated the timing analysis described below.

The GBT data were initially optimized by a fine search in period and dispersion measure to produce integrated pulse profiles. Using a simple Gaussian template, we extracted times-of-arrival (TOAs) from each profile via the Fourier-domain template matching algorithm (Taylor, 1992) as implemented in the get_toa.py routine in the PRESTO package. The Gaussian template for the $820 \mathrm{MHz}$ observations was made from the composite $820 \mathrm{MHz}$ profile, and the template for the $1500 \mathrm{MHz}$ observation was made from the one observation at that frequency. Gaussian templates were used because a template made from the composite profile underestimated the errors on the residuals by a factor of nine. In total, a set of 13 TOAs spanning 371 days were then fit to a simple isolated pulsar timing model using the TEMPO analysis package $^{4}$. Following a number of iterations, we were able to converge on a timing model in which the TOAs are fit by an isolated pulsar with parameters listed in Table 3.2. The TOA uncertainties were multiplied by a factor of 3 to ensure a reduced $\chi^{2}$ value in the fit of unity. The root-mean-square timing model residuals were $88 \mu \mathrm{s}$. The positional uncertainty resulting from this fit is 0.05 " in declination and 1.2 " in right ascension, while the frequency derivative is only marginally significant, given the current baseline. The final fit parameters are typical for an isolated millisecond

\footnotetext{
${ }^{4}$ http: //tempo.sourceforge.net
} 
pulsar with a characteristic age of approximately 1.6 Gyr and a surface magnetic field of $5 \times 10^{8}$ Gauss (see, e.g., Lorimer, 2008).

No coincident sources were detected in any HEASARC catalogue ${ }^{5}$, and no $\gamma$ ray source was detected in 371 days of folded $\gamma$-ray photons from the Fermi Large Area Telescope (Atwood et al., 2009). The FWHM of the composite profile made from adding all of the profiles at $820 \mathrm{MHz}$ is $865 \mu \mathrm{s}$, and the FWHM of the $1500 \mathrm{MHz}$ profile is $1.2 \mathrm{~ms}$ (Figure 3.1). The estimated mean flux at $820 \mathrm{MHz}$ is $1.1 \mathrm{mJy}$. We were unable to compute a reliable flux density at $1500 \mathrm{MHz}$ given the $0.15 \mathrm{mJy}$ detection threshold of the PMPS (Manchester et al., 2001). These flux estimates have large errors, and further observations are required to reliably calculate a spectral index.

\subsubsection{PSR J1753-2822}

PSR J1753-2822 has a period of $18.62 \mathrm{~ms}$ and a DM of $298.4 \mathrm{pc} \mathrm{cm}^{-3}$. The spectral $\mathrm{S} / \mathrm{N}$ and $\chi^{2}$ of the profile from the discovery observation, reported by seek and prepfold, are 9.8 and 2.9, respectively. The inferred distance from the NE2001 model is $4.5 \mathrm{kpc}$. Both the confirmation observation on MJD 56162 and timing observations were done with the Lovell telescope at $1520 \mathrm{MHz}$. It is in an approximately circular binary orbit of period 9.3 hours with a $\gtrsim 0.05 M_{\odot}$ companion. As with J1227-6208, no correction for acceleration is needed.

In addition to the six pulsars confirmed so far, the search analysis described in Section 3.2 resulted in several dozen statistically significant candidate pulsar signals.

\footnotetext{
${ }^{5}$ http://heasarc.gsfc.nasa.gov
} 
A list of these candidates, which will be subject to follow-up observations with the GBT and Parkes, can be found at http://astro.phys.wvu.edu/pmps.

\subsection{Conclusions}

Reprocessing of the Parkes Multibeam Pulsar Survey resulted in the discovery and confirmation of six new pulsars, PSR J0922-52, PSR J1147-66, PSR J1227-6208, PSR J1546-59, PSR J1725-3853, and PSR J1753-2822. PSR J1227-6208 was independently confirmed by Einstein@Home as well as the HTRU team in their medium-latitude survey and will be presented by Thornton et al. (in prep). Our discovery of PSRs J0922-52, J1147-66, J1227-6208, J1546-59, J1725-3853, and J1753-2822 brings the total number of millisecond pulsars found in the PMPS to 26. We present timing solutions for PSR J1725-3853 and PSR J1753-2822, and continued timing observations will allow us to further improve these solutions.

Our discovery of these six pulsars emphasizes the value of archiving pulsar search data and indicates that there are a number of as-yet-undiscovered pulsars present in the PMPS data. Given the number of pulsar candidates present, automated searches are the most efficient way to reduce the number of candidates to an amount that can be viewed in a reasonable amount of time. Due to the fact that they have both high DMs and short periods, many of our candidates are weak and close to the detection threshold, so there is a good chance they were not ranked highly by previous automated searches. Keith et al. (2009) found that weak pulsars and pulsars with high DMs were ranked highly by automated searches. However, 
most of these pulsars have long periods, i.e. periods on the order of hundreds of milliseconds. As the ratio of DM to period increases, the detected pulse profile is significantly broadened and begins to look more sinusoidal. These candidates are harder to select via ranking systems. We note that Eatough et al. (2010) found that artificial neural networks have difficulty detecting short period pulsars, with their neural network detecting only $50 \%$ of pulsars with periods less than $10 \mathrm{~ms}$. In our search strategy, every single candidate is being inspected by eye. In many of the earlier analyses of the PMPS data (e.g. Manchester et al. 2001), the candidates were also viewed by eye and it is not clear why these were not found earlier. Perhaps they were simply missed due to human fatigue. In the upcoming year, we hope to follow up and confirm many of our candidates. Along with the re-analysis of the PMPS survey data presented here, and the ongoing search by Einstein@Home, we expect the sample of millisecond pulsars found in the PMPS to increase further. 

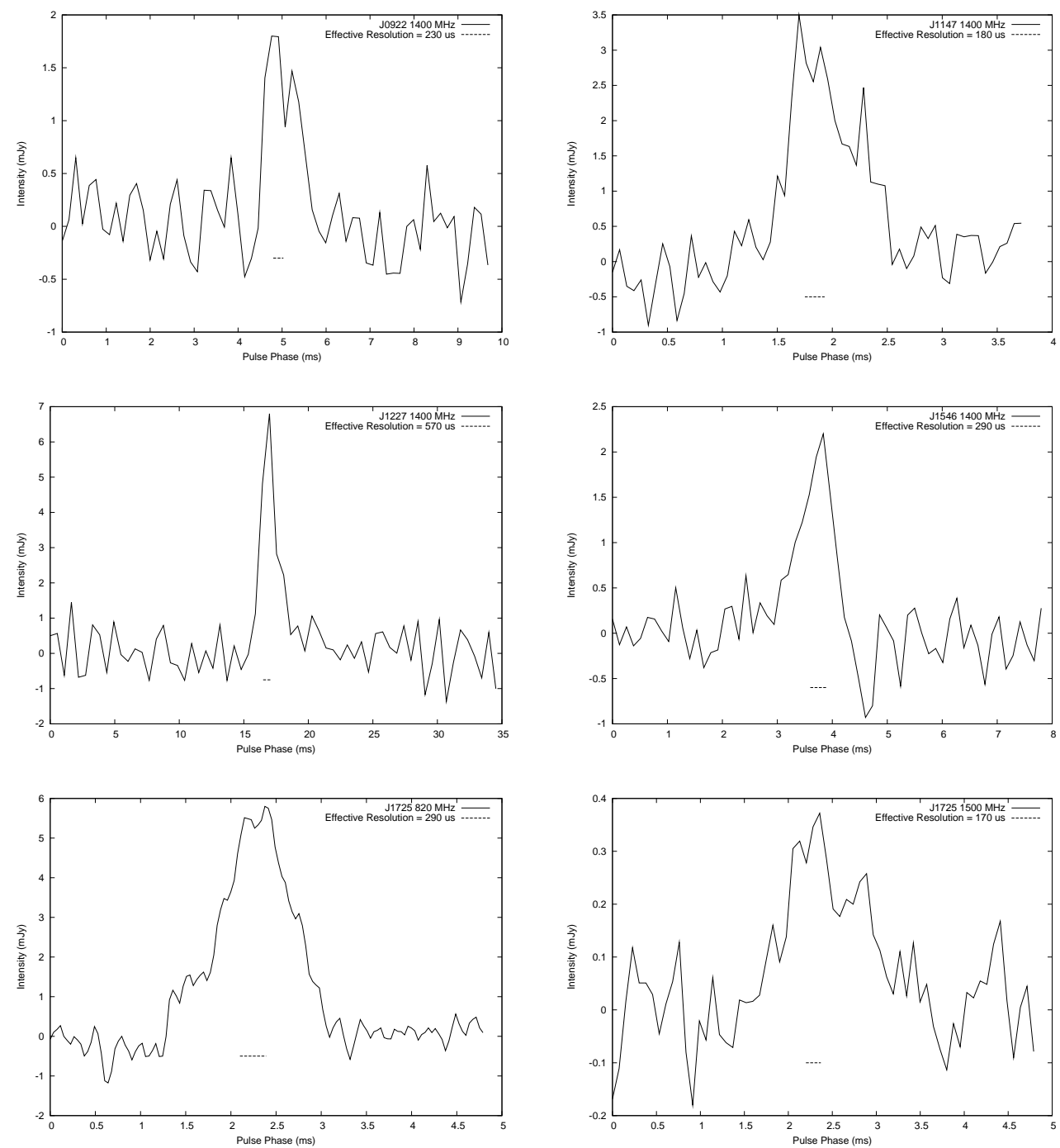

Figure 3.1: Upper Left: Folded profile from the 35 minute confirmation observation of PSR J0922-52 on MJD 56102 at $1400 \mathrm{MHz}$. The effective resolution of the profile (given by $t_{\text {eff }}=\sqrt{t_{\text {samp }}^{2}+t_{\text {scatt }}^{2}+t_{D M}^{2}}$, where $t_{\text {samp }}$ is the sampling time, $t_{\text {scatt }}$ is the scattering time from the NE2001 model (Cordes \& Lazio, 2002), and $t_{D M}$ is the DM smearing across a single channel) is given in the plot and is shown by the bar beneath the profile. Upper Right: Folded profile from the 20 minute confirmation observation of PSR J1147-66 on MJD 56158 at $1400 \mathrm{MHz}$. Middle Left: Folded profile from the 15 minute confirmation observation of PSR J1227-6208 on MJD 55857 at 1400 MHz. Middle Right: Folded profile from the 35 minute confirmation observation of PSR J1546-59 on MJD 56102 at 1400 MHz. Lower Left: Composite profile for PSR J1725-3853 at $820 \mathrm{MHz}$, with a total integration time of 113 minutes. Lower Right: Folded profile from the 15 minute $1500 \mathrm{MHz}$ observation of PSR J1725-3853 on MJD 55876. 
Table 3.1: Observational parameters for the confirmation and timing observations of PSR J1725-3853.

\begin{tabular}{cccc}
\hline MJD & Frequency $(\mathrm{MHz})$ & Bandwidth $(\mathrm{MHz})$ & Sampling Time $(\mu \mathrm{s})$ \\
\hline 55660 & 820 & 200 & 81.92 \\
55707 & 820 & 200 & 81.92 \\
55780 & 820 & 200 & 81.92 \\
55825 & 820 & 200 & 81.92 \\
55876 & 1500 & 800 & 81.92 \\
56005 & 820 & 200 & 40.96 \\
56031 & 820 & 200 & 40.96 \\
\hline
\end{tabular}

Table 3.2: Timing and derived parameters for PSR J1725-3853. Errors quoted are twice the nominal values reported by TEMPO and reflect the uncertainties in the least significant digit.

\begin{tabular}{lc}
\hline \multicolumn{2}{c}{ Timing Parameters } \\
\hline Right Ascension (J2000) & $17: 25: 27.27(8)$ \\
Declination (J2000) & $-38: 53: 04.20(5)$ \\
Spin Period (s) & $0.004791822704(3)$ \\
Period Derivative $\left(\mathrm{s} \mathrm{s}^{-1}\right)$ & $5(3) \times 10^{-20}$ \\
Dispersion Measure (pc cm $\left.{ }^{-3}\right)$ & $158.2(7)$ \\
Reference Epoch (MJD) & 55846 \\
Number of TOAs & 13 \\
Span of Timing Data & $55660-56031$ \\
\hline \multicolumn{2}{c}{ Derived Parameters } \\
\hline Galactic Longitude (degrees) & $349.3(7)$ \\
Galactic Latitude (degrees) & $-1.8(6)$ \\
Distance (kpc) & 2.8 \\
Surface Magnetic Field (Gauss) & $5(1) \times 10^{8}$ \\
Spin Down Luminosity (ergs s $\left.{ }^{-1}\right)$ & $6(3) \times 10^{33}$ \\
Characteristic Age (yr) & $1.6(9) \times 10^{9}$ \\
820 MHz Flux Density (mJy) & 1.1 \\
Pulse FWHM at 820 MHz (ms) & 0.865 \\
Pulse FWHM at 1500 MHz (ms) & 1.2 \\
\hline
\end{tabular}


Table 3.3: Timing and derived parameters for PSR J1753-2822. Errors quoted are twice the nominal values reported by TEMPO and reflect the uncertainties in the least significant digit.

\begin{tabular}{lc}
\hline \multicolumn{2}{c}{ Timing Parameters } \\
\hline Right Ascension (J2000) & $17: 53: 57.46998208$ \\
Declination (J2000) & $-28: 14: 42.3643086$ \\
Spin Period (s) & 0.01862363136 \\
Dispersion Measure (pc cm ${ }^{-3}$ ) & 298.4 \\
Reference Epoch (MJD) & 56174 \\
Number of TOAs & 35 \\
Span of Timing Data & $56162-56330$ \\
\hline \multicolumn{1}{c}{ Binary Parameters } \\
\hline Binary Model \\
Orbital Period (hours) \\
Projected Semi-major Axis (lt-s) & 9.30426 \\
Epoch of Ascending Node (MJD) & 56174.844172400 \\
Longitude of Periastron (degrees) & $158(32)$ \\
Orbital Eccentricity & 0.00092834399 \\
Mass Function (M ${ }_{\text {odot }}$ ) & 0.0000659811 \\
\hline \multicolumn{2}{c}{ Derived Parameters } \\
\hline Minimum Companion Mass (M ${ }_{\text {odot }}$ ) & 0.05058393585741712 \\
Galactic Longitude (degrees) & 1.5331 \\
Galactic Latitude (degrees) & -1.2154 \\
Distance (kpc) & 4.5 \\
\hline
\end{tabular}




\section{Chapter 4}

\section{A Study of Single Pulses in the Parkes Multibeam Pulsar Survey}

\subsection{Introduction}

While pulsars are widely used for testing relativity (e.g., Taylor \& Weisberg, 1982; Kramer et al., 2006; Antoniadis et al., 2013) and frequently timed for use in pulsar timing arrays (e.g., Jenet et al., 2009; Ferdman et al., 2010; Hobbs et al., 2010; Manchester et al., 2013), they are not entirely understood. In particular, there are still many questions regarding their emission mechanism (e.g., Weatherall, 1998; Istomin, 2004; Petrova, 2006) and equation of state (e.g., Demorest et al., 2010; Katayama et al., 2012). In order to determine these properties, rigorous studies to constrain the properties of pulsars must be carried out.

While pulsars are most commonly detected through periodicity searches, some are strong enough that we can detect single pulses from them. By studying these single pulses, we can constrain the pulsar emission mechanism. Previous studies have shown that there seem to be two distinct categories of single pulses: normal pulses and 'giant'-pulses (see, e.g., Lundgren et al., 1995; Johnston \& Romani, 2002; Kramer et al., 2002). Although giant pulses are sometimes arbitrarily defined as pulses of more than 10 times the average pulse energy (e.g., Karuppusamy et al., 2010, 2012), a comparison of the energies of normal pulses and giant pulses nonetheless show that normal pulses follow a lognormal distribution (Cognard et al., 
1996; Cairns et al., 2001, 2004), whereas giant pulses have energies that fit a powerlaw distribution (Lundgren et al., 1995; Johnston \& Romani, 2002; Kramer et al., 2002). This suggests that the emission mechanisms for these two types of pulses are different. Knight et al. (2006) argues that the definition of a giant pulse should therefore be based on short timescale, narrow-phase emission that has power-law energy statistics. Given either definition, however, pulses with amplitudes between those of normal pulses and giant pulses have yet to be fully studied. Lundgren et al. (1995) have seen an intrinsic rollover in the energy distribution of giant pulses at low energies, suggesting that there is a gap between normal pulses and giant pulses. However, observations have not yet ruled out the possibility that the power-law distribution of giant pulses is merely a high-energy tail on a lognormal distribution of normal pulses (Karuppusamy et al., 2011). These energy distributions can provide insight into the pulsar emission mechanism, so determining their true nature is important. In order to successfully do this, we need a representative sample of single pulses from a large number of objects. The number and variety of pulsars present in pulsar surveys, like the PMPS, provides us with the opportunity to carry out such a study.

One of many ways with which we can constrain single-pulse emission is by searching for correlations between pulse strength and physical properties of these pulsars. In our analysis, we used the ratio of the maximum single-pulse $\mathrm{S} / \mathrm{N}$ to the Fast Fourier Transform $(\mathrm{FFT}) \mathrm{S} / \mathrm{N}\left(\frac{\mathrm{S} / \mathrm{N}_{\mathrm{SP}}}{\mathrm{S} / \mathrm{N}_{\mathrm{FFT}}}\right)$ as a measure of the pulse strength. Here the FFT $\mathrm{S} / \mathrm{N}$ is the $\mathrm{S} / \mathrm{N}$ of the spectral feature in the FFT corresponding to the spin period of the pulsar after appropriately summing harmonics (see Chapter 
1). In our analysis, we compare this ratio with the pulsar's spin period $(P)$, period derivative $(\dot{P})$, dispersion measure $(\mathrm{DM})$, characteristic age $\left(\tau_{\mathrm{c}}\right)$, spin-down energy loss rate $(\dot{E})$, surface magnetic field $\left(B_{\text {surf }}\right)$, and magnetic field strength at the light cylinder $\left(B_{\mathrm{LC}}\right)$ (see Chapter 1 for definitions).

Work of this kind was recently performed by Burke-Spolaor et al. (2012), who studied single pulses detected in the High Time Resolution Universe (HTRU) survey (Keith et al., 2010). They detected single pulses from 315 known pulsars and performed energy distribution fits using both lognormal and Gaussian distributions, finding that most of the pulsars fit lognormal energy distributions, with only a few favoring a Gaussian distribution. They found that some of the energy distributions had multiple peaks, which they showed to be caused by mode changes in these pulsars. These mode changes, which may be due to magnetospheric reconfigurations leading to weaker or redirected emission (Timokhin, 2010), can account for the nulls seen in some pulsars. They also found no correlation between modulation parameters and physical properties.

While their study overlaps with ours, Burke-Spolaor et al. (2012) note that their search was less sensitive to faint objects, due to the shorter integration time of the HTRU survey compared to the PMPS. This makes us more likely to find new pulsars in periodicity searches (see Chapter 3). These periodicity searches, which Burke-Spolaor et al. (2012) did not carry out, enabled us to calculate $\frac{\mathrm{S} / \mathrm{N}_{\mathrm{SP}}}{\mathrm{S} / \mathrm{N}_{\mathrm{FFT}}}$ ratio. Longer integration times also allow us to record more single pulses, putting better constraints on our amplitude distributions. Also, we searched for single pulses with widths of up to two seconds, while Burke-Spolaor et al. (2012) were only sensitive 
to pulses with widths less than 32 ms.

Here we present an analysis of single pulses from all known pulsars and RRATs detected in the PMPS. In Section 4.2 we describe the data reduction and analysis. Section 4.3 discusses all of the known pulsars from which we detected single pulses, while Section 4.4 presents the single-pulse results from the RRATs. Finally, conclusions are given in Section 4.5.

\subsection{Data Reduction}

We used freely-available software tools implemented in the sigproc software package $^{1}$ to search the PMPS data for periodic and single-pulse sources. The initial results of our periodicity search are discussed in Chapter 3. Here we focus on the search for individual pulses. We searched each beam in the survey for both periodicity candidates and single pulses out to a DM of $5000 \mathrm{pc} \mathrm{cm}^{-3}$, selecting single pulses with $\mathrm{S} / \mathrm{N}>5$ and with pulse widths of up to two seconds.

Initially, frequency channels in the data were shifted to correct for dispersion due to free electrons in the interstellar medium, a process called dedispersion. The amount the channels were shifted was based on the DM searched. To search for pulsars and single pulses, we dedispersed the data using many different DM values. The total number of DMs searched was 203 and was optimally chosen by dedisperse_all ${ }^{2}$, the program we used to dedisperse our data due to its speed and efficiency. Dedispersion of the data leads to a time series for each DM, and these

\footnotetext{
${ }^{1}$ sigproc.sourceforge.net

${ }^{2}$ http://www.github.com/swinlegion
} 
time series are then processed by seek, a program which searches for periodic and single-pulse signals from a source. The periodicity-search analysis implemented in seek is the standard Fourier-based approach (see Chapter 1) where the amplitude spectrum is subject to multiple harmonic folds, summing 2, 4, 8, and 16 harmonics. This process increases sensitivity to narrow pulses in a close-to-optimal fashion (Ransom et al., 2002). The single-pulse analysis performed by seek uses a top-hat function to match filter pulses of different widths, i.e., pulses are detected by fitting them with top-hat functions. In order to be sensitive to wider pulses, the time samples in the data are smoothed by factors of two, i.e., the data are added in pairs at each smoothing step. In our analysis, we used a total of 13 smoothings, resulting in a search for pulses with widths of up to $2^{13}$ time samples (2.048 seconds). Once all of the DMs were searched, all single pulses with $\mathrm{S} / \mathrm{N}>5$ were saved. These pulses are used to create a single-pulse plot (see Figure 4.1 for an example), which shows the arrival time of each pulse versus its DM, as well as the $\mathrm{S} / \mathrm{N}$ of each pulse.

For each pulsar in the ATNF pulsar catalogue ${ }^{3}$, we identified all beams for which the beam center was within 30 arcminutes of the pulsar. We then searched each of these beams for single pulses with $\mathrm{S} / \mathrm{N}>5$ and within $10 \%$ of the DM of the pulsar. We assumed that single pulses with a $\mathrm{DM}<1 \mathrm{pc} \mathrm{cm}^{-3}$ were radio frequency interference (RFI). To determine if single pulses at other DMs were associated with RFI, we measured the width and the arrival time of each RFI pulse. If the arrival time of a single pulse was within the pulse width of an RFI pulse around its arrival time, we compared their $\mathrm{S} / \mathrm{N}$ s. If the $\mathrm{S} / \mathrm{N}$ was higher in the RFI pulse, we considered

\footnotetext{
${ }^{3}$ http://www . atnf .csiro.au/people/pulsar/psrcat/
} 
the pulse from the known pulsar to be RFI. We performed the same reduction for the all known RRATs ${ }^{4}$. The single pulses that were not attributed to a known pulsar, RRAT, or RFI were recorded as burst candidates.

For each pulsar, we then examined candidates from a periodicity search of the closest beam(s). We kept all periodicity candidates with an FFT $\mathrm{S} / \mathrm{N}>9$. If the spin period and DM of the pulsar were within $10 \%$ of the spin period and DM of any of the candidates, we noted the pulsar as being detected in a periodicity search of that beam, and also recorded its FFT S/N. Some pulsars were detected only at harmonics of their actual periods, which we count as detections. For pulsars which were detected in a periodicity search of equidistant beams, we used the beam in which the FFT S/N was highest for our reduction, as the other beams gave similar results. We inspected the single-pulse plot from the beam by eye to determine if single pulses were visible, in which case we recorded all pulses with $\mathrm{S} / \mathrm{N}>5$ and within $10 \%$ of the DM of the pulsar. We then folded these pulses using parameters obtained from the ATNF pulsar catalogue. Pulses that were not in phase with the majority of the pulses were deemed spurious and removed.

Some single pulses were wide enough that they were detected in consecutive time samples. However, these multiple detections were from one pulse, and therefore, for the purpose of determining the number of single pulses recorded from a source, were only counted once. For every pulse recorded within a spin period, we compared it with the preceding and subsequent pulse within a spin period. If it is not the strongest of the three, the next pulse is tested. After recording the strongest time

\footnotetext{
${ }^{4}$ http: //astro.phys.wvu.edu/rratalog/
} 
sample for each pulse, we determined the number of single pulses seen in each observation and the maximum single-pulse $\mathrm{S} / \mathrm{N}$.

We also calculated the energy of every pulse in order to create pulse-energy distributions. To do this, we computed the on-pulse energy in every pulse period by integrating over a number of phase bins in an 'ON' window and subtracting the off-pulse mean. This 'ON' window differed for each pulsar, and was chosen to encompass the total folded profile, based on visual inspection. Since there was significant deviation in the number of noise bins selected when determining the 'ON' window by hand, we tested the sensitivity of the resulting amplitude distribution to the inclusion of many noise bins in the 'ON' window. We found that there was no significant change to the amplitude distributions for a wide range of noise bins included in 'ON' windows. To calculate the off-pulse mean, we first took the average of each 'OFF' window on either side on our 'ON' window. We took the difference of these averages and multiplied them by the pulse width. We then subtracted this area from our total folded profile.

\subsection{Known Pulsars}

In total, we recorded single pulses from 264 known pulsars out of the 1049 present in the survey. We based our detections on pulses from the beam closest to each of the pulsars since we could not reliably scale the energies of pulses that were substantially away from the center of the beam. For each detected pulsar, we constructed a normalized energy distribution, which we then fit with lognormal 
distributions and power-law tails. We calculated the $\frac{\mathrm{S} / \mathrm{N}_{\mathrm{SP}}}{\mathrm{S} / \mathrm{N}_{\mathrm{FFT}}}$ ratio and compared this to the physical properties of the pulsar (spin period, period derivative, DM, characteristic age, spin-down energy loss rate, surface magnetic field strength, magnetic field strength at the light cylinder) to determine if there is a correlation between them.

\subsubsection{Energy Distributions}

In order to create energy distributions for each pulsar, we used the standard method of normalizing the energy of each pulse by dividing it by the average energy over the entire observation (Ritchings, 1976; Biggs, 1992; Burke-Spolaor et al., 2012). We binned these normalized energies into differential distributions which we then fit, through least-squares fitting, with lognormal distributions and power-law tails. Our lognormal fit has the form of a scaled probability density function (PDF), given by

$$
P D F_{\log }(E)=\frac{C}{E \sigma \sqrt{2 \pi}} e^{-} \frac{(\ln E-\mu)^{2}}{2 \sigma^{2}},
$$

where $E$ is the normalized energy, $C$ is a scaling factor, $\mu$ is the mean normalized energy, and $\sigma$ is the standard deviation. Our power-law fit has the form

$$
P D F_{\text {power }}(E)=A E^{B}
$$

where $E$ is the normalized energy, $A$ is a scaling factor, and $B$ is the power-law index. These PDFs describe the relative likelihood that the pulse energies will take 
on certain values.

The best-fit parameters for each pulsar, as well as the reduced $\chi^{2}$ of the fit, the spin period, DM, number of single pulses recorded, maximum single-pulse $\mathrm{S} / \mathrm{N}$, FFT S/N (if the pulsar was detected in a periodicity search in the closest beam), and the $\frac{\mathrm{S} / \mathrm{N}_{\mathrm{SP}}}{\mathrm{S} / \mathrm{N}_{\mathrm{FFT}}}$ ratio are listed in Table 4.1 . The errors reported for the fit parameters represent one estimated standard deviation for the parameter estimates.

In pulse periods where no pulse is detected, the values of the pulse energy can be zero, positive, or negative. Since there is no physical meaning to a negative pulse energy (and these negative values are likely due to random fluctuations), we set these energies to zero. We can then identify nulling pulsars by looking for a large excess of zero-energy pulses in the pulse-energy distributions.

Some pulsars display 'bumps' on their energy distributions, and we can use these excess pulses to determine the average $\mathrm{S} / \mathrm{N}$ while the pulsar is bursting, and also to estimate how many pulses the pulsar emits in this bursting state. A comparison of the amplitude distributions of these bursting pulsars to normal pulsars can be seen in Figure 4.2. An inspection of the amplitude distributions show that roughly 17 pulsars display this bursting behavior.

As can be seen in Table 4.1, there is a wide range for estimates of $\mu$, or $\hat{\mu}$, in our lognormal fits, ranging from $\sim-11 E /\langle E\rangle$ to $\sim 3.1 E /\langle E\rangle$. A negative value of $\hat{\mu}$ is not physically possible, since no pulses have negative energy, but for the nine measurements for which we have a negative value of $\hat{\mu}$, their estimated standard deviations, or $\hat{\sigma}$, put them within $1 \hat{\sigma}$ of zero. The range of $\hat{\sigma}$ values is $-0.85 E /\langle E\rangle$ to $\sim 7 E /\langle E\rangle$. However, the large estimated standard deviation for 
the upper measurement make $\sim 1.5 E /\langle E\rangle$ a more likely upper limit. The ranges for both of these parameters are larger than those seen in previous studies, but are still consistent with those results.

For some pulsars, the tails of their distributions are well fit by a power-law. As previously stated, energy distributions of giant pulses are well represented by a power-law, whereas normal pulses are better fit by a lognormal. The power-law indices from these fits range from -13.7 to 2.2. Almost all of the pulsars in our sample which are well-fit (defined here as $0.90<\chi_{\text {red }}^{2}<1.10$ ) by a power-law tail are less well fit by a lognormal distribution. Similarly, those in our sample which are well fit by a lognormal distribution are less well fit by a power-law tail. A visual inspection of the amplitude distributions shows that $\sim 67$ pulsars have power-law tails.

\subsubsection{Comparison of $\frac{\mathrm{S} / \mathrm{N}_{\mathrm{SP}}}{\mathrm{S} / \mathrm{N}_{\mathrm{FFT}}}$ Ratio to Physical Properties}

The $\frac{\mathrm{S} / \mathrm{N}_{\mathrm{SP}}}{\mathrm{S} / \mathrm{N}_{\mathrm{FFT}}}$ ratios we calculated range from 0.01 to 1.51 . This ratio has previously been used by McLaughlin \& Cordes (2003), who also defined it as

$$
r=\frac{2 \eta}{\zeta N_{\mathrm{p}}^{1 / 2}} \frac{S_{\mathrm{max}}}{S_{\mathrm{av}}^{\prime}}
$$

where $\eta$ and $\zeta$ are pulse-shape dependent factors $(\eta \sim 1$ and $\zeta \approx 1.06$ for a Gaussian pulse), the number of pulses $N_{\mathrm{p}}=T_{\mathrm{obs}} / P, S_{\max }$ is the $\mathrm{S} / \mathrm{N}$ of the pulse peak, and $S_{\mathrm{av}}^{\prime}$ is the modified mean intensity. Deneva et al. (2009) used this definition to assign $r$ values to pulsars detected in both the PMPS and PALFA surveys, finding 
$0.005 \lesssim r \lesssim 1$ for PMPS objects, and $0.06<r<10$ for PALFA objects. Our $\frac{\mathrm{S} / \mathrm{N}_{\mathrm{SP}}}{\mathrm{S} / \mathrm{N}_{\mathrm{FFT}}}$ values fall in the range quoted by Deneva et al. (2009) for the PMPS, except for three instances where the values are slightly higher. The longer observation time of the PMPS (35 minutes) compared to PALFA (4.5 minutes) allows us to record a larger number of pulses. Based on Figure 12 from McLaughlin \& Cordes (2003), larger values of $N_{\mathrm{p}}$ increase $\frac{\mathrm{S} / \mathrm{N}_{\mathrm{SP}}}{\mathrm{S} / \mathrm{N}_{\mathrm{FFT}}}$ if the pulse amplitudes follow a power-law with an index steeper than 1.5, which the pulses from these three pulsars do (see Table $4.1)$.

We compared this $\frac{\mathrm{S} / \mathrm{N}_{\mathrm{SP}}}{\mathrm{S} / \mathrm{N}_{\mathrm{FFT}}}$ ratio for each pulsar to that pulsar's physical properties (spin period, period derivative, DM, characteristic age, spin-down energy loss rate, surface magnetic field strength, magnetic field strength at the light cylinder) to determine if there was a correlation between them. These comparisons can be seen in Figures $4.3-4.9$. The resulting correlations, including their reduced $\chi^{2}$ and significance, can be found in Table 4.2. The significances stated in the Table are the number of estimated standard deviations the parameter is away from zero. Also included in the Table are the Spearman $\rho$ rank correlation coefficients and P-values for a test of the correlation coefficient equaling zero (Press, 1986). The Spearman correlation coefficient is given by

$$
\hat{\rho}=\frac{\sum_{i=0}^{N-1}\left(R_{\mathrm{x}_{\mathrm{i}}}-\overline{R_{\mathrm{x}}}\right)\left(R_{\mathrm{y}_{\mathrm{i}}}-\overline{R_{\mathrm{y}}}\right)}{\sqrt{\sum_{i=0}^{N-1}\left(R_{\mathrm{x}_{\mathrm{i}}}-\overline{R_{\mathrm{x}}}\right)^{2}} \sqrt{\sum_{i=0}^{N-1}\left(R_{\mathrm{y}_{\mathrm{i}}}-\overline{R_{\mathrm{y}}}\right)^{2}}} .
$$

Given two variables $\mathrm{x}$ and $\mathrm{y}, \hat{\rho}$ describes how well the relationship between these 
variables can be described by a monotonic function. Each value $\mathrm{x}_{\mathrm{i}}$ and $\mathrm{y}_{\mathrm{i}}$ is given a rank, $R_{\mathrm{x}_{\mathrm{i}}}$ and $R_{\mathrm{y}_{\mathrm{i}}}$, starting from one for the smallest value and increasing in integer steps. For $\mathrm{x}_{\mathrm{i}}$ or $\mathrm{y}_{\mathrm{i}}$ with identical values, these values are given a rank which is equal to the average of their positions in ascending order. The correlation P-value varies from 0.0 to 1.0, with a small value indicating a significant correlation. As can be seen from Table 4.2, the spin period, characteristic age, spin-down energy loss rate, and the magnetic field strength at both the surface and the light cylinder have significant correlations, while the period derivative and DM show very weak correlations. We do not use the reduced $\chi^{2}$ as a determining factor here, since the large scatter in the correlations limit its usefulness. The Spearman correlation coefficient results reflect this.

Period derivative and DM do not display significant correlations with $\frac{\mathrm{S} / \mathrm{N}_{\mathrm{SP}}}{\mathrm{S} / \mathrm{N}_{\mathrm{FFT}}}$. The spin period, however, is the most significant variable. We use this fact to explain the rest of the correlations. The characteristic age, spin-down energy loss rate, and the magnetic field strength at both the surface and the light cylinder are all strongly dependent on the spin period, as shown in Lorimer \& Kramer (2004) by the following scaling laws:

$$
\tau_{\mathrm{c}} \propto \frac{P}{\dot{P}}, \quad \dot{E} \propto \frac{\dot{P}}{P^{3}}, \quad B_{\text {surf }} \propto \sqrt{P \dot{P}}, \quad B_{\mathrm{LC}} \propto \frac{\dot{P}^{1 / 2}}{P^{5 / 2}}
$$

Hence, the strong correlations seen among these properties are dominated by the correlation with spin period.

As mentioned before, Burke-Spolaor et al. (2012) recently studied similar cor- 
relations. They searched for correlations between physical properties and the $\mathrm{R}$ modulation statistic, defined by Johnston et al. (2001) as

$$
R_{\mathrm{j}}=\frac{M_{\mathrm{j}}-\mu_{\mathrm{j}}}{\sigma_{\mathrm{j}}},
$$

where $M_{\mathrm{j}}$ is the maximum value in the $\mathrm{j}^{\text {th }}$ bin, and $\mu_{\mathrm{j}}$ and $\sigma_{\mathrm{j}}$ are the mean and standard deviation of the entire observation in the $\mathrm{j}^{\text {th }}$ bin. Like us, they found several strong correlations, including those with characteristic age and spin-down energy loss rate, but these were all determined to be due to a strong anticorrelation between integrated S/N and the modulation index. Similarly, McLaughlin et al. (2009) compared cumulative probability distributions of RRATs to those of pulsars for distance, period, surface magnetic field strength, characteristic age, spin-down energy loss rate, and magnetic field at the light cylinder using a Kolmogorov-Smirnoff test (Press, 1986). They found that the period and surface magnetic field strength distributions for RRATs and pulsars are different, while the characteristic age, spin-down energy loss rate, and magnetic field at the light cylinder of RRATs have distributions that are consistent with those of normal pulsars.

\subsection{RRATs}

We based our analysis of the RRATs discovered by McLaughlin et al. (2006) on the beam they in which they were originally detected. For other RRATs, we based our analysis on the beam closest to them. We analyzed those RRATS from which we detected single pulses when examining the single-pulse plot by eye. This 
led to a total of 15 RRATs being included in our analysis.

We created energy distributions in a similar manner to the way in which they were created for known pulsars. However, since most of the RRATs were not detected in a periodicity search, an 'on' window could not be determined from the folded profile. For this reason, we took the entire spin period as our 'on' window.

The majority of the RRATs also show 'bumps' in their distribution (see Figure 4.10), similar to the 'bursting' behavior seen in some of the known pulsars (see Figure 4.2). However, most of the distributions drop nearly to zero between the peaks, resulting in two distinct distributions. For this reason, we attempted to fit two lognormal functions to each distribution. The results of these fits are listed in Table 4.3.

In general, the lognormal fits for the first distribution are generally poor, with high errors. The mean values for many of these distributions are near zero, showing that they are consistent with noise. The variations away from zero are likely due to the fact that we set all negative energies to zero, which will shift the mean in the positive direction. The fits for the second distributions, which are the actual distributions of RRAT pulses, have $\hat{\mu}$ and $\hat{\sigma}$ values that are consistent with those of the known pulsars we analyzed, although the ranges of both $\hat{\mu}$ and $\hat{\sigma}$ are smaller.

\subsection{Conclusions}

We performed single-pulse searches of the Parkes Multibeam Pulsar Survey (PMPS) out to a DM of $5000 \mathrm{pc} \mathrm{cm}^{-3}$ and widths of up to two seconds. The 35- 
minute pointings of the PMPS make it more sensitive to faint objects in periodicity searches than new surveys, e.g. the High Time Resolution Universe survey, and also allow us to detect more single pulses, putting better constraints on our amplitude distributions. We detected single pulses from 264 known pulsars, as well as 15 RRATs, in the survey. We created normalized energy distributions using the energy from each rotation of these objects and fit these with lognormal distributions and power-law tails. For known pulsars, there is a wide range in the $\hat{\mu}$ parameter of the lognormal fits $(0 E /\langle E\rangle<\hat{\mu}<3 E /\langle E\rangle)$ and a smaller range of $\hat{\sigma}$ values $(0.2$ $E /\langle E\rangle<\hat{\sigma}<1.5 E /\langle E\rangle)$. Both of these parameters have slightly wider ranges than those seen in a previous study by Burke-Spolaor et al. (2012). This could be due to the longer observation times of the PMPS, which allows us to collect more single pulses and thereby constrain the amplitude distributions better. The values of the slope of the power-law fit ranged from -13.7 to 2.2 . We also calculated the ratio of the maximum single-pulse $\mathrm{S} / \mathrm{N}$ and the $\mathrm{FFT} \mathrm{S/N}$, with resulting values from 0.01 to 1.51 . These values are consistent with estimates by Deneva et al. (2009), with a few outliers due to longer observation times. We compared these ratios to the spin period, period derivative, DM, characteristic age, spin-down energy loss rate, surface magnetic field strength, and magnetic field strength at the light cylinder of each pulsar. We found no significant correlation between the $\frac{\mathrm{S} / \mathrm{N}_{\mathrm{SP}}}{\mathrm{S} / \mathrm{N}_{\mathrm{FFT}}}$ ratio and the period derivative and DM, but found strong correlations with spin period, characteristic age, spin-down energy loss rate, and the magnetic field strength at the surface and light cylinder. The strongest correlation was between the $\frac{\mathrm{S} / \mathrm{N}_{\mathrm{SP}}}{\mathrm{S} / \mathrm{N}_{\mathrm{FFT}}}$ ratio and spin period. The other strong correlations were determined to be dominated by the 
spin period correlation. Previous studies found similar strong correlations between modulation and some physical parameters, but these were found to be caused by a strong anticorrelation between the modulation index and integrated S/N.

For most of the RRATs, their energy distributions are similar to those for our 'bursty' pulsars. However, the 'bump' is much more pronounced, enough so to be a distinct distribution. Indeed, the means of the first distribution are near zero, showing these distributions are consistent with noise. The values of $\hat{\mu}$ and $\hat{\sigma}$ for the second distributions are consistent with those from the known pulsars, but their range is smaller. 

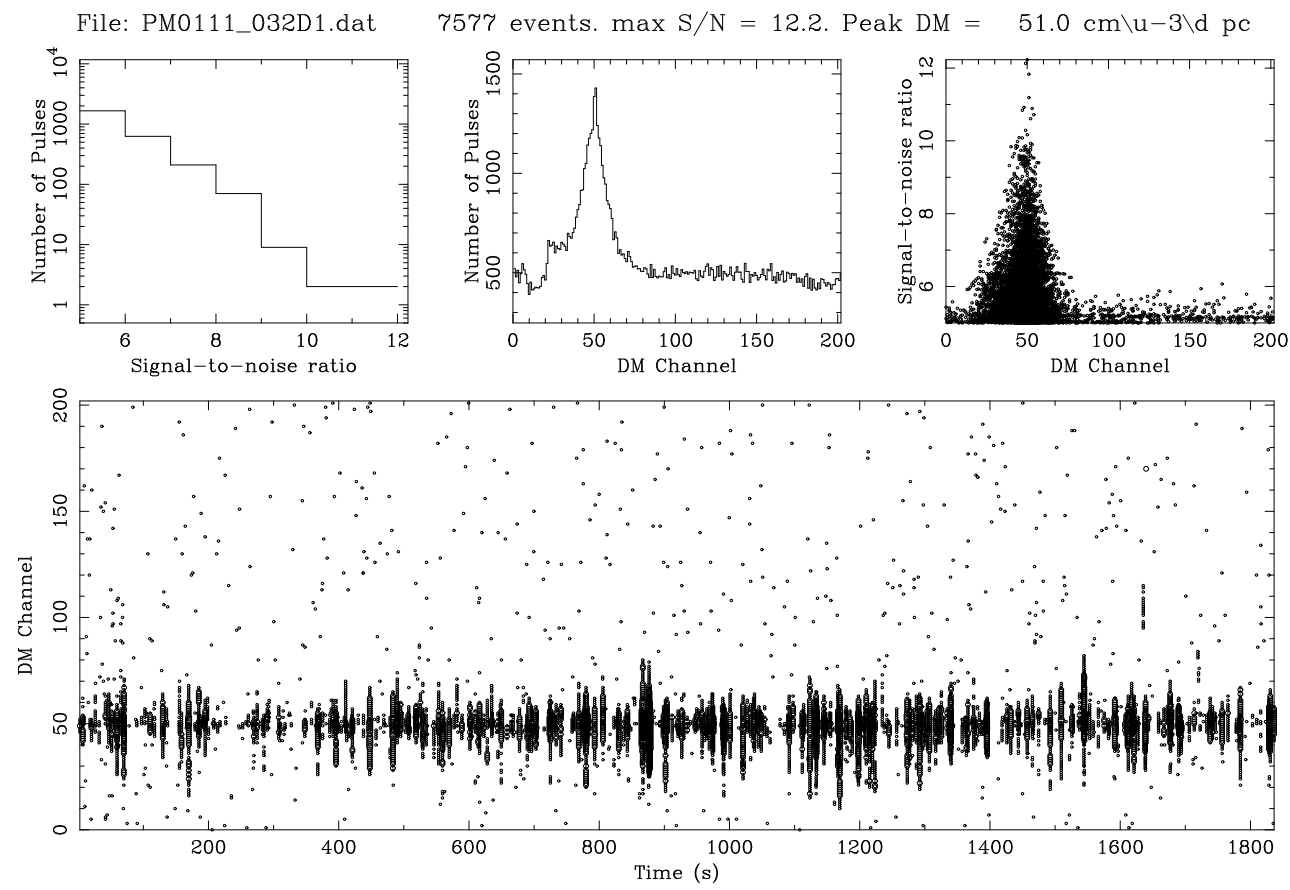

Figure 4.1: Example of our single-pulse analysis. The top plots, from left to right, show the number of pulses versus $\mathrm{S} / \mathrm{N}$, number of pulses versus DM channel, and $\mathrm{S} / \mathrm{N}$ versus DM channel. The middle and right plots show an excess of pulses with a spread of S/Ns at a DM channel of $\sim 50$ (corresponding to a DM of $\sim 60 \mathrm{pc} \mathrm{cm}^{-3}$ ). These are single pulses from PSR J0924-5814. The arrival time (relative to the start of the observation) and DM of each detected pulse can be seen in the bottom plot. The size of each point on the plot corresponds to the $\mathrm{S} / \mathrm{N}$ of the respective pulse. 

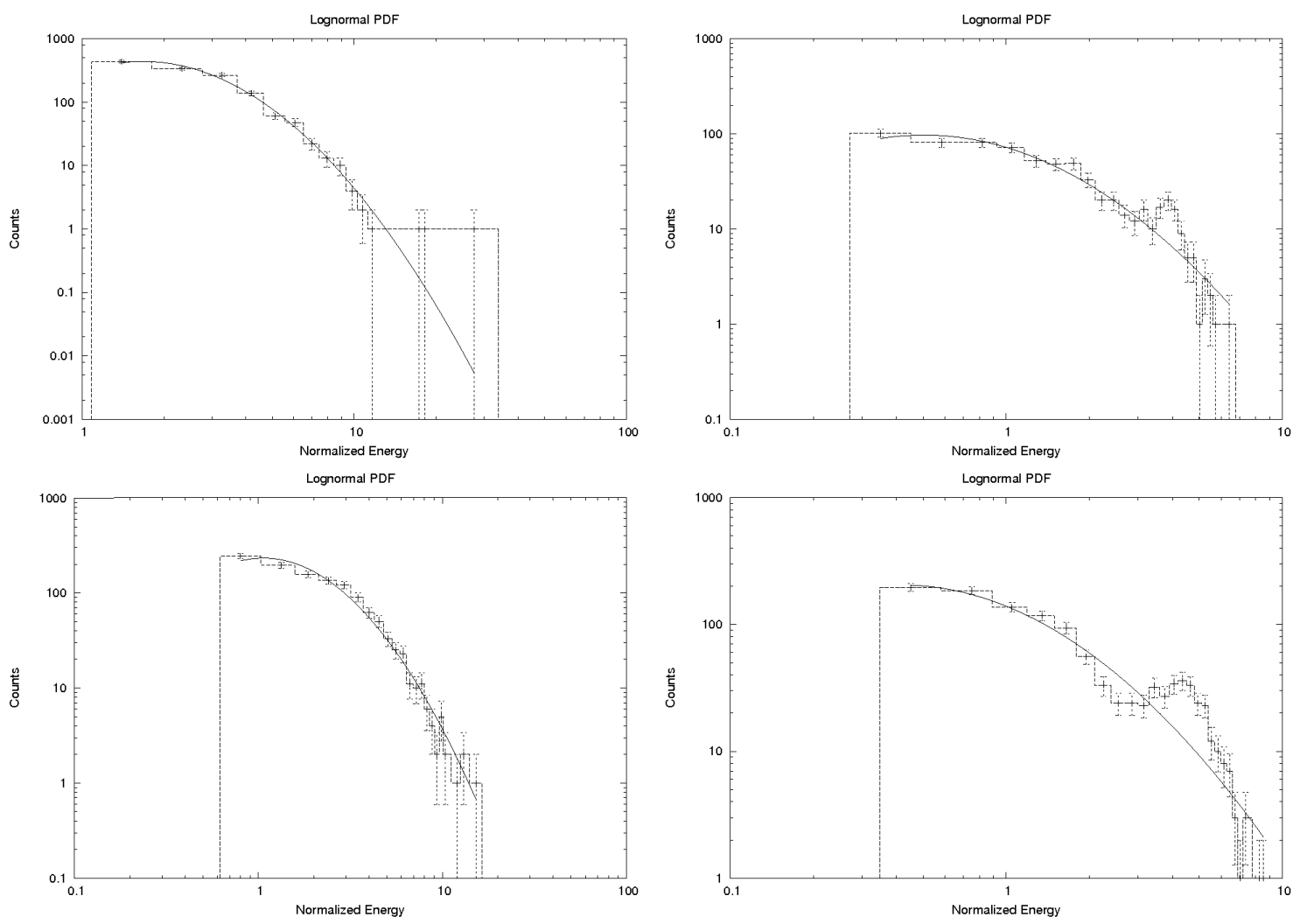

Figure 4.2: Amplitude distributions with lognormal fits. The two plots on the left are examples of normal pulsars, while the two plots on the right show 'bumps' in their distributions that we use to designate bursting pulsars. The pulsars are, clockwise from top left, PSR J0729-1836, PSR J1741-3016, PSR J1820-1346, and PSR J1038-5831. 


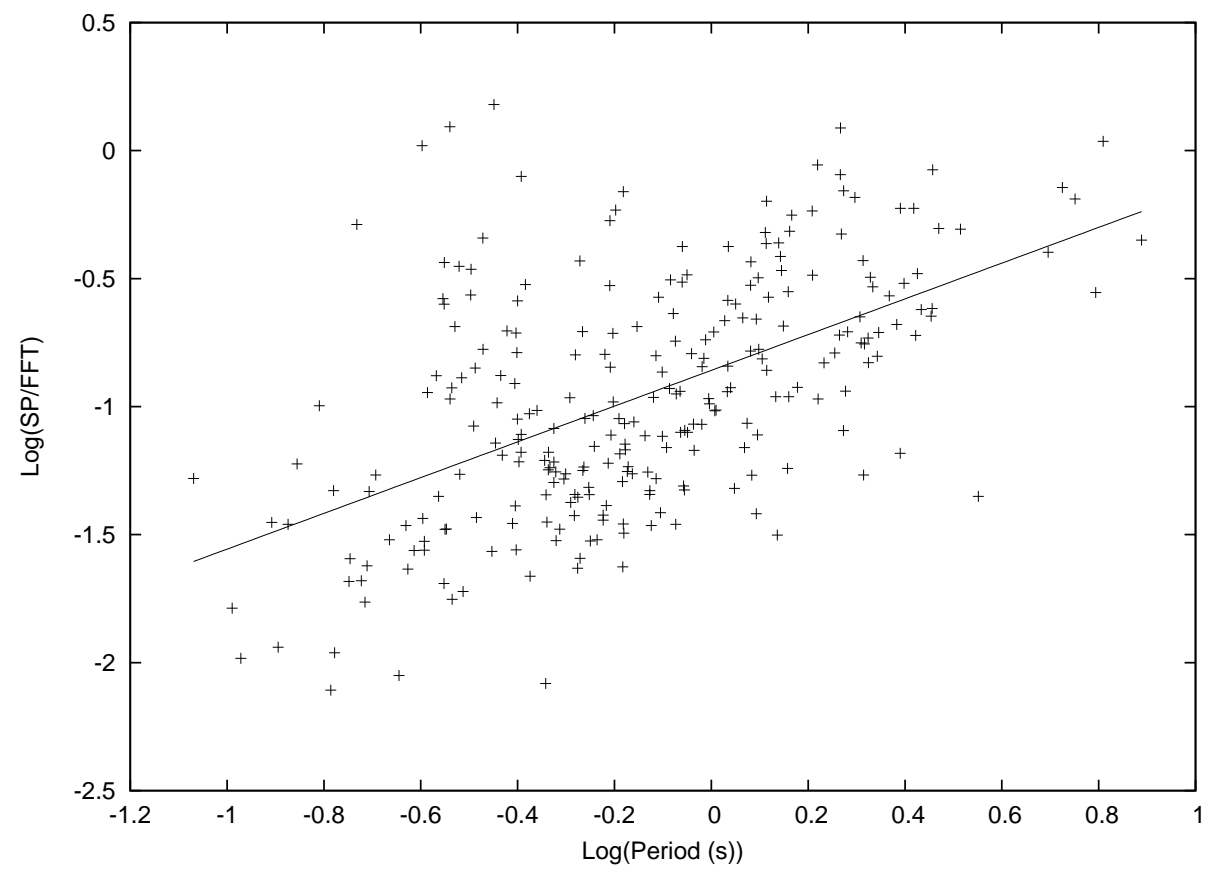

Figure 4.3: Spin period versus the ratio of maximum single-pulse S/N to FFT S/N. The best-fit line has a slope of $0.70 \pm 0.07$, which gives a correlation significance of $10 \hat{\sigma}$. The reduced $\chi^{2}$ of the fit is 0.15 . The Spearman rank correlation coefficient between the spin period and the $\frac{\mathrm{S} / \mathrm{N}_{\mathrm{SP}}}{\mathrm{S} / \mathrm{N}_{\mathrm{FFT}}}$ ratio is 0.54 , with a $\mathrm{P}$-value of $7 \times 10^{-21}$. 


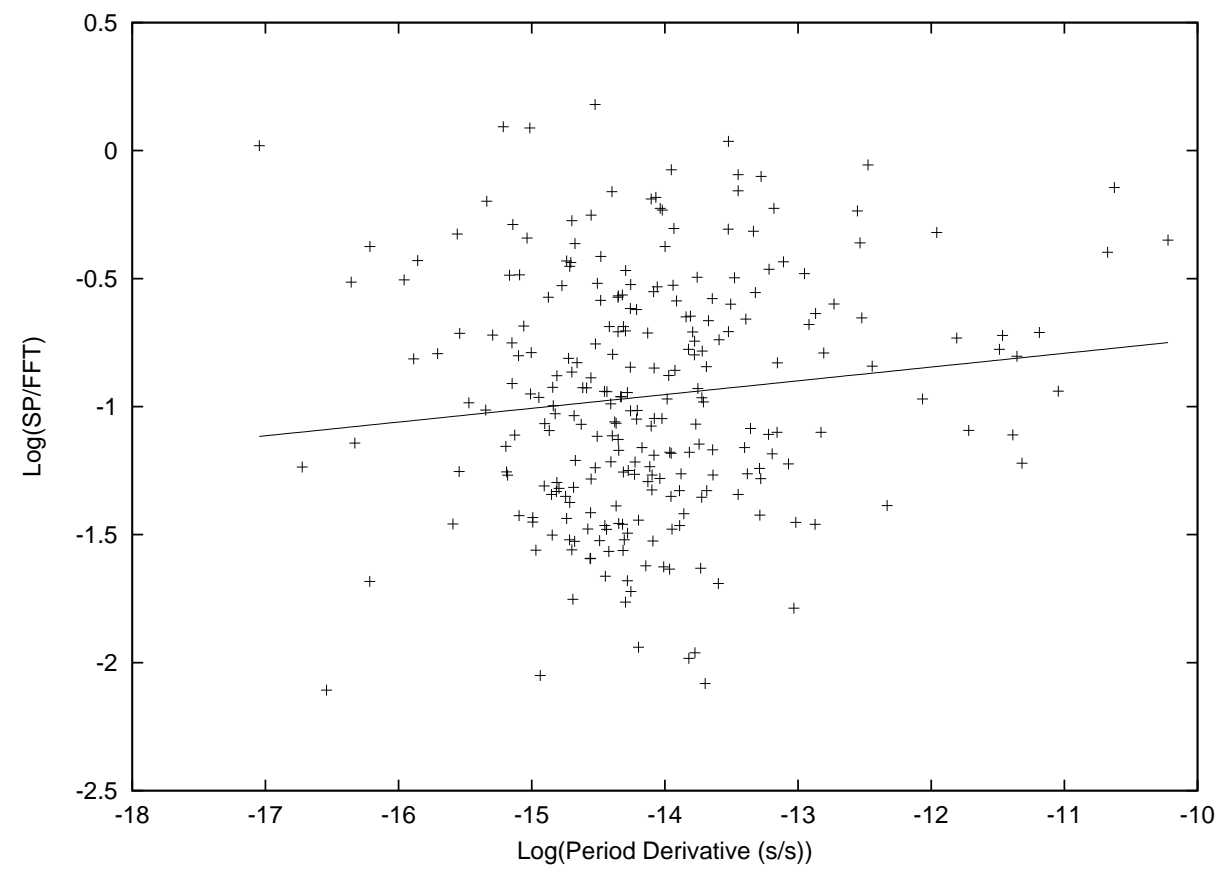

Figure 4.4: Period derivative versus the ratio of maximum single-pulse $\mathrm{S} / \mathrm{N}$ to FFT $\mathrm{S} / \mathrm{N}$. The best-fit line has a slope of $0.05 \pm 0.03$, which gives a correlation significance of $1 \hat{\sigma}$. The reduced $\chi^{2}$ of the fit is 0.22 . The Spearman rank correlation coefficient between the period derivative and the $\frac{\mathrm{S} / \mathrm{N}_{\mathrm{SP}}}{\mathrm{S} / \mathrm{N}_{\mathrm{FFT}}}$ ratio is 0.10 , with a $\mathrm{P}$-value of 0.11 . 


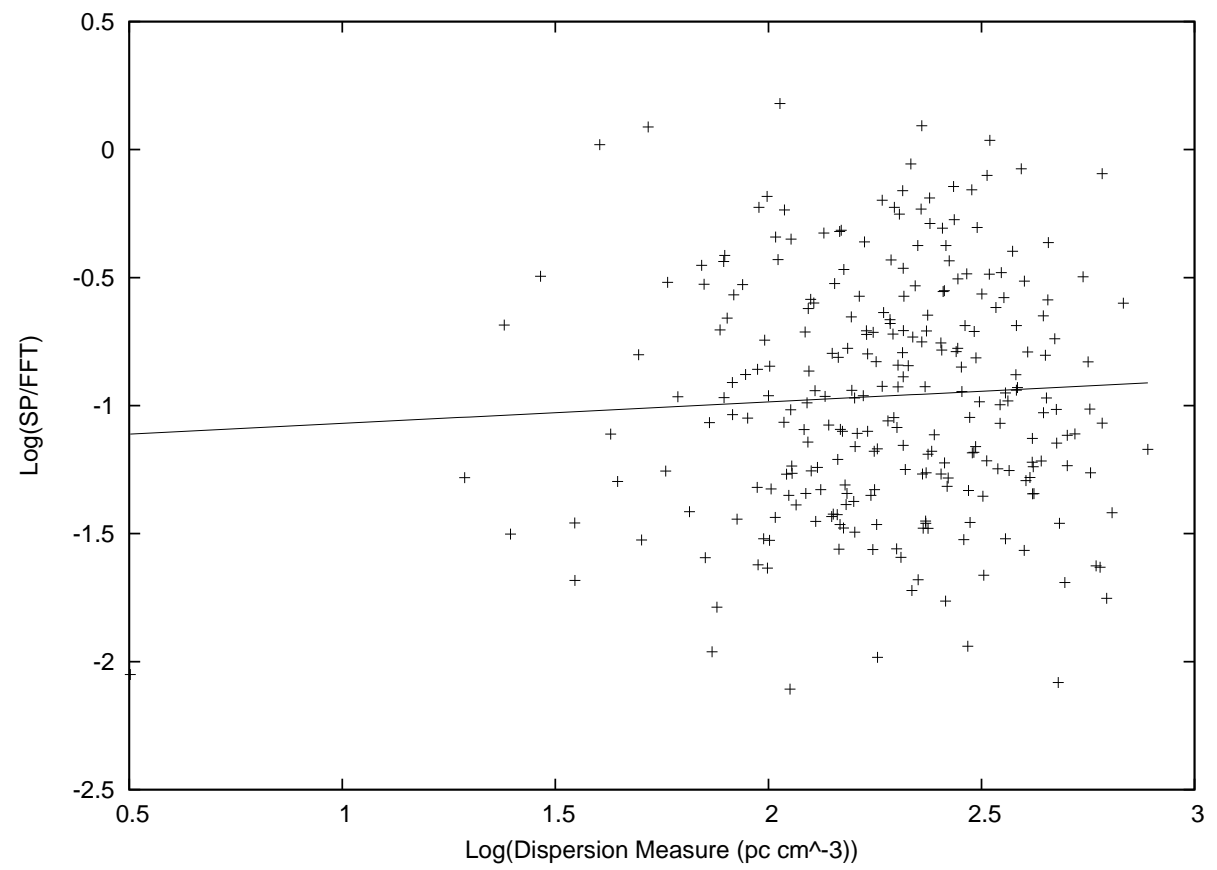

Figure 4.5: DM versus the ratio of maximum single-pulse $\mathrm{S} / \mathrm{N}$ to FFT S/N. The best-fit line has a slope of $0.08 \pm 0.09$, showing that there is no correlation. The reduced $\chi^{2}$ of the fit is 0.22 . The Spearman rank correlation coefficient between the $\mathrm{DM}$ and the $\frac{\mathrm{S} / \mathrm{N}_{\mathrm{SP}}}{\mathrm{S} / \mathrm{N}_{\mathrm{FFT}}}$ ratio is 0.03 , with a $\mathrm{P}$-value of 0.67 . 


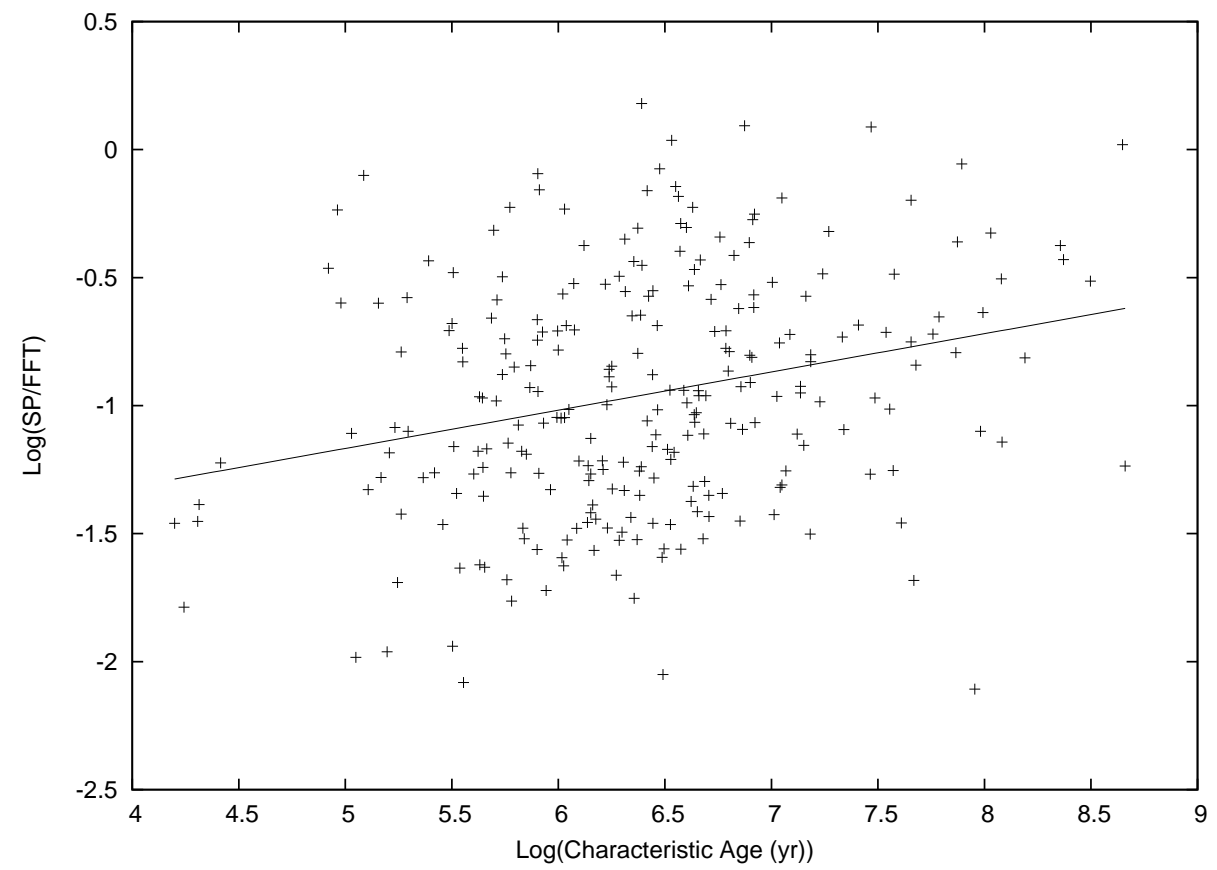

Figure 4.6: Characteristic age versus the ratio of maximum single-pulse $\mathrm{S} / \mathrm{N}$ to FFT $\mathrm{S} / \mathrm{N}$. The best-fit line has a slope of $0.15 \pm 0.03$, which gives a correlation significance of $4 \hat{\sigma}$. The reduced $\chi^{2}$ of the fit is 0.20 . The Spearman rank correlation coefficient between the characteristic age and the $\frac{\mathrm{S} / \mathrm{N}_{\mathrm{SP}}}{\mathrm{S} / \mathrm{N}_{\mathrm{FFT}}}$ ratio is 0.25 , with a $\mathrm{P}$-value of $4 \times 10^{-5}$. 


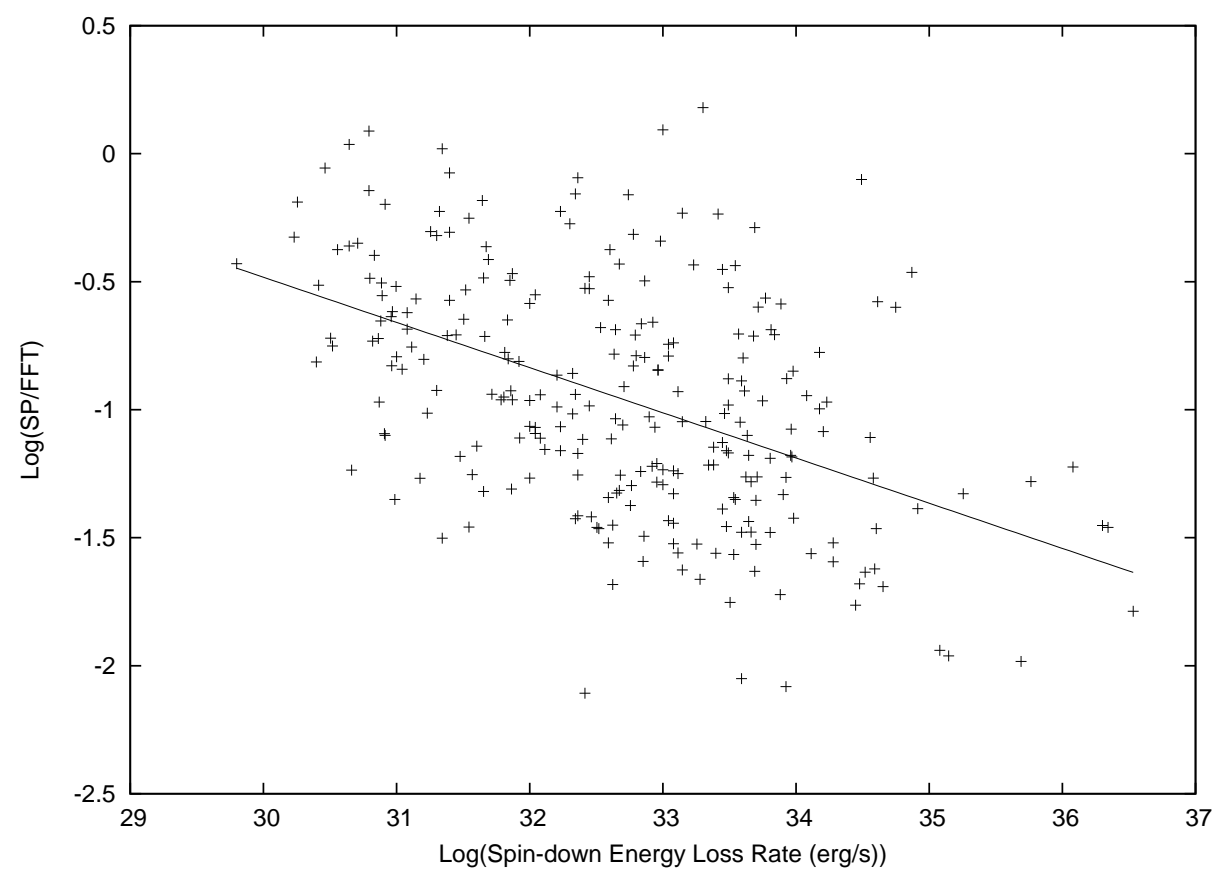

Figure 4.7: Spin-down energy loss rate versus the ratio of maximum single-pulse S/N to FFT S/N. The best-fit line has a slope of $-0.18 \pm 0.02$, which gives a correlation significance of $8 \hat{\sigma}$. The reduced $\chi^{2}$ of the fit is 0.17 . The Spearman rank correlation coefficient between the spin-down energy loss rate and the $\frac{\mathrm{S} / \mathrm{N}_{\mathrm{SP}}}{\mathrm{S} / \mathrm{N}_{\mathrm{FFT}}}$ ratio is -0.47 , with a $\mathrm{P}$-value of $1 \times 10^{-15}$. 


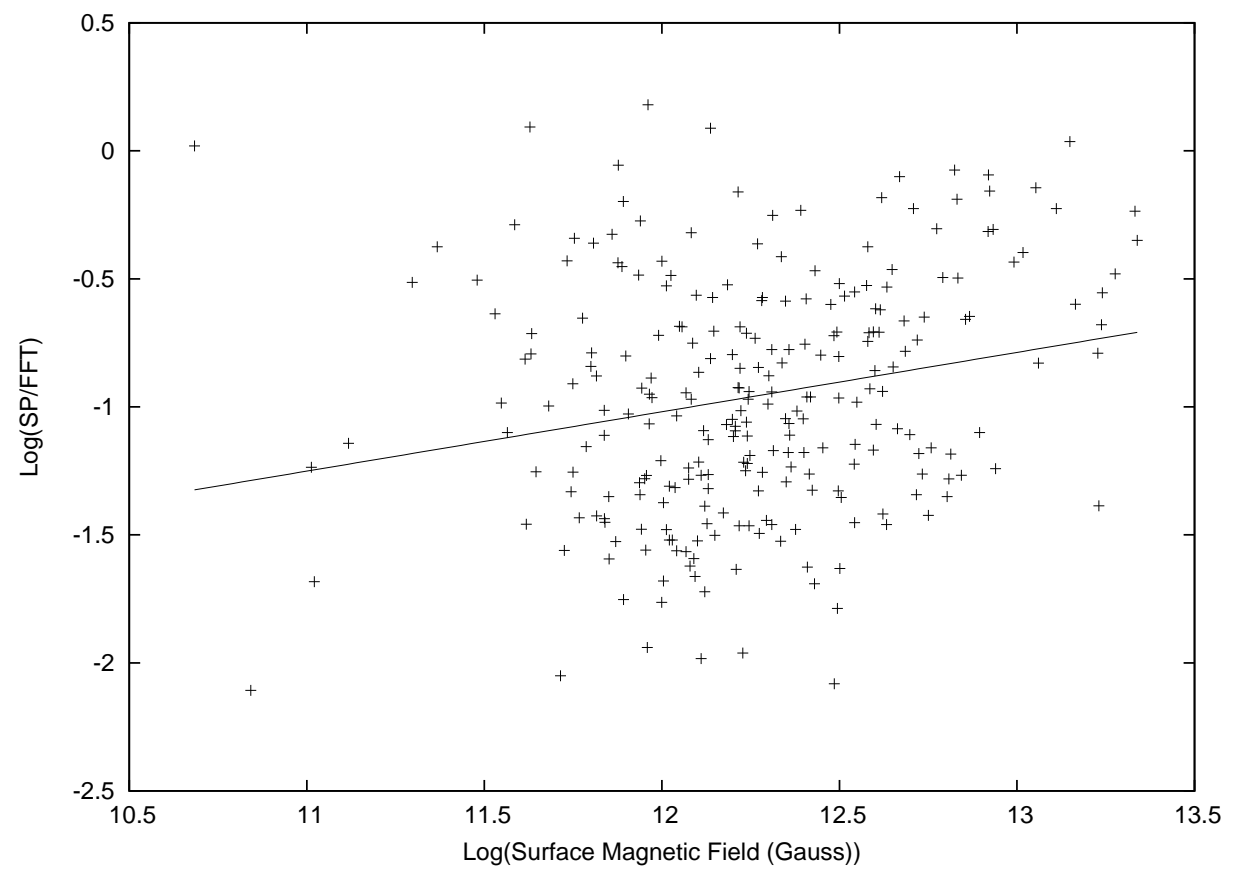

Figure 4.8: Surface magnetic field strength versus the ratio of maximum single-pulse $\mathrm{S} / \mathrm{N}$ to FFT S/N. The best-fit line has a slope of $0.23 \pm 0.06$, which gives a correlation significance of $3 \hat{\sigma}$. The reduced $\chi^{2}$ of the fit is 0.21 . The Spearman rank correlation coefficient between the surface magnetic field strength and the $\frac{\mathrm{S} / \mathrm{N}_{\mathrm{SP}}}{\mathrm{S} / \mathrm{N}_{\mathrm{FFT}}}$ ratio is 0.21 , with a $\mathrm{P}$-value of $6 \times 10^{-4}$. 


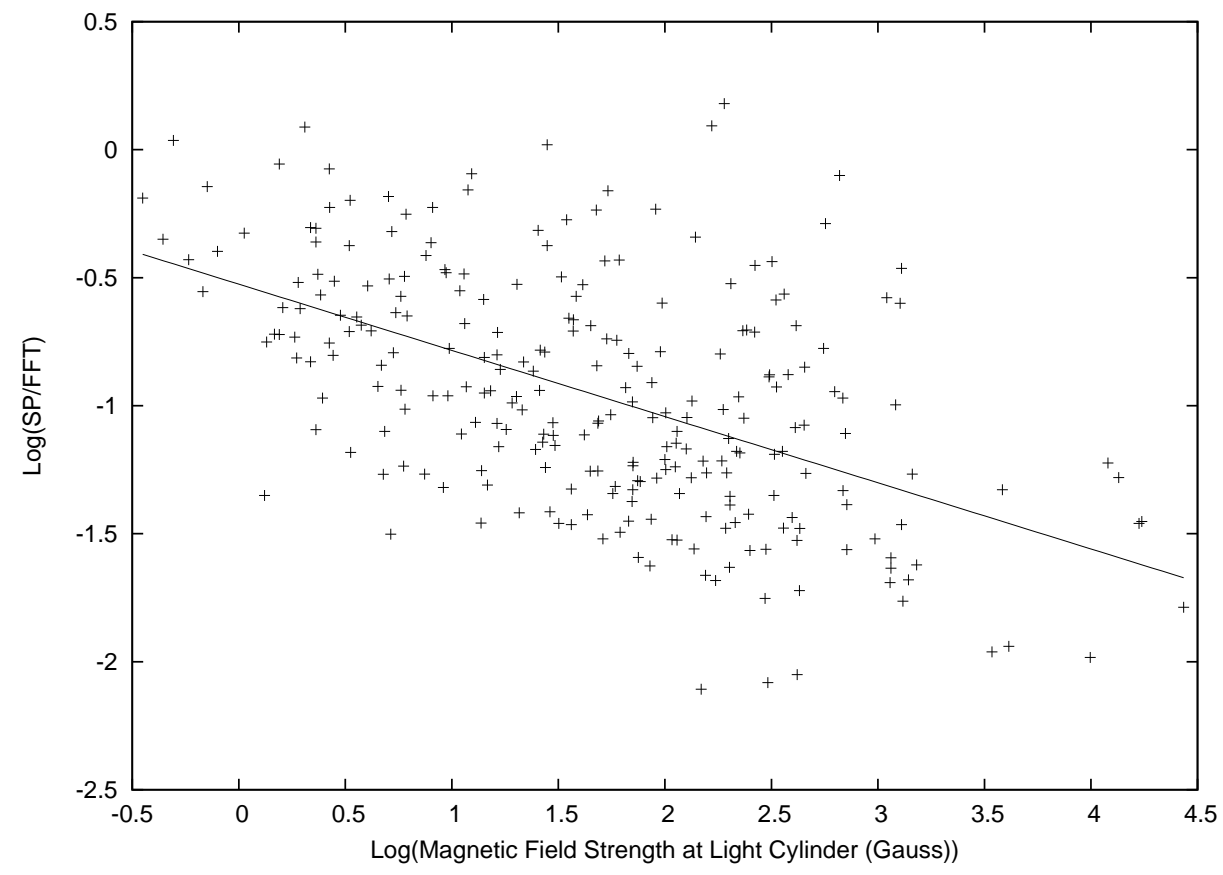

Figure 4.9: Magnetic field strength at the light cylinder versus the ratio of maximum single-pulse $\mathrm{S} / \mathrm{N}$ to FFT S/N. The best-fit line has a slope of $-0.26 \pm 0.03$, which gives a correlation significance of $9 \hat{\sigma}$. The reduced $\chi^{2}$ of the fit is 0.16 . The Spearman rank correlation coefficient between the magnetic field strength at the light cylinder and the $\frac{\mathrm{S} / \mathrm{N}_{\mathrm{SP}}}{\mathrm{S} / \mathrm{N}_{\mathrm{FFT}}}$ ratio is -0.52 , with a $\mathrm{P}$-value of $4 \times 10^{-19}$. 

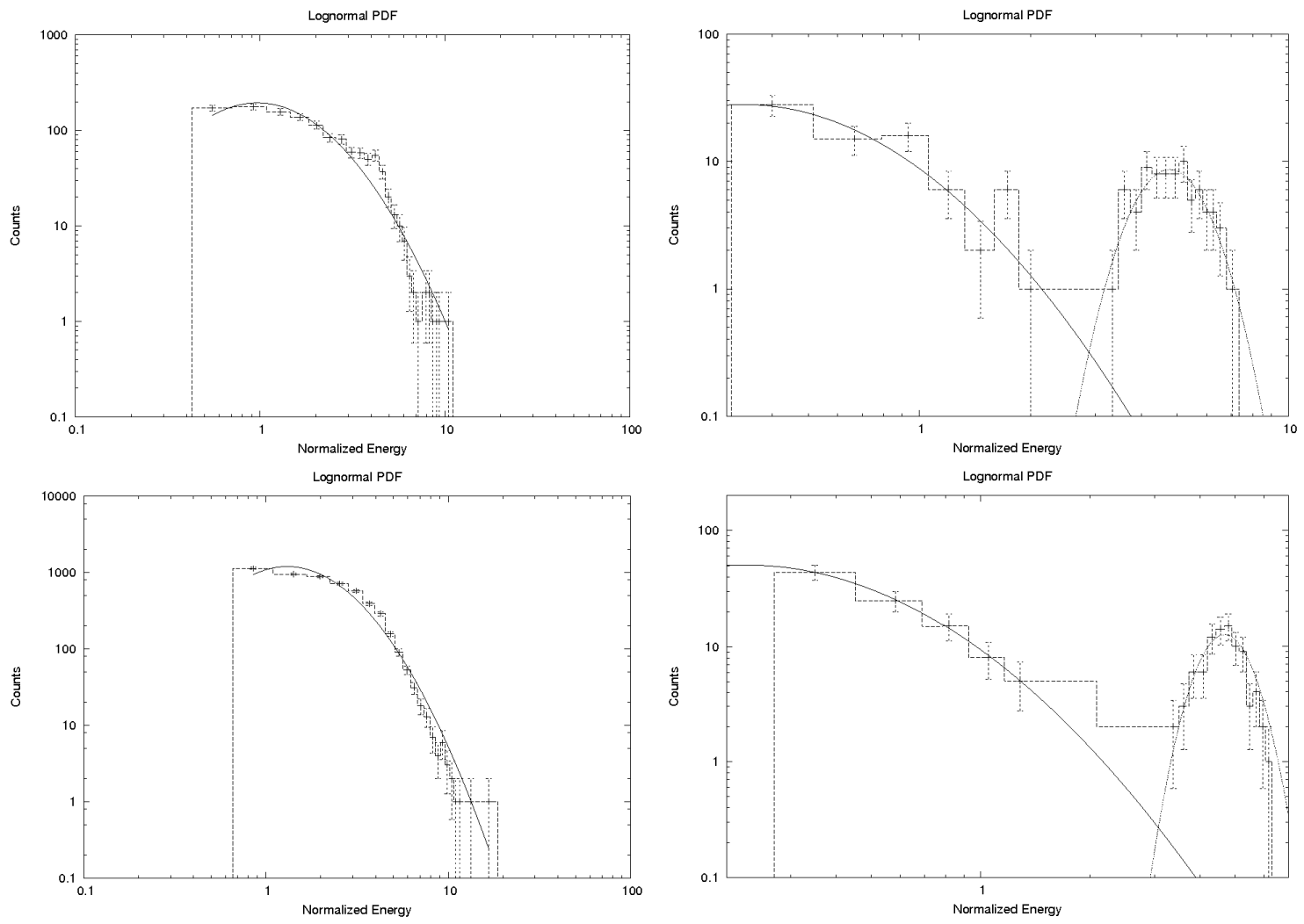

Figure 4.10: Amplitude distributions for RRATs with lognormal fits. The plots on the left are two of the three examples of RRATs which do not exhibit 'bumps' on their amplitude distributions, while the two plots on the right show these 'bumps', as do most of the RRATs we analyzed. The RRATs are, clockwise from top left, J1826-1419, J1444-6026, J1846-0257, and J1913+0904. 
Table 4.1. Single-pulse detections of known pulsars from the reprocessing of the PMPS. From left to right we list the pulsar name, spin period, DM, number of single pulses recorded out of the total number of stellar rotations during the observation, best-fit parameters from lognormal fits and the reduced $\chi^{2}$ of the fit, best-fit parameters from power-law fits and the reduced $\chi^{2}$ of the fit, peak single-pulse S/N,

FFT S/N, and the ratio of single-pulse S/N to FFT S/N. The fits were calculated through least-squares fitting of a scaled lognormal distribution, given by $\frac{C}{x \sigma \sqrt{2 \pi}} \exp \left(-\frac{(\ln (x)-\mu)^{2}}{2 \sigma^{2}}\right)$ and a power-law distribution, given by $C x^{\alpha}$. A star in the final two columns means that the pulsar was not detected in a periodicity search. Stars in the lognormal fit columns signify that we were not able to fit a lognormal function to that pulsars amplitude distribution.

\begin{tabular}{|c|c|c|c|c|c|c|c|c|c|c|c|}
\hline \multirow[t]{2}{*}{ PSR } & \multirow{2}{*}{$\begin{array}{l}\text { Period } \\
\quad(\mathrm{s})\end{array}$} & \multirow{2}{*}{$\begin{array}{c}\mathrm{DM} \\
\left(\mathrm{pc} \mathrm{cm}^{-3}\right)\end{array}$} & \multirow{2}{*}{$\begin{array}{l}\text { Number of Single } \\
\text { Pulses Detected }\end{array}$} & \multicolumn{3}{|c|}{ Lognormal } & \multicolumn{2}{|c|}{ Power Law } & \multirow{2}{*}{$\begin{array}{l}\operatorname{Max} S P \\
\mathrm{~S} / \mathrm{N}\end{array}$} & \multirow{2}{*}{$\begin{array}{l}\text { Max FFT } \\
\text { S/N }\end{array}$} & \multirow[t]{2}{*}{$\mathrm{SP} / \mathrm{FFT}$} \\
\hline & & & & $\mu(E /\langle E\rangle)$ & $\sigma(E /\langle E\rangle)$ & Reduced $\chi^{2}$ & $\alpha$ & Reduced $\chi^{2}$ & & & \\
\hline J0729-1836 & 0.510 & 61.29 & $694 / 4117$ & $0.86(0.03)$ & $0.59(0.02)$ & 1.55 & $-4.35(0.60)$ & 1.12 & 19.7 & 182.0 & 0.11 \\
\hline J0742-2822 & 0.167 & 73.78 & $4509 / 12574$ & $-0.01(0.01)$ & $0.19(0.01)$ & 52.12 & $-8.39(1.24)$ & 153.70 & 17.0 & 1556.8 & 0.01 \\
\hline J0820-4114 & 0.545 & 113.40 & $6 / 3853$ & $0.33(0.06)$ & $0.51(0.05)$ & 23.87 & $-7.72(0.70)$ & 1.72 & 7.2 & 124.0 & 0.06 \\
\hline J0828-3417 & 1.849 & 52.20 & $3 / 1135$ & $0.29(0.05)$ & $0.79(0.05)$ & 1.76 & $-3.14(1.49)$ & 1.34 & 10.3 & 8.4 & 1.23 \\
\hline J0837-4135 & 0.752 & 147.29 & $1466 / 2792$ & $0.07(0.05)$ & $0.55(0.04)$ & 12.76 & $-7.73(0.72)$ & 1.13 & 29.8 & 868.7 & 0.03 \\
\hline J0842-4851 & 0.644 & 196.85 & $200 / 3260$ & $0.54(0.05)$ & $0.79(0.04)$ & 3.98 & $-6.04(1.60)$ & 1.14 & 13.1 & 145.9 & 0.09 \\
\hline J0846-3533 & 1.116 & 94.16 & $393 / 1881$ & $0.60(0.06)$ & $0.82(0.05)$ & 2.39 & $-6.33(0.72)$ & 0.42 & 12.5 & 260.7 & 0.05 \\
\hline J0904-4246 & 0.965 & 145.80 & $133 / 2176$ & $0.59(0.05)$ & $0.84(0.04)$ & 1.95 & $-5.43(0.89)$ & 0.57 & 18.9 & 122.5 & 0.15 \\
\hline J0907-5157 & 0.254 & 103.72 & $1566 / 8267$ & $-0.04(0.04)$ & $0.55(0.03)$ & 34.48 & $-10.64(1.13)$ & 2.13 & 17.5 & 478.6 & 0.04 \\
\hline J0908-4913 & 0.107 & 180.37 & $8120 / 19626$ & $1.68(0.03)$ & $0.78(0.03)$ & 3.78 & $-4.62(1.85)$ & 1.02 & 14.3 & 1376.1 & 0.01 \\
\hline J0924-5302 & 0.746 & 152.90 & $63 / 2815$ & $0.46(0.05)$ & $0.74(0.04)$ & 4.60 & $-5.96(1.45)$ & 0.63 & 7.1 & 156.5 & 0.05 \\
\hline J0924-5814 & 0.740 & 57.40 & $200 / 2837$ & $0.90(0.04)$ & $0.80(0.04)$ & 1.70 & $-2.32(1.20)$ & 0.77 & 11.2 & 201.8 & 0.06 \\
\hline J0934-5249 & 1.445 & 100.00 & $254 / 1453$ & $0.88(0.05)$ & $0.60(0.04)$ & 2.43 & $-1.24(0.71)$ & 0.54 & 14.7 & 134.5 & 0.11 \\
\hline J0942-5552 & 0.664 & 180.20 & $589 / 3162$ & $0.37(0.05)$ & $0.74(0.04)$ & 5.01 & $-6.64(1.88)$ & 1.50 & 15.7 & 231.5 & 0.07 \\
\hline J0942-5657 & 0.808 & 159.74 & $292 / 2599$ & $0.55(0.04)$ & $0.70(0.04)$ & 2.92 & $-5.72(1.36)$ & 0.88 & 9.1 & 131.6 & 0.07 \\
\hline J0954-5430 & 0.473 & 200.30 & $26 / 4439$ & $0.65(0.04)$ & $0.72(0.03)$ & 3.44 & $-8.01(1.60)$ & 1.13 & 7.0 & 85.2 & 0.08 \\
\hline J0955-5304 & 0.862 & 156.90 & $60 / 2436$ & $0.36(0.03)$ & $0.74(0.02)$ & 1.40 & $-6.64(1.03)$ & 0.44 & 10.1 & 88.1 & 0.11 \\
\hline $\mathrm{J} 1001-5507$ & 1.437 & 130.32 & $541 / 1461$ & $0.21(0.04)$ & $0.75(0.04)$ & 1.72 & $-4.47(0.68)$ & 1.32 & 32.9 & 573.4 & 0.06 \\
\hline J1001-5559 & 1.661 & 159.30 & $43 / 1264$ & $0.38(0.04)$ & $0.87(0.04)$ & 1.04 & $-5.06(1.00)$ & 0.53 & 7.2 & 67.2 & 0.11 \\
\hline $\mathrm{J} 1001-5939$ & 7.733 & 113.00 & $95 / 271$ & $1.24(0.30)$ & $1.13(0.22)$ & 0.74 & $-0.29(1.30)$ & 0.71 & 21.4 & 47.9 & 0.45 \\
\hline $\mathrm{J} 1012-5857$ & 0.820 & 383.90 & $162 / 2560$ & $0.38(0.03)$ & $0.74(0.03)$ & 1.90 & $-4.46(0.72)$ & 0.22 & 11.2 & 95.2 & 0.12 \\
\hline J1020-5921 & 1.238 & 80.00 & $27 / 1696$ & $0.59(0.05)$ & $0.88(0.05)$ & 1.34 & $-4.62(0.70)$ & 0.43 & 9.2 & 41.9 & 0.22 \\
\hline J1032-5911 & 0.464 & 418.20 & $193 / 4525$ & $1.02(0.06)$ & $0.47(0.04)$ & 4.45 & $-4.25(0.49)$ & 8.76 & 8.5 & 147.3 & 0.06 \\
\hline J1038-5831 & 0.662 & 72.74 & $267 / 3172$ & $0.67(0.04)$ & $0.78(0.03)$ & 1.54 & $-4.22(0.79)$ & 0.53 & 11.1 & 129.4 & 0.09 \\
\hline $\mathrm{J} 1042-5521$ & 1.171 & 306.50 & $162 / 1793$ & $0.63(0.04)$ & $0.73(0.04)$ & 1.47 & $0.69(0.89)$ & 0.37 & 9.2 & 132.9 & 0.07 \\
\hline $\mathrm{J} 1043-6116$ & 0.289 & 449.20 & $188 / 7266$ & $0.55(0.04)$ & $0.71(0.03)$ & 7.83 & $-6.06(1.47)$ & 1.20 & 13.5 & 126.0 & 0.11 \\
\hline J1048-5832 & 0.124 & 129.10 & $2777 / 16935$ & $1.21(0.05)$ & $0.75(0.04)$ & 9.75 & $-5.67(0.80)$ & 0.95 & 20.7 & 586.3 & 0.04 \\
\hline $\mathrm{J} 1049-5833$ & 2.202 & 446.80 & $26 / 953$ & $0.51(0.05)$ & $0.88(0.05)$ & 0.86 & $-3.46(0.68)$ & 0.65 & 6.2 & 39.4 & 0.16 \\
\hline $\mathrm{J} 1056-6258$ & 0.422 & 320.30 & $1233 / 4976$ & $-0.01(0.03)$ & $0.37(0.02)$ & 21.01 & $-9.20(0.60)$ & 1.08 & 18.8 & 864.4 & 0.02 \\
\hline $\mathrm{J} 1059-5742$ & 1.185 & 108.70 & $486 / 1772$ & $0.73(0.06)$ & $0.96(0.06)$ & 1.46 & $-3.69(0.92)$ & 0.90 & 18.2 & 211.4 & 0.09 \\
\hline $\mathrm{J} 1103-6025$ & 0.397 & 275.90 & $11 / 5289$ & $0.54(0.04)$ & $0.66(0.03)$ & 6.46 & $-7.28(0.76)$ & 0.53 & 5.9 & 36.3 & 0.16 \\
\hline
\end{tabular}


Table 4.1-Continued

\begin{tabular}{|c|c|c|c|c|c|c|c|c|c|c|c|}
\hline \multirow[t]{2}{*}{ PSR } & \multirow{2}{*}{$\begin{array}{l}\text { Period } \\
(\mathrm{s})\end{array}$} & \multirow{2}{*}{$\begin{array}{c}\mathrm{DM} \\
\left(\mathrm{pc} \mathrm{cm}^{-3}\right)\end{array}$} & \multirow{2}{*}{$\begin{array}{l}\text { Number of Single } \\
\text { Pulses Detected }\end{array}$} & \multicolumn{3}{|c|}{ Lognormal } & \multicolumn{2}{|c|}{ Power Law } & \multirow{2}{*}{$\begin{array}{c}\text { Max SP } \\
\text { S/N }\end{array}$} & \multirow{2}{*}{$\begin{array}{c}\text { Max FFT } \\
\text { S/N }\end{array}$} & \multirow[t]{2}{*}{$\mathrm{SP} / \mathrm{FFT}$} \\
\hline & & & & $\mu(E /\langle E\rangle)$ & $\sigma(E /\langle E\rangle)$ & Reduced $\chi^{2}$ & $\alpha$ & Reduced $\chi^{2}$ & & & \\
\hline J1104-6103 & 0.281 & 78.51 & $15 / 7473$ & $0.62(0.04)$ & $0.63(0.03)$ & 10.36 & $-9.42(2.45)$ & 1.39 & 8.0 & 21.9 & 0.37 \\
\hline J1107-5907 & 0.253 & 40.20 & $18 / 8300$ & $0.88(0.03)$ & $0.46(0.02)$ & 5.36 & $-9.43(2.28)$ & 1.96 & 16.1 & 15.4 & 1.05 \\
\hline J1107-6143 & 1.799 & 406.00 & $9 / 1167$ & $0.32(0.05)$ & $0.80(0.06)$ & 1.96 & $-4.56(1.10)$ & 0.88 & 6.8 & 42.0 & 0.16 \\
\hline J1110-5637 & 0.558 & 262.56 & $151 / 3763$ & $0.76(0.04)$ & $0.71(0.04)$ & 3.26 & $-6.74(0.95)$ & 0.67 & 9.1 & 188.1 & 0.05 \\
\hline J1121-5444 & 0.536 & 204.70 & $31 / 3917$ & $0.94(0.03)$ & $0.75(0.03)$ & 1.61 & $-5.62(0.66)$ & 0.42 & 5.9 & 230.9 & 0.03 \\
\hline J1133-6250 & 1.023 & 567.80 & $60 / 2052$ & $0.84(0.07)$ & $0.87(0.07)$ & 2.55 & $-2.42(0.55)$ & 0.17 & 9.0 & 92.9 & 0.10 \\
\hline J1141-6545 & 0.394 & 116.08 & $1975 / 5329$ & $0.54(0.04)$ & $0.54(0.03)$ & 5.26 & $-8.22(1.08)$ & 0.57 & 13.7 & 334.6 & 0.04 \\
\hline J1144-6146 & 0.988 & 78.70 & $17 / 2125$ & $0.45(0.10)$ & $0.55(0.07)$ & 3.55 & $-2.57(0.25)$ & 13.04 & 7.2 & 67.0 & 0.11 \\
\hline J1146-6030 & 0.273 & 111.68 & $490 / 7692$ & $0.81(0.05)$ & $0.70(0.04)$ & 8.22 & $-9.19(3.25)$ & 2.33 & 18.4 & 412.2 & 0.04 \\
\hline J1148-5725 & 3.559 & 174.00 & $18 / 590$ & $0.60(0.06)$ & $0.84(0.06)$ & 0.82 & $-1.75(0.96)$ & 0.60 & 9.5 & * & $*$ \\
\hline J1157-6224 & 0.401 & 325.20 & $456 / 5236$ & $0.41(0.04)$ & $0.60(0.03)$ & 8.21 & $-4.48(0.60)$ & 1.35 & 12.9 & 212.0 & 0.06 \\
\hline J1202-5820 & 0.453 & 145.41 & $65 / 4635$ & $0.58(0.03)$ & $0.72(0.03)$ & 2.66 & $-5.14(0.61)$ & 0.37 & 10.1 & 164.0 & 0.06 \\
\hline J1224-6407 & 0.216 & 97.47 & $1885 / 9722$ & $0.86(0.04)$ & $0.74(0.03)$ & 7.60 & $-6.42(1.86)$ & 1.40 & 19.2 & 636.1 & 0.03 \\
\hline J1225-6035 & 0.626 & 176.10 & $15 / 3354$ & $0.57(0.05)$ & $0.71(0.04)$ & 4.61 & $-8.94(1.36)$ & 0.66 & 6.3 & 32.6 & 0.19 \\
\hline J1237-6725 & 2.111 & 179.00 & $17 / 994$ & $0.58(0.05)$ & $0.84(0.05)$ & 0.99 & $-3.93(0.73)$ & 0.91 & 6.9 & 46.5 & 0.15 \\
\hline J1239-6832 & 1.302 & 94.30 & $366 / 1612$ & $0.75(0.07)$ & $0.82(0.07)$ & 2.49 & $-5.66(1.87)$ & 1.21 & 25.6 & 184.7 & 0.14 \\
\hline $\mathrm{J} 1243-6423$ & 0.388 & 297.25 & $1850 / 5412$ & $0.18(0.05)$ & $0.62(0.03)$ & 14.55 & $-7.39(0.97)$ & 0.70 & 27.4 & 784.0 & 0.03 \\
\hline J1252-6314 & 0.823 & 278.40 & $29 / 2551$ & $0.55(0.04)$ & $0.82(0.04)$ & 1.82 & $-4.52(0.62)$ & 0.61 & 9.1 & 29.1 & 0.31 \\
\hline J1253-5820 & 0.255 & 100.58 & $394 / 8235$ & * & * & * & $-3.33(0.46)$ & 63.00 & 9.4 & 315.7 & 0.03 \\
\hline J1255-6131 & 0.658 & 206.50 & $13 / 3191$ & $0.76(0.11)$ & $0.62(0.07)$ & 5.11 & $-3.09(0.38)$ & 10.68 & 8.5 & 12.3 & 0.69 \\
\hline J1306-6617 & 0.473 & 436.90 & $47 / 4439$ & $1.02(0.04)$ & $0.78(0.04)$ & 2.42 & $-5.73(0.76)$ & 0.63 & 9.0 & 148.1 & 0.06 \\
\hline J1307-6318 & 4.962 & 374.00 & $26 / 423$ & $-0.27(0.71)$ & $0.97(0.35)$ & 1.60 & $-0.21(0.77)$ & 1.01 & 11.3 & 28.2 & 0.40 \\
\hline J1314-6101 & 2.948 & 309.00 & $91 / 712$ & $0.58(0.09)$ & $0.94(0.09)$ & 1.32 & $-3.93(0.73)$ & 0.54 & 18.3 & 36.9 & 0.50 \\
\hline J1319-6105 & 0.421 & 442.20 & $73 / 4988$ & $0.61(0.04)$ & $0.70(0.03)$ & 5.45 & $-3.30(1.25)$ & 0.84 & 9.8 & 104.5 & 0.09 \\
\hline J1326-5859 & 0.478 & 287.30 & $2260 / 4393$ & $0.16(0.05)$ & $0.65(0.04)$ & 11.63 & $-7.37(0.49)$ & 0.31 & 23.4 & 780.8 & 0.03 \\
\hline J1326-6408 & 0.793 & 502.70 & $179 / 2648$ & $0.58(0.05)$ & $0.78(0.04)$ & 3.16 & $-5.19(0.88)$ & 0.42 & 9.7 & 126.7 & 0.08 \\
\hline J1326-6700 & 0.543 & 209.60 & $768 / 3867$ & $0.07(0.05)$ & $0.65(0.04)$ & 14.71 & $-9.14(1.63)$ & 1.38 & 19.9 & 353.6 & 0.06 \\
\hline $\mathrm{J} 1327-6222$ & 0.530 & 318.80 & $1626 / 3962$ & $1.92(0.05)$ & $0.88(0.05)$ & 0.96 & $-4.28(1.16)$ & 0.59 & 30.5 & 689.2 & 0.04 \\
\hline J1327-6301 & 0.196 & 294.91 & $245 / 10714$ & $0.83(0.04)$ & $0.74(0.03)$ & 7.18 & $-6.03(0.74)$ & 0.48 & 12.2 & 261.9 & 0.05 \\
\hline $\mathrm{J} 1327-6400$ & 0.281 & 680.90 & $65 / 7473$ & $0.52(0.04)$ & $0.73(0.03)$ & 8.13 & $-7.55(1.19)$ & 1.10 & 9.5 & 37.8 & 0.25 \\
\hline J1338-6204 & 1.239 & 640.30 & $68 / 1694$ & $0.31(0.05)$ & $0.53(0.05)$ & 8.64 & $-6.62(0.78)$ & 1.47 & 8.5 & 222.9 & 0.04 \\
\hline J1340-6456 & 0.379 & 76.99 & $21 / 5540$ & $0.60(0.04)$ & $0.77(0.03)$ & 4.73 & $-5.76(0.47)$ & 0.19 & 9.6 & 48.6 & 0.20 \\
\hline $\mathrm{J} 1341-6023$ & 0.627 & 364.60 & $65 / 3349$ & $0.51(0.05)$ & $0.75(0.04)$ & 4.07 & $-6.32(1.57)$ & 1.05 & 10.3 & 98.8 & 0.10 \\
\hline J1345-6115 & 1.253 & 278.10 & $22 / 1675$ & $0.68(0.10)$ & $0.95(0.10)$ & 2.92 & $-7.61(1.47)$ & 0.91 & 9.1 & 54.4 & 0.17 \\
\hline $\mathrm{J} 1349-6130$ & 0.259 & 284.60 & $10 / 8108$ & $0.55(0.05)$ & $0.70(0.03)$ & 11.32 & $-11.21(1.50)$ & 0.53 & 8.1 & 71.4 & 0.11 \\
\hline J1355-5747 & 2.039 & 229.00 & $5 / 1029$ & $0.60(0.07)$ & $0.89(0.08)$ & 1.80 & $-3.20(0.54)$ & 0.79 & 6.7 & 37.8 & 0.18 \\
\hline $\mathrm{J} 1357-62$ & 0.456 & 416.80 & $21 / 4605$ & $0.71(0.03)$ & $0.49(0.02)$ & 3.09 & $-4.83(0.38)$ & 3.83 & 7.3 & 161.4 & 0.05 \\
\hline J1359-6038 & 0.128 & 293.71 & $3121 / 16406$ & $0.49(0.04)$ & $0.66(0.03)$ & 27.13 & $-7.84(0.85)$ & 1.51 & 9.4 & 818.1 & 0.01 \\
\hline J1401-6357 & 0.843 & 98.00 & $344 / 2491$ & $0.60(0.05)$ & $0.71(0.04)$ & 3.80 & $-5.32(0.98)$ & 1.61 & 18.4 & 102.1 & 0.18 \\
\hline J1405-5641 & 0.618 & 273.00 & $5 / 3398$ & $0.49(0.05)$ & $0.79(0.05)$ & 4.73 & $-7.51(1.32)$ & 0.86 & 7.3 & 13.7 & 0.53 \\
\hline
\end{tabular}


Table 4.1-Continued

\begin{tabular}{|c|c|c|c|c|c|c|c|c|c|c|c|}
\hline \multirow[t]{2}{*}{ PSR } & \multirow{2}{*}{$\begin{array}{l}\text { Period } \\
\quad(s)\end{array}$} & \multirow{2}{*}{$\begin{array}{c}\mathrm{DM} \\
\left(\mathrm{pc} \mathrm{cm}^{-3}\right)\end{array}$} & \multirow{2}{*}{$\begin{array}{l}\text { Number of Single } \\
\text { Pulses Detected }\end{array}$} & \multicolumn{3}{|c|}{ Lognormal } & \multicolumn{2}{|c|}{ Power Law } & \multirow{2}{*}{$\begin{array}{l}\operatorname{Max} S P \\
\text { S/N }\end{array}$} & \multirow{2}{*}{$\begin{array}{l}\text { Max FFT } \\
\text { S/N }\end{array}$} & \multirow[t]{2}{*}{$\mathrm{SP} / \mathrm{FFT}$} \\
\hline & & & & $\mu(E /\langle E\rangle)$ & $\sigma(E /\langle E\rangle)$ & Reduced $\chi^{2}$ & $\alpha$ & Reduced $\chi^{2}$ & & & \\
\hline J1406-5806 & 0.288 & 229.00 & $253 / 7291$ & $0.58(0.03)$ & $0.70(0.02)$ & 3.35 & $-5.69(0.81)$ & 0.44 & 27.9 & 22.5 & 1.24 \\
\hline $\mathrm{J} 1413-6307$ & 0.395 & 121.98 & $128 / 5316$ & $0.67(0.04)$ & $0.68(0.03)$ & 4.32 & $-4.64(0.89)$ & 0.77 & 16.8 & 86.6 & 0.19 \\
\hline $\mathrm{J} 1416-6037$ & 0.296 & 289.20 & $36 / 7094$ & $0.48(0.04)$ & $0.68(0.03)$ & 9.81 & $-6.37(0.75)$ & 1.31 & 17.1 & 83.2 & 0.21 \\
\hline $\mathrm{J} 1428-5530$ & 0.570 & 82.40 & $939 / 3684$ & $0.89(0.03)$ & $0.78(0.03)$ & 1.66 & $-4.04(1.17)$ & 0.86 & 25.6 & 277.5 & 0.09 \\
\hline $\mathrm{J} 1430-6623$ & 0.785 & 65.30 & $819 / 2675$ & $0.65(0.05)$ & $0.76(0.05)$ & 3.68 & $-3.95(2.80)$ & 1.59 & 24.0 & 623.3 & 0.04 \\
\hline $\mathrm{J} 1435-5954$ & 0.473 & 44.26 & $11 / 4439$ & $0.44(0.02)$ & $0.74(0.02)$ & 1.53 & $-6.36(0.93)$ & 1.07 & 6.0 & 118.8 & 0.05 \\
\hline $\mathrm{J} 1452-6036$ & 0.155 & 349.70 & $443 / 13548$ & $0.39(0.03)$ & $0.65(0.02)$ & 10.56 & $-6.68(1.51)$ & 0.64 & 16.2 & 160.8 & 0.10 \\
\hline $\mathrm{J} 1453-6413$ & 0.179 & 71.07 & $4806 / 11731$ & $0.17(0.05)$ & $0.61(0.04)$ & 40.64 & $-8.78(0.48)$ & 0.68 & 17.5 & 686.8 & 0.03 \\
\hline $\mathrm{J} 1502-5653$ & 0.536 & 194.00 & $196 / 3917$ & $0.49(0.04)$ & $0.77(0.04)$ & 4.41 & $-6.12(1.05)$ & 0.83 & 15.3 & 41.3 & 0.37 \\
\hline $\mathrm{J} 1504-5621$ & 0.413 & 143.00 & $9 / 5084$ & $0.64(0.04)$ & $0.66(0.03)$ & 6.25 & $-7.43(1.02)$ & 1.14 & 8.0 & 26.7 & 0.30 \\
\hline $\mathrm{J} 1513-5739$ & 0.973 & 469.70 & $4 / 2158$ & * & * & * & $-1.90(0.90)$ & 0.40 & 6.4 & 35.1 & 0.18 \\
\hline $\mathrm{J} 1519-6106$ & 2.154 & 221.00 & $32 / 974$ & $0.51(0.05)$ & $0.90(0.06)$ & 1.05 & $-6.24(1.59)$ & 0.76 & 9.1 & 31.0 & 0.29 \\
\hline $\mathrm{J} 1522-5525$ & 1.390 & 79.00 & $14 / 1510$ & $0.51(0.05)$ & $0.80(0.05)$ & 2.19 & $-4.29(0.85)$ & 0.22 & 8.8 & 22.8 & 0.39 \\
\hline J1522-5829 & 0.395 & 199.90 & $39 / 5316$ & $0.46(0.03)$ & $0.74(0.02)$ & 3.05 & $-8.91(1.37)$ & 0.83 & 6.2 & 224.7 & 0.03 \\
\hline $\mathrm{J} 1525-5417$ & 1.012 & 235.00 & $32 / 2075$ & $0.53(0.04)$ & $0.77(0.03)$ & 1.71 & $-7.33(1.35)$ & 0.95 & 7.5 & 38.3 & 0.20 \\
\hline $\mathrm{J} 1529-5355$ & 0.891 & 292.00 & $3 / 2356$ & $0.61(0.07)$ & $0.81(0.06)$ & 4.46 & $-7.65(0.94)$ & 0.48 & 7.1 & 21.7 & 0.33 \\
\hline $\mathrm{J} 1534-5334$ & 1.369 & 24.82 & $556 / 1533$ & $0.58(0.06)$ & $0.84(0.05)$ & 1.96 & $-5.76(1.14)$ & 0.41 & 19.2 & 609.3 & 0.03 \\
\hline $\mathrm{J} 1536-5433$ & 0.881 & 147.50 & $79 / 2383$ & $0.86(0.09)$ & $0.80(0.06)$ & 0.62 & $-1.46(0.74)$ & 0.61 & 10.0 & 123.8 & 0.08 \\
\hline $\mathrm{J} 1537-4912$ & 0.301 & 69.70 & $44 / 6976$ & $0.60(0.04)$ & $0.66(0.03)$ & 8.86 & $-6.32(0.68)$ & 1.33 & 8.9 & 25.2 & 0.35 \\
\hline $\mathrm{J} 1539-5626$ & 0.243 & 175.88 & $476 / 8641$ & $0.97(0.05)$ & $0.78(0.05)$ & 7.63 & $-4.91(0.74)$ & 0.42 & 10.8 & 394.2 & 0.03 \\
\hline $\mathrm{J} 1542-5303$ & 1.208 & 265.70 & $60 / 1738$ & $0.54(0.05)$ & $0.94(0.06)$ & 1.09 & $-2.99(0.29)$ & 0.35 & 12.2 & 33.2 & 0.37 \\
\hline $\mathrm{J} 1544-5308$ & 0.179 & 35.16 & $105 / 11731$ & $0.61(0.04)$ & $0.67(0.03)$ & 14.31 & $-5.18(1.62)$ & 3.91 & 6.9 & 332.5 & 0.02 \\
\hline $\mathrm{J} 1548-4927$ & 0.603 & 141.20 & $200 / 3482$ & $0.52(0.04)$ & $0.80(0.04)$ & 3.61 & $-5.32(1.09)$ & 1.17 & 15.5 & 96.9 & 0.16 \\
\hline $\mathrm{J} 1558-5756$ & 1.122 & 127.80 & $96 / 1871$ & $0.48(0.05)$ & $0.81(0.05)$ & 2.24 & $-4.76(1.62)$ & 0.74 & 11.9 & 47.3 & 0.25 \\
\hline $\mathrm{J} 1559-5545$ & 0.957 & 212.90 & $60 / 2194$ & $0.52(0.06)$ & $0.79(0.05)$ & 3.64 & $-6.08(1.24)$ & 0.92 & 9.1 & 63.5 & 0.14 \\
\hline $\mathrm{J} 1600-5044$ & 0.193 & 260.56 & $3868 / 10880$ & $1.61(0.04)$ & $0.81(0.03)$ & 2.36 & $-5.16(0.84)$ & 0.59 & 19.6 & 1136.5 & 0.02 \\
\hline $\mathrm{J} 1602-5100$ & 0.864 & 170.93 & $791 / 2430$ & $0.47(0.04)$ & $0.72(0.03)$ & 2.72 & $-7.40(1.19)$ & 0.41 & 25.5 & 321.1 & 0.08 \\
\hline J1603-5657 & 0.496 & 264.07 & $17 / 4233$ & $0.52(0.04)$ & $0.76(0.04)$ & 4.03 & $-5.62(0.63)$ & 0.49 & 6.5 & 124.7 & 0.05 \\
\hline J1604-4909 & 0.327 & 140.80 & $1148 / 6422$ & $0.65(0.04)$ & $0.73(0.03)$ & 6.21 & $-6.27(1.16)$ & 0.86 & 11.8 & 320.0 & 0.04 \\
\hline J1605-5257 & 0.658 & 35.10 & $713 / 3191$ & $0.41(0.05)$ & $0.33(0.04)$ & 32.60 & $-9.63(1.41)$ & 1.01 & 15.7 & 451.2 & 0.03 \\
\hline $\mathrm{J} 1615-5444$ & 0.361 & 312.60 & $37 / 5817$ & $0.56(0.04)$ & $0.71(0.03)$ & 5.99 & $-4.86(1.15)$ & 0.52 & 8.4 & 81.2 & 0.10 \\
\hline J1615-5537 & 0.792 & 124.48 & $25 / 2651$ & $0.51(0.05)$ & $0.82(0.04)$ & 2.67 & $-4.95(1.15)$ & 1.03 & 7.5 & 55.0 & 0.14 \\
\hline J1621-5039 & 1.084 & 261.00 & $6 / 1937$ & $0.79(0.04)$ & $0.56(0.03)$ & 1.13 & $-0.65(0.45)$ & 0.28 & 7.3 & 17.3 & 0.42 \\
\hline J1624-4613 & 0.871 & 224.20 & $18 / 2411$ & $0.58(0.06)$ & $0.82(0.06)$ & 3.59 & $-4.61(0.67)$ & 1.30 & 15.1 & * & * \\
\hline $\mathrm{J} 1626-4537$ & 0.370 & 237.00 & $85 / 5675$ & $0.51(0.04)$ & $0.71(0.03)$ & 6.06 & $-5.70(1.05)$ & 0.73 & 7.3 & 113.0 & 0.06 \\
\hline $\mathrm{J} 1632-4621$ & 1.709 & 562.90 & $53 / 1228$ & $-1.39(4.53)$ & $1.30(1.06)$ & 1.11 & $-2.63(0.16)$ & 0.95 & 7.2 & 48.6 & 0.15 \\
\hline $\mathrm{J} 1633-4453$ & 0.437 & 474.10 & $450 / 4805$ & $0.69(0.04)$ & $0.72(0.03)$ & 4.33 & $-6.19(1.53)$ & 0.57 & 14.5 & 150.0 & 0.10 \\
\hline $\mathrm{J} 1633-5015$ & 0.352 & 398.41 & $456 / 5965$ & $0.49(0.05)$ & $0.72(0.05)$ & 11.94 & $-6.89(0.52)$ & 0.30 & 9.8 & 360.6 & 0.03 \\
\hline $\mathrm{J} 1644-4559$ & 0.455 & 478.80 & $1745 / 4615$ & $-0.01(0.01)$ & $0.25(0.01)$ & 14.37 & $-7.81(0.64)$ & 13.11 & 13.1 & 1581.6 & 0.01 \\
\hline $\mathrm{J} 1649-4349$ & 0.871 & 398.60 & $17 / 2411$ & $0.68(0.06)$ & $0.93(0.06)$ & 2.24 & $-4.67(0.72)$ & 0.94 & 8.0 & 26.1 & 0.31 \\
\hline
\end{tabular}


Table 4.1-Continued

\begin{tabular}{|c|c|c|c|c|c|c|c|c|c|c|c|}
\hline \multirow[t]{2}{*}{ PSR } & \multirow{2}{*}{$\begin{array}{l}\text { Period } \\
\quad(\mathrm{s})\end{array}$} & \multirow{2}{*}{$\begin{array}{c}\mathrm{DM} \\
\left(\mathrm{pc} \mathrm{cm}^{-3}\right)\end{array}$} & \multirow{2}{*}{$\begin{array}{l}\text { Number of Single } \\
\text { Pulses Detected }\end{array}$} & \multicolumn{3}{|c|}{ Lognormal } & \multicolumn{2}{|c|}{ Power Law } & \multirow{2}{*}{$\begin{array}{l}\text { Max SP } \\
\text { S/N }\end{array}$} & \multirow{2}{*}{$\begin{array}{l}\text { Max FFT } \\
\text { S/N }\end{array}$} & \multirow[t]{2}{*}{ SP/FFT } \\
\hline & & & & $\mu(E /\langle E\rangle)$ & $\sigma(E /\langle E\rangle)$ & Reduced $\chi^{2}$ & $\alpha$ & Reduced $\chi^{2}$ & & & \\
\hline J1651-4246 & 0.844 & 482.00 & $243 / 2488$ & $-0.04(0.03)$ & $0.47(0.02)$ & 7.30 & $-8.56(0.85)$ & 0.68 & 10.4 & 300.0 & 0.03 \\
\hline J1653-3838 & 0.305 & 207.20 & $393 / 6885$ & $0.47(0.04)$ & $0.77(0.04)$ & 8.44 & $-7.08(0.71)$ & 0.44 & 18.3 & 141.2 & 0.13 \\
\hline $\mathrm{J} 1653-4249$ & 0.613 & 416.10 & $76 / 3425$ & $0.78(0.04)$ & $0.78(0.03)$ & 1.76 & $-4.51(1.15)$ & 0.26 & 7.8 & 129.8 & 0.06 \\
\hline $\mathrm{J} 1654-4140$ & 1.274 & 307.00 & $9 / 1648$ & $0.57(0.06)$ & $0.86(0.06)$ & 2.09 & $-4.29(0.74)$ & 0.30 & 7.4 & 48.2 & 0.15 \\
\hline $\mathrm{J} 1700-3312$ & 1.358 & 166.97 & $275 / 1546$ & $0.44(0.05)$ & $-0.85(0.05)$ & 1.68 & $-5.55(0.88)$ & 0.98 & 17.7 & 162.0 & 0.11 \\
\hline $\mathrm{J} 1701-3726$ & 2.455 & 303.40 & $223 / 855$ & $0.67(0.11)$ & $1.02(0.12)$ & 1.74 & $-1.26(1.79)$ & 0.87 & 15.7 & 239.1 & 0.07 \\
\hline $\mathrm{J} 1703-3241$ & 1.212 & 110.31 & $519 / 1732$ & $0.72(0.05)$ & $0.83(0.05)$ & 1.49 & $-3.72(0.56)$ & 0.23 & 18.2 & 337.2 & 0.05 \\
\hline $\mathrm{J} 1703-4851$ & 1.396 & 150.29 & $26 / 1504$ & $0.44(0.06)$ & $0.81(0.05)$ & 2.60 & $-5.97(1.44)$ & 1.08 & 12.1 & 35.6 & 0.34 \\
\hline $\mathrm{J} 1705-3423$ & 0.255 & 146.36 & $252 / 8235$ & $1.34(0.23)$ & $0.30(0.41)$ & 47.35 & $-0.35(0.42)$ & 0.26 & 8.8 & 320.0 & 0.03 \\
\hline $\mathrm{J} 1705-3950$ & 0.319 & 207.10 & $28 / 6583$ & $0.46(0.04)$ & $0.68(0.03)$ & 6.60 & $-5.87(1.47)$ & 1.00 & 13.1 & 38.1 & 0.34 \\
\hline $\mathrm{J} 1707-4053$ & 0.581 & 360.00 & $216 / 3614$ & $0.99(0.05)$ & $0.79(0.04)$ & 2.64 & $-2.34(2.58)$ & 1.25 & 10.2 & 338.1 & 0.03 \\
\hline $\mathrm{J} 1708-3426$ & 0.692 & 190.70 & $94 / 3034$ & $0.70(0.04)$ & $0.83(0.04)$ & 1.79 & $-4.49(0.39)$ & 0.14 & 15.2 & 174.5 & 0.09 \\
\hline $\mathrm{J} 1708-4522$ & 1.298 & 454.00 & $4 / 1617$ & $0.54(0.05)$ & $-0.84(0.05)$ & 1.81 & $-5.78(1.03)$ & 0.41 & 8.1 & 18.7 & 0.43 \\
\hline $\mathrm{J} 1709-4429$ & 0.102 & 75.69 & $1159 / 20588$ & $0.02(0.04)$ & $0.46(0.03)$ & 117.62 & $-11.67(0.83)$ & 1.69 & 9.4 & 576.2 & 0.02 \\
\hline $\mathrm{J} 1715-4034$ & 2.072 & 254.00 & $119 / 1013$ & $0.56(0.07)$ & $0.87(0.07)$ & 1.82 & $-4.33(1.37)$ & 1.65 & 15.5 & 88.2 & 0.18 \\
\hline $\mathrm{J} 1717-3425$ & 0.656 & 587.70 & $847 / 3201$ & $1.12(0.06)$ & $0.95(0.06)$ & 1.56 & $-4.22(1.24)$ & 1.01 & 10.5 & 443.7 & 0.02 \\
\hline $\mathrm{J} 1717-4043$ & 0.398 & 452.60 & $41 / 5276$ & $0.65(0.04)$ & $0.74(0.03)$ & 4.70 & $-5.10(0.71)$ & 0.64 & 9.5 & 36.7 & 0.26 \\
\hline $\mathrm{J} 1720-2933$ & 0.620 & 42.64 & $494 / 3387$ & $0.41(0.04)$ & $0.76(0.04)$ & 4.44 & $-5.05(1.56)$ & 1.74 & 13.7 & 177.1 & 0.08 \\
\hline $\mathrm{J} 1721-3532$ & 0.280 & 496.00 & $108 / 7500$ & $0.40(0.05)$ & $0.63(0.04)$ & 18.88 & $-8.20(0.64)$ & 0.30 & 7.4 & 363.0 & 0.02 \\
\hline $\mathrm{J} 1722-3207$ & 0.477 & 126.06 & $725 / 4402$ & $0.60(0.03)$ & $0.55(0.02)$ & 2.85 & $-6.26(0.78)$ & 1.32 & 19.4 & 349.1 & 0.06 \\
\hline $\mathrm{J} 1722-3632$ & 0.399 & 416.20 & $85 / 5263$ & $0.48(0.03)$ & $0.81(0.03)$ & 2.34 & $-6.38(0.81)$ & 0.90 & 7.7 & 103.4 & 0.07 \\
\hline $\mathrm{J} 1722-3712$ & 0.236 & 99.50 & $82 / 8898$ & $0.72(0.05)$ & $0.70(0.04)$ & 10.80 & $-8.94(0.70)$ & 0.22 & 6.3 & 271.6 & 0.02 \\
\hline $\mathrm{J} 1723-3659$ & 0.203 & 254.20 & $95 / 10344$ & $0.62(0.05)$ & $0.68(0.04)$ & 15.08 & $-8.67(1.29)$ & 1.24 & 9.4 & 173.9 & 0.05 \\
\hline J1725-3848 & 2.062 & 230.00 & $4 / 1018$ & $0.47(0.06)$ & $0.89(0.06)$ & 1.19 & $-4.63(0.61)$ & 0.16 & 8.9 & * & * \\
\hline $\mathrm{J} 1725-4043$ & 1.465 & 203.00 & $16 / 1433$ & $0.50(0.07)$ & $0.82(0.06)$ & 2.63 & $-5.70(0.85)$ & 0.27 & 10.3 & 18.4 & 0.56 \\
\hline $\mathrm{J} 1727-2739$ & 1.293 & 147.00 & $138 / 1624$ & $0.38(0.05)$ & $0.76(0.04)$ & 2.75 & $-7.09(1.79)$ & 0.76 & 24.4 & 51.0 & 0.48 \\
\hline $\mathrm{J} 1730-3350$ & 0.139 & 259.00 & $15 / 15107$ & $0.37(0.05)$ & $0.64(0.03)$ & 30.98 & $-8.34(1.30)$ & 0.83 & 8.1 & 135.5 & 0.06 \\
\hline $\mathrm{J} 1730-3353$ & 3.270 & 256.00 & $28 / 642$ & * & * & * & $-2.84(1.30)$ & 0.61 & 11.2 & 22.7 & 0.49 \\
\hline $\mathrm{J} 1733-3322$ & 1.246 & 524.00 & $8 / 1685$ & $0.25(0.05)$ & $0.60(0.04)$ & 4.82 & $-2.02(0.68)$ & 0.17 & 5.5 & 70.9 & 0.08 \\
\hline $\mathrm{J} 1733-3716$ & 0.338 & 153.50 & $634 / 6213$ & $0.29(0.03)$ & $0.70(0.03)$ & 6.09 & $-8.40(1.47)$ & 0.63 & 24.4 & 145.7 & 0.17 \\
\hline $\mathrm{J} 1736-2457$ & 2.642 & 170.00 & $29 / 794$ & $0.60(0.05)$ & $0.94(0.05)$ & 0.62 & $-4.10(0.79)$ & 0.33 & 7.8 & 41.1 & 0.19 \\
\hline $\mathrm{J} 1736-2843$ & 6.445 & 331.00 & $32 / 325$ & $0.36(0.14)$ & $1.05(0.17)$ & 1.10 & $-0.18(1.31)$ & 0.91 & 22.5 & 20.7 & 1.09 \\
\hline $\mathrm{J} 1737-3555$ & 0.398 & 89.41 & $39 / 5276$ & $0.62(0.02)$ & $0.70(0.02)$ & 0.94 & $-1.37(0.93)$ & 0.38 & 7.9 & 88.4 & 0.09 \\
\hline $\mathrm{J} 1738-2330$ & 1.979 & 99.30 & $26 / 1061$ & $0.51(0.06)$ & $0.92(0.07)$ & 1.29 & $-3.87(0.97)$ & 0.56 & 10.1 & 15.4 & 0.66 \\
\hline $\mathrm{J} 1738-3211$ & 0.768 & 49.59 & $163 / 2734$ & $0.58(0.05)$ & $0.70(0.04)$ & 1.08 & $-4.25(0.55)$ & 0.88 & 15.0 & 94.9 & 0.16 \\
\hline $\mathrm{J} 1739-2903$ & 0.323 & 138.56 & $306 / 6501$ & $0.55(0.04)$ & $0.74(0.03)$ & 6.51 & $-8.30(0.91)$ & 0.37 & 15.4 & 183.3 & 0.08 \\
\hline $\mathrm{J} 1739-3131$ & 0.529 & 600.10 & $222 / 3969$ & $0.13(0.03)$ & $0.48(0.02)$ & 10.71 & $-6.85(0.58)$ & 0.63 & 7.7 & 329.7 & 0.02 \\
\hline $\mathrm{J} 1740-3015$ & 0.607 & 152.15 & $1479 / 3459$ & $0.26(0.05)$ & $0.67(0.04)$ & 9.73 & $-7.99(0.84)$ & 0.38 & 23.4 & 570.0 & 0.04 \\
\hline $\mathrm{J} 1741-2733$ & 0.893 & 149.20 & $7 / 2351$ & $0.58(0.04)$ & $0.77(0.03)$ & 1.76 & $-8.10(1.04)$ & 0.58 & 6.4 & 80.7 & 0.08 \\
\hline $\mathrm{J} 1741-3016$ & 1.894 & 382.00 & $75 / 1108$ & $0.11(0.06)$ & $0.89(0.06)$ & 1.84 & $-8.24(0.96)$ & 0.57 & 9.9 & 86.1 & 0.11 \\
\hline
\end{tabular}


Table 4.1-Continued

\begin{tabular}{|c|c|c|c|c|c|c|c|c|c|c|c|}
\hline \multirow[t]{2}{*}{ PSR } & \multirow{2}{*}{$\begin{array}{l}\text { Period } \\
(\mathrm{s})\end{array}$} & \multirow{2}{*}{$\begin{array}{c}\mathrm{DM} \\
\left(\mathrm{pc} \mathrm{cm}^{-3}\right)\end{array}$} & \multirow{2}{*}{$\begin{array}{l}\text { Number of Single } \\
\text { Pulses Detected }\end{array}$} & \multicolumn{3}{|c|}{ Lognormal } & \multicolumn{2}{|c|}{ Power Law } & \multirow{2}{*}{$\begin{array}{c}\operatorname{Max} S P \\
\text { S/N }\end{array}$} & \multirow{2}{*}{$\begin{array}{c}\text { Max FFT } \\
\text { S/N }\end{array}$} & \multirow[t]{2}{*}{$\mathrm{SP} / \mathrm{FFT}$} \\
\hline & & & & $\mu(E /\langle E\rangle)$ & $\sigma(E /\langle E\rangle)$ & Reduced $\chi^{2}$ & $\alpha$ & Reduced $\chi^{2}$ & & & \\
\hline J1741-3927 & 0.512 & 158.50 & $910 / 4101$ & $1.06(0.06)$ & $0.80(0.05)$ & 3.06 & $-7.29(1.04)$ & 0.59 & 16.6 & 393.3 & 0.04 \\
\hline $\mathrm{J} 1743-3150$ & 2.415 & 193.05 & $118 / 869$ & $0.77(0.07)$ & $0.77(0.07)$ & 1.70 & $-2.07(0.81)$ & 0.53 & 18.0 & 86.0 & 0.21 \\
\hline $\mathrm{J} 1744-3130$ & 1.066 & 192.90 & $66 / 1969$ & $0.45(0.04)$ & $0.75(0.04)$ & 2.09 & $-4.25(0.76)$ & 1.09 & 12.9 & 59.6 & 0.22 \\
\hline $\mathrm{J} 1745-3040$ & 0.367 & 88.37 & $1692 / 5722$ & $0.34(0.04)$ & $0.64(0.03)$ & 11.59 & $-4.80(0.67)$ & 0.26 & 30.7 & 232.0 & 0.13 \\
\hline $\mathrm{J} 1747-2647$ & 0.500 & 570.00 & $6 / 4200$ & $0.52(0.05)$ & $0.68(0.04)$ & 5.41 & $-5.00(0.31)$ & 1.06 & 5.5 & 100.6 & 0.05 \\
\hline $\mathrm{J} 1750-2043$ & 5.639 & 239.00 & $4 / 372$ & $0.56(0.15)$ & $0.94(0.16)$ & 1.46 & $2.20(1.77)$ & 1.27 & 6.6 & 10.2 & 0.65 \\
\hline J1750-3157 & 0.910 & 206.34 & $60 / 2307$ & $0.63(0.09)$ & $0.79(0.07)$ & 5.55 & $-6.35(0.85)$ & 1.12 & 10.8 & 67.1 & 0.16 \\
\hline $\mathrm{J} 1751-3323$ & 0.548 & 296.70 & $45 / 3832$ & $0.68(0.05)$ & $0.80(0.04)$ & 3.40 & $-6.55(1.07)$ & 0.44 & 9.8 & 109.0 & 0.09 \\
\hline J1752-2806 & 0.563 & 50.37 & $1663 / 3730$ & $0.24(0.03)$ & $0.58(0.03)$ & 5.40 & $-4.98(1.09)$ & 0.73 & 24.9 & 833.2 & 0.03 \\
\hline $\mathrm{J} 1754-3510$ & 0.393 & 82.30 & $381 / 5343$ & $0.85(0.03)$ & $0.53(0.02)$ & 2.06 & $-4.12(0.34)$ & 5.23 & 14.0 & 113.7 & 0.12 \\
\hline $\mathrm{J} 1756-2225$ & 0.405 & 326.00 & $24 / 5185$ & $0.67(0.04)$ & $0.63(0.04)$ & 8.12 & $-3.33(1.28)$ & 3.97 & 15.3 & 19.3 & 0.79 \\
\hline $\mathrm{J} 1756-2435$ & 0.670 & 367.10 & $22 / 3134$ & $0.78(0.03)$ & $0.76(0.03)$ & 1.09 & $-3.19(1.24)$ & 0.61 & 7.4 & 132.6 & 0.06 \\
\hline $\mathrm{J} 1757-2223$ & 0.185 & 239.30 & $243 / 11351$ & $0.77(0.04)$ & $0.54(0.03)$ & 16.33 & $-4.67(0.47)$ & 18.59 & 26.0 & 50.5 & 0.51 \\
\hline $\mathrm{J} 1757-2421$ & 0.234 & 179.45 & $130 / 8974$ & $0.87(0.05)$ & $0.73(0.04)$ & 8.78 & $-5.98(0.69)$ & 0.53 & 10.4 & 303.1 & 0.03 \\
\hline $\mathrm{J} 1758-2540$ & 2.108 & 218.20 & $17 / 996$ & $0.11(0.08)$ & $0.91(0.09)$ & 1.57 & $-4.92(0.78)$ & 0.49 & 7.5 & 40.5 & 0.19 \\
\hline J1759-1956 & 2.844 & 236.40 & $36 / 738$ & $0.67(0.08)$ & $1.06(0.09)$ & 0.91 & $-3.36(0.83)$ & 0.89 & 8.1 & 35.9 & 0.23 \\
\hline $\mathrm{J} 1759-2205$ & 0.461 & 177.16 & $412 / 4555$ & $0.60(0.03)$ & $0.56(0.02)$ & 3.69 & $-4.85(0.20)$ & 0.76 & 11.9 & 179.3 & 0.07 \\
\hline J1759-3107 & 1.079 & 128.60 & $145 / 1946$ & $0.43(0.03)$ & $0.63(0.03)$ & 1.16 & $-1.51(0.49)$ & 0.30 & 9.3 & 81.3 & 0.11 \\
\hline J1801-2920 & 1.082 & 125.61 & $315 / 1940$ & $0.59(0.05)$ & $0.74(0.04)$ & 2.60 & $-6.56(1.36)$ & 1.16 & 21.0 & 80.8 & 0.26 \\
\hline J1803-1857 & 2.864 & 392.00 & $5 / 733$ & $0.59(0.05)$ & $0.83(0.05)$ & 0.80 & $-5.99(1.39)$ & 0.47 & 7.4 & 8.8 & 0.84 \\
\hline $\mathrm{J} 1803-2137$ & 0.134 & 233.99 & $15 / 15671$ & $0.49(0.04)$ & $0.63(0.03)$ & 23.27 & $-6.75(1.62)$ & 1.08 & 6.9 & 199.0 & 0.03 \\
\hline $\mathrm{J} 1806-1154$ & 0.523 & 122.41 & $143 / 4015$ & $0.92(0.03)$ & $0.75(0.03)$ & 1.43 & $-5.57(0.88)$ & 0.59 & 9.8 & 215.9 & 0.05 \\
\hline $\mathrm{J} 1807-0847$ & 0.164 & 112.38 & $2352 / 12804$ & $1.97(0.04)$ & $0.81(0.04)$ & 2.38 & $-5.14(1.36)$ & 1.25 & 10.5 & 1344.0 & 0.01 \\
\hline $\mathrm{J} 1808-0813$ & 0.876 & 151.27 & $34 / 2397$ & $0.51(0.04)$ & $0.74(0.04)$ & 2.99 & $-6.06(1.01)$ & 0.91 & 6.3 & 128.6 & 0.05 \\
\hline $\mathrm{J} 1808-2057$ & 0.918 & 606.80 & $23 / 2287$ & $0.77(0.05)$ & $0.75(0.04)$ & 1.15 & $-3.34(0.34)$ & 1.50 & 9.0 & 105.4 & 0.09 \\
\hline $\mathrm{J} 1808-2701$ & 2.458 & 95.00 & $8 / 854$ & $0.57(0.10)$ & $1.24(0.14)$ & 1.01 & $-5.60(1.04)$ & 0.47 & 8.5 & 14.3 & 0.59 \\
\hline J1809-2109 & 0.702 & 381.91 & $109 / 2991$ & $0.57(0.04)$ & $0.80(0.04)$ & 2.76 & $-5.86(1.37)$ & 0.72 & 13.2 & 64.3 & 0.21 \\
\hline $\mathrm{J} 1812-1718$ & 1.205 & 255.10 & $32 / 1742$ & $0.40(0.05)$ & $0.79(0.05)$ & 2.31 & $-1.49(1.52)$ & 1.68 & 8.7 & 52.8 & 0.16 \\
\hline J1814-0618 & 1.378 & 168.00 & $7 / 1523$ & $0.54(0.04)$ & $0.81(0.04)$ & 0.96 & $-3.56(0.39)$ & 0.82 & 5.8 & 13.3 & 0.44 \\
\hline J1815-1910 & 1.250 & 547.80 & $25 / 1680$ & $0.68(0.04)$ & $0.64(0.03)$ & 1.85 & $-4.49(0.62)$ & 0.95 & 7.8 & 24.5 & 0.32 \\
\hline $\mathrm{J} 1818-1422$ & 0.291 & 622.00 & $100 / 7216$ & $1.47(0.05)$ & $0.80(0.04)$ & 3.03 & $-2.90(0.78)$ & 0.45 & 7.4 & 418.7 & 0.02 \\
\hline $\mathrm{J} 1820-0427$ & 0.598 & 84.44 & $1308 / 3511$ & $0.39(0.04)$ & $0.70(0.03)$ & 4.02 & $-8.50(1.16)$ & 0.47 & 22.6 & 627.7 & 0.04 \\
\hline J1820-0509 & 0.337 & 104.00 & $68 / 6231$ & $0.54(0.04)$ & $0.70(0.03)$ & 6.57 & $-4.92(1.66)$ & 1.09 & 14.4 & 31.6 & 0.46 \\
\hline J1820-1346 & 0.921 & 776.70 & $32 / 2280$ & $0.12(0.10)$ & $1.00(0.10)$ & 4.66 & $-7.66(0.88)$ & 0.53 & 7.5 & 111.1 & 0.07 \\
\hline $\mathrm{J} 1822-0848$ & 0.835 & 186.30 & $10 / 2514$ & $0.57(0.05)$ & $0.76(0.05)$ & 3.79 & $-5.94(0.94)$ & 1.52 & 6.6 & 28.6 & 0.23 \\
\hline $\mathrm{J} 1822-2256$ & 1.874 & 121.20 & $267 / 1120$ & $0.18(0.08)$ & $0.95(0.09)$ & 2.51 & $-3.88(0.87)$ & 2.15 & 16.7 & 207.3 & 0.08 \\
\hline $\mathrm{J} 1823-0154$ & 0.760 & 135.87 & $41 / 2763$ & $0.58(0.04)$ & $0.72(0.03)$ & 2.08 & $-4.23(1.44)$ & 1.07 & 7.6 & 70.0 & 0.11 \\
\hline $\mathrm{J} 1823-1126$ & 1.846 & 607.00 & $53 / 1137$ & $0.70(0.07)$ & $0.69(0.07)$ & 1.58 & $-2.18(0.27)$ & 4.55 & 29.3 & 36.4 & 0.80 \\
\hline J1824-0127 & 2.499 & 58.00 & $10 / 840$ & $1.01(0.08)$ & $0.37(0.06)$ & 0.99 & $-3.10(0.46)$ & 4.31 & 5.7 & 18.8 & 0.30 \\
\hline J1824-1945 & 0.189 & 224.65 & $4036 / 11111$ & $0.54(0.05)$ & $0.66(0.03)$ & 17.48 & $-4.16(2.08)$ & 1.82 & 15.8 & 757.0 & 0.02 \\
\hline
\end{tabular}


Table 4.1-Continued

\begin{tabular}{|c|c|c|c|c|c|c|c|c|c|c|c|}
\hline \multirow[t]{2}{*}{ PSR } & \multirow{2}{*}{$\begin{array}{l}\text { Period } \\
\text { (s) }\end{array}$} & \multirow{2}{*}{$\begin{array}{c}\mathrm{DM} \\
\left(\mathrm{pc} \mathrm{cm}^{-3}\right)\end{array}$} & \multirow{2}{*}{$\begin{array}{l}\text { Number of Single } \\
\text { Pulses Detected }\end{array}$} & \multicolumn{3}{|c|}{ Lognormal } & \multicolumn{2}{|c|}{ Power Law } & \multirow{2}{*}{$\begin{array}{l}\operatorname{Max} S P \\
\text { S/N }\end{array}$} & \multirow{2}{*}{$\begin{array}{c}\text { Max FFT } \\
\mathrm{S} / \mathrm{N}\end{array}$} & \multirow[t]{2}{*}{ SP/FFT } \\
\hline & & & & $\mu(E /\langle E\rangle)$ & $\sigma(E /\langle E\rangle)$ & Reduced $\chi^{2}$ & $\alpha$ & Reduced $\chi^{2}$ & & & \\
\hline J1824-2233 & 1.162 & 156.50 & $79 / 1807$ & $0.58(0.05)$ & $0.96(0.05)$ & 1.04 & $-5.41(0.67)$ & 0.28 & 10.2 & 45.9 & 0.22 \\
\hline $\mathrm{J} 1824-2328$ & 1.506 & 185.00 & $21 / 1394$ & $0.47(0.06)$ & $0.89(0.06)$ & 1.89 & $-5.33(1.24)$ & 0.73 & 6.7 & 56.3 & 0.12 \\
\hline $\mathrm{J} 1825-0935$ & 0.769 & 19.38 & $778 / 2730$ & $0.18(0.05)$ & $0.71(0.04)$ & 6.44 & $-5.63(0.45)$ & 0.26 & 19.2 & 367.3 & 0.05 \\
\hline $\mathrm{J} 1825-1446$ & 0.279 & 357.00 & $263 / 7526$ & $0.68(0.04)$ & $0.70(0.03)$ & 5.50 & $-5.68(2.45)$ & 1.47 & 26.9 & 101.9 & 0.26 \\
\hline $\mathrm{J} 1827-0750$ & 0.271 & 381.00 & $179 / 7749$ & $0.70(0.03)$ & $0.73(0.03)$ & 3.71 & $-4.87(1.51)$ & 0.87 & 13.6 & 103.0 & 0.13 \\
\hline J1829-0734 & 0.318 & 316.80 & $8 / 6603$ & $0.58(0.04)$ & $0.71(0.03)$ & 5.22 & $-6.76(0.80)$ & 0.33 & 7.7 & 28.2 & 0.27 \\
\hline $\mathrm{J} 1829-1751$ & 0.307 & 217.11 & $2233 / 6840$ & $1.24(0.03)$ & $0.74(0.03)$ & 2.29 & $-4.51(1.01)$ & 0.51 & 14.5 & 764.6 & 0.02 \\
\hline $\mathrm{J} 1830-1059$ & 0.405 & 161.50 & $139 / 5185$ & $0.61(0.04)$ & $0.66(0.03)$ & 5.62 & $-6.34(1.19)$ & 0.42 & 10.6 & 136.1 & 0.08 \\
\hline J1830-1135 & 6.222 & 257.00 & $78 / 337$ & $1.02(0.25)$ & $1.27(0.21)$ & 0.71 & $-3.82(0.94)$ & 0.39 & 14.5 & 52.0 & 0.28 \\
\hline $\mathrm{J} 1831-1223$ & 2.857 & 342.00 & $113 / 735$ & $1.48(0.33)$ & $1.49(0.48)$ & 1.26 & $-1.69(0.78)$ & 0.47 & 21.0 & 86.9 & 0.24 \\
\hline $\mathrm{J} 1832-0827$ & 0.647 & 300.87 & $122 / 3245$ & $0.30(0.05)$ & $0.71(0.04)$ & 7.46 & $-7.79(1.06)$ & 0.64 & 8.7 & 133.0 & 0.07 \\
\hline $\mathrm{J} 1833-0338$ & 0.687 & 234.54 & $1058 / 3056$ & $0.60(0.04)$ & $0.60(0.03)$ & 3.96 & $-5.04(0.64)$ & 3.33 & 17.7 & 324.1 & 0.05 \\
\hline J1833-0827 & 0.085 & 411.00 & $78 / 24705$ & $0.54(0.04)$ & $0.70(0.03)$ & 33.07 & $-8.04(0.49)$ & 0.71 & 9.8 & 187.2 & 0.05 \\
\hline $\mathrm{J} 1834-1710$ & 0.358 & 123.80 & $7 / 5865$ & $0.59(0.05)$ & $0.74(0.04)$ & 7.66 & $-6.98(0.67)$ & 0.35 & 6.0 & 83.3 & 0.07 \\
\hline $\mathrm{J} 1835-1020$ & 0.302 & 113.70 & $35 / 6953$ & $0.60(0.04)$ & $0.62(0.03)$ & 9.28 & $-8.24(0.96)$ & 0.75 & 8.2 & 150.8 & 0.05 \\
\hline $\mathrm{J} 1835-1106$ & 0.166 & 132.68 & $5 / 12650$ & $0.64(0.04)$ & $0.68(0.03)$ & 16.56 & $-6.86(0.54)$ & 0.50 & 5.9 & 125.6 & 0.05 \\
\hline $\mathrm{J} 1837-0045$ & 0.617 & 86.98 & $5 / 3403$ & $0.40(0.04)$ & $0.74(0.03)$ & 4.33 & $-4.38(1.45)$ & 1.64 & 10.1 & 34.0 & 0.30 \\
\hline $\mathrm{J} 1837-1243$ & 1.876 & 300.00 & $54 / 1119$ & $0.60(0.08)$ & $0.93(0.08)$ & 1.91 & $-7.65(1.52)$ & 0.72 & 14.0 & 20.1 & 0.70 \\
\hline $\mathrm{J} 1837-1837$ & 0.618 & 100.74 & $36 / 3398$ & $0.50(0.04)$ & $0.76(0.04)$ & 3.68 & $-7.20(0.82)$ & 0.33 & 9.4 & 65.9 & 0.14 \\
\hline J1839-1238 & 1.911 & 169.80 & $22 / 1098$ & $0.55(0.06)$ & $0.85(0.06)$ & 1.79 & $-5.46(1.30)$ & 0.59 & 9.5 & 48.5 & 0.20 \\
\hline J1840-0809 & 0.956 & 349.80 & $405 / 2196$ & $0.46(0.04)$ & $0.81(0.04)$ & 2.05 & $-6.38(1.58)$ & 0.67 & 13.8 & 161.7 & 0.09 \\
\hline $\mathrm{J} 1840-0815$ & 1.096 & 233.20 & $109 / 1916$ & $0.70(0.06)$ & $0.79(0.05)$ & 2.55 & $-4.28(0.96)$ & 0.49 & 14.0 & 118.1 & 0.12 \\
\hline $\mathrm{J} 1840-0840$ & 5.310 & 272.00 & $67 / 395$ & $0.66(0.09)$ & 1.02(0.11) & 0.61 & $-3.39(0.85)$ & 0.20 & 35.0 & 48.8 & 0.72 \\
\hline $\mathrm{J} 1841-0157$ & 0.663 & 475.00 & $169 / 3167$ & $0.65(0.05)$ & $0.80(0.05)$ & 3.37 & $-7.86(1.16)$ & 0.65 & 10.1 & 141.6 & 0.07 \\
\hline J1841-0310 & 1.658 & 216.00 & $15 / 1266$ & $0.55(0.08)$ & $0.84(0.08)$ & 3.03 & $-5.32(1.12)$ & 1.81 & 9.4 & 10.7 & 0.88 \\
\hline J1842-0359 & 1.840 & 195.98 & $199 / 1141$ & $*$ & * & * & $-4.02(0.97)$ & 1.12 & 22.3 & 117.2 & 0.19 \\
\hline $\mathrm{J} 1843-0000$ & 0.880 & 101.50 & $335 / 2386$ & $1.42(0.16)$ & $0.61(0.08)$ & 0.70 & $-2.76(0.25)$ & 2.94 & 11.8 & 249.6 & 0.05 \\
\hline $\mathrm{J} 1843-0211$ & 2.028 & 441.70 & $123 / 1035$ & $0.33(0.05)$ & $0.82(0.05)$ & 1.58 & $-4.52(1.01)$ & 1.06 & 13.2 & 58.9 & 0.22 \\
\hline $\mathrm{J} 1844+00$ & 0.461 & 345.54 & $973 / 4555$ & $0.09(0.05)$ & $0.63(0.03)$ & 13.36 & $-7.89(0.60)$ & 0.13 & 19.6 & 345.8 & 0.06 \\
\hline J1844-0433 & 0.991 & 123.16 & $300 / 2119$ & $0.26(0.04)$ & $0.77(0.04)$ & 3.21 & $-6.32(0.59)$ & 0.24 & 15.7 & 153.2 & 0.10 \\
\hline $\mathrm{J} 1845-0434$ & 0.487 & 230.80 & $99 / 4312$ & $0.89(0.05)$ & $0.73(0.04)$ & 4.32 & $-6.73(1.83)$ & 0.48 & 7.6 & 229.0 & 0.03 \\
\hline $\mathrm{J} 1845-0826$ & 0.634 & 228.20 & $8 / 3312$ & $0.55(0.04)$ & $0.73(0.03)$ & 2.67 & $-6.25(0.64)$ & 0.67 & 7.2 & 12.3 & 0.59 \\
\hline $\mathrm{J} 1845-1351$ & 2.619 & 197.40 & $22 / 801$ & $0.44(0.06)$ & $0.79(0.05)$ & 1.35 & $-1.02(0.70)$ & 0.12 & 11.6 & 19.5 & 0.59 \\
\hline $\mathrm{J} 1847-0402$ & 0.598 & 141.98 & $448 / 3511$ & $0.43(0.05)$ & $0.71(0.04)$ & 6.56 & $-1.47(4.44)$ & 4.16 & 12.4 & 329.2 & 0.04 \\
\hline $\mathrm{J} 1847-0605$ & 0.778 & 207.90 & $88 / 2699$ & $0.54(0.05)$ & $0.82(0.05)$ & 3.40 & $-5.18(0.40)$ & 0.30 & 12.9 & 48.2 & 0.27 \\
\hline $\mathrm{J} 1848-0123$ & 0.659 & 159.53 & $931 / 3186$ & $0.58(0.03)$ & $0.56(0.02)$ & 1.80 & $-5.48(0.53)$ & 0.59 & 18.3 & 571.2 & 0.03 \\
\hline J1848-1150 & 1.312 & 163.40 & $40 / 1600$ & $0.69(0.07)$ & $0.69(0.06)$ & 2.41 & $-2.57(0.33)$ & 4.36 & 10.8 & 40.4 & 0.27 \\
\hline $\mathrm{J} 1849+0127$ & 0.542 & 207.30 & $11 / 3874$ & $0.55(0.04)$ & $0.76(0.03)$ & 3.10 & $-5.74(1.32)$ & 0.81 & 6.2 & 31.6 & 0.20 \\
\hline $\mathrm{J} 1849-0636$ & 1.451 & 148.17 & $217 / 1447$ & $0.61(0.08)$ & $0.88(0.08)$ & 2.89 & $-3.62(1.50)$ & 1.15 & 38.8 & 80.2 & 0.48 \\
\hline $\mathrm{J} 1850+0026$ & 1.082 & 201.40 & $15 / 1940$ & $0.45(0.04)$ & $0.81(0.04)$ & 1.69 & $-6.01(1.14)$ & 1.16 & 6.2 & 43.1 & 0.14 \\
\hline
\end{tabular}


Table 4.1-Continued

\begin{tabular}{|c|c|c|c|c|c|c|c|c|c|c|c|}
\hline \multirow[t]{2}{*}{ PSR } & \multirow{2}{*}{$\begin{array}{l}\text { Period } \\
\quad(\mathrm{s})\end{array}$} & \multirow{2}{*}{$\begin{array}{c}\mathrm{DM} \\
\left(\mathrm{pc} \mathrm{cm} \mathrm{cm}^{-3}\right)\end{array}$} & \multirow{2}{*}{$\begin{array}{l}\text { Number of Single } \\
\text { Pulses Detected }\end{array}$} & \multicolumn{3}{|c|}{ Lognormal } & \multicolumn{2}{|c|}{ Power Law } & \multirow{2}{*}{$\begin{array}{l}\operatorname{Max} S P \\
\text { S/N }\end{array}$} & \multirow{2}{*}{$\begin{array}{l}\text { Max FFT } \\
\text { S/N }\end{array}$} & \multirow[t]{2}{*}{$\mathrm{SP} / \mathrm{FFT}$} \\
\hline & & & & $\mu(E /\langle E\rangle)$ & $\sigma(E /\langle E\rangle)$ & Reduced $\chi^{2}$ & $\alpha$ & Reduced $\chi^{2}$ & & & \\
\hline $\mathrm{J} 1851-0053$ & 1.409 & 24.00 & $61 / 1490$ & $0.66(0.06)$ & $0.85(0.06)$ & 1.89 & $-3.34(0.71)$ & 0.39 & 11.0 & 53.3 & 0.21 \\
\hline $\mathrm{J} 1851+1259$ & 1.205 & 70.61 & $26 / 1742$ & $0.59(0.04)$ & $0.69(0.03)$ & 0.80 & $-3.13(0.65)$ & 0.49 & 7.3 & 24.5 & 0.30 \\
\hline $\mathrm{J} 1852-0635$ & 0.524 & 171.00 & $94 / 4007$ & $0.38(0.04)$ & $0.72(0.03)$ & 4.94 & $-5.91(0.99)$ & 1.40 & 13.4 & 84.1 & 0.16 \\
\hline $\mathrm{J} 1854+1050$ & 0.573 & 207.20 & $7 / 3664$ & $0.43(0.03)$ & $0.65(0.03)$ & 2.67 & $-5.62(0.46)$ & 0.87 & 7.3 & 104.4 & 0.07 \\
\hline $\mathrm{J} 1859+00$ & 0.560 & 420.00 & $53 / 3750$ & $0.39(0.04)$ & $0.79(0.04)$ & 3.85 & $-6.32(0.87)$ & 0.67 & 7.0 & 154.5 & 0.05 \\
\hline $\mathrm{J} 1901+0254$ & 1.300 & 185.00 & $77 / 1615$ & $0.49(0.07)$ & $1.00(0.08)$ & 1.97 & $-4.40(0.71)$ & 0.53 & 24.3 & 38.3 & 0.63 \\
\hline $\mathrm{J} 1901-0312$ & 0.356 & 106.40 & $66 / 5898$ & $0.56(0.03)$ & $0.72(0.02)$ & 2.62 & $-6.44(0.78)$ & 0.26 & 23.0 & 15.2 & 1.51 \\
\hline $\mathrm{J} 1901+0331$ & 0.655 & 402.08 & $1180 / 3206$ & $1.20(0.17)$ & $0.27(0.07)$ & 1.00 & $-5.91(0.47)$ & 1.95 & 23.1 & 453.6 & 0.05 \\
\hline $\mathrm{J} 1901+0413$ & 2.664 & 352.00 & $7 / 788$ & $0.73(0.09)$ & $0.64(0.09)$ & 2.90 & $-2.01(0.36)$ & 5.85 & 7.8 & 23.6 & 0.33 \\
\hline $\mathrm{J} 1902+0556$ & 0.747 & 177.49 & $213 / 2811$ & $0.46(0.05)$ & $0.76(0.04)$ & 4.00 & $-4.78(0.75)$ & 0.91 & 9.1 & 193.7 & 0.05 \\
\hline $\mathrm{J} 1902+0615$ & 0.674 & 502.90 & $73 / 3115$ & $0.47(0.04)$ & $0.74(0.03)$ & 2.82 & $-3.29(0.94)$ & 0.45 & 6.8 & 116.8 & 0.06 \\
\hline $\mathrm{J} 1903+0135$ & 0.729 & 245.17 & $947 / 2880$ & $0.52(0.05)$ & $0.69(0.04)$ & 4.16 & $-4.78(0.40)$ & 1.45 & 28.9 & 375.4 & 0.08 \\
\hline $\mathrm{J} 1904+1011$ & 1.857 & 135.00 & $5 / 1130$ & $0.33(0.44)$ & $1.25(0.36)$ & 2.84 & $-3.24(0.44)$ & 1.44 & 11.8 & 25.0 & 0.47 \\
\hline $\mathrm{J} 1908+0457$ & 0.847 & 360.00 & $56 / 2479$ & $0.63(0.05)$ & $0.80(0.05)$ & 2.88 & $-7.36(1.61)$ & 0.71 & 11.0 & 98.2 & 0.11 \\
\hline $\mathrm{J} 1908+0500$ & 0.291 & 201.42 & $227 / 7216$ & $0.30(0.05)$ & $0.64(0.03)$ & 16.41 & $-9.88(1.62)$ & 2.41 & 13.1 & 110.7 & 0.12 \\
\hline $\mathrm{J} 1909+0007$ & 1.017 & 112.79 & $151 / 2064$ & $0.40(0.23)$ & $0.78(0.11)$ & 1.38 & $-2.47(0.17)$ & 4.02 & 9.8 & 101.7 & 0.10 \\
\hline $\mathrm{J} 1909+1102$ & 0.284 & 149.98 & $251 / 7394$ & $0.44(0.05)$ & $0.67(0.03)$ & 12.96 & $-9.49(1.04)$ & 0.63 & 7.8 & 234.5 & 0.03 \\
\hline $\mathrm{J} 1910+0358$ & 2.330 & 82.93 & $16 / 901$ & $0.63(0.04)$ & $0.68(0.03)$ & 0.99 & $-4.41(1.10)$ & 0.28 & 9.2 & 34.0 & 0.27 \\
\hline $\mathrm{J} 1910+0714$ & 2.712 & 124.06 & $18 / 774$ & $0.80(0.06)$ & $0.62(0.06)$ & 0.60 & $-1.90(0.20)$ & 3.14 & 6.2 & 25.9 & 0.24 \\
\hline $\mathrm{J} 1910+0728$ & 0.325 & 283.70 & $248 / 6461$ & $0.48(0.05)$ & $0.68(0.04)$ & 11.56 & $-13.74(2.63)$ & 1.21 & 12.4 & 87.7 & 0.14 \\
\hline $\mathrm{J} 1910+1231$ & 1.442 & 258.64 & $53 / 1456$ & $0.58(0.04)$ & $0.73(0.04)$ & 0.78 & $-0.46(0.54)$ & 0.19 & 10.8 & 38.4 & 0.28 \\
\hline $\mathrm{J} 1913+0446$ & 1.616 & 109.10 & $46 / 1299$ & $0.49(0.05)$ & $0.77(0.05)$ & 1.52 & $-5.25(0.58)$ & 0.45 & 21.6 & 37.2 & 0.58 \\
\hline $\mathrm{J} 1913+1400$ & 0.521 & 145.05 & $60 / 4030$ & $0.86(0.03)$ & $0.65(0.03)$ & 2.16 & $-4.72(0.50)$ & 0.88 & 6.8 & 181.3 & 0.04 \\
\hline $\mathrm{J} 1914+0219$ & 0.458 & 233.80 & $77 / 4585$ & $0.46(0.04)$ & $0.75(0.03)$ & 3.90 & $-5.11(1.01)$ & 0.72 & 6.6 & 186.3 & 0.04 \\
\hline $\mathrm{J} 1915+0752$ & 2.058 & 105.30 & $15 / 1020$ & $0.62(0.07)$ & $0.89(0.07)$ & 1.47 & $-5.52(1.13)$ & 0.51 & 6.1 & 16.4 & 0.37 \\
\hline $\mathrm{J} 1915+1009$ & 0.405 & 241.69 & $406 / 5185$ & $0.84(0.05)$ & $0.64(0.04)$ & 5.83 & $-4.95(0.30)$ & 1.03 & 13.7 & 206.7 & 0.07 \\
\hline $\mathrm{J} 1916+1023$ & 1.618 & 329.80 & $29 / 1297$ & $0.71(0.11)$ & $0.97(0.14)$ & 3.13 & $-2.97(0.44)$ & 2.12 & 10.2 & 31.3 & 0.33 \\
\hline $\mathrm{J} 1916+1312$ & 0.282 & 237.01 & $62 / 7446$ & $0.95(0.02)$ & $0.48(0.02)$ & 3.31 & $-7.30(0.57)$ & 0.91 & 7.2 & 217.3 & 0.03 \\
\hline $\mathrm{J} 1917+0834$ & 2.130 & 29.18 & $72 / 985$ & $0.64(0.08)$ & $0.92(0.09)$ & 1.85 & $-3.49(0.95)$ & 0.43 & 13.6 & 42.5 & 0.32 \\
\hline $\mathrm{J} 1917+1353$ & 0.195 & 94.54 & $65 / 10769$ & $0.57(0.04)$ & $0.71(0.03)$ & 13.09 & $-7.56(0.57)$ & 0.61 & 6.5 & 272.3 & 0.02 \\
\hline $\mathrm{J} 1920+1040$ & 2.216 & 304.00 & $66 / 947$ & $0.31(0.08)$ & $0.91(0.09)$ & 1.40 & $-3.62(0.96)$ & 1.15 & 8.8 & 45.2 & 0.19 \\
\hline $\mathrm{J} 1932+1059$ & 0.227 & 3.18 & $825 / 9251$ & $3.09(0.08)$ & $0.86(0.09)$ & 1.07 & $-2.60(0.46)$ & 0.49 & 8.8 & 988.8 & 0.01 \\
\hline
\end{tabular}


Table 4.2: Correlation between the ratio of maximum single-pulse $\mathrm{S} / \mathrm{N}$ to FFT S/N and physical parameters of the pulsar, including the spin period, period derivative, DM, characteristic age, spin-down energy loss rate, magnetic field strength at the surface, and magnetic field strength at the light cylinder. From left to right we list the slope estimates, estimated standard deviations, and resulting reduced $\chi^{2}$ from linear fits to the data, as well as the significance of the slope. We also list the Spearman rank correlation coefficient and its P-value for a test of departure from zero.

\begin{tabular}{|c|c|c|c|c|c|}
\hline & \multicolumn{2}{|c|}{ Fit Statistics } & \multirow[t]{2}{*}{ Significance } & \multirow{2}{*}{$\begin{array}{l}\text { Correlation } \\
\text { Coefficient }\end{array}$} & \multirow{2}{*}{$\begin{array}{c}\text { Correlation } \\
\text { P-value }\end{array}$} \\
\hline & Slope & $\chi_{\text {red }}^{2}$ & & & \\
\hline$P$ & $0.70 \pm 0.07$ & 0.15 & $10 \hat{\sigma}$ & 0.54 & $7 \times 10^{-21}$ \\
\hline$\dot{P}$ & $0.05 \pm 0.03$ & 0.22 & $1 \hat{\sigma}$ & 0.10 & 0.11 \\
\hline DM & $0.08 \pm 0.09$ & 0.22 & None & 0.03 & 0.67 \\
\hline$\tau_{\mathrm{c}}$ & $0.15 \pm 0.03$ & 0.20 & $4 \hat{\sigma}$ & 0.25 & $4 \times 10^{-5}$ \\
\hline$\dot{E}$ & $-0.18 \pm 0.02$ & 0.17 & $8 \hat{\sigma}$ & -0.47 & $1 \times 10^{-15}$ \\
\hline$B_{\text {surf }}$ & $0.23 \pm 0.06$ & 0.21 & $3 \hat{\sigma}$ & 0.21 & $6 \times 10^{-4}$ \\
\hline$B_{\mathrm{LC}}$ & $-0.26 \pm 0.03$ & 0.16 & $9 \hat{\sigma}$ & -0.52 & $4 \times 10^{-19}$ \\
\hline
\end{tabular}


Table 4.3: Single-pulse detections of RRATs from the reprocessing of the PMPS. From left to right we list the RRAT name, spin period, DM, number of single pulses recorded out of the total number of stellar rotations during the observation, best-fit parameters from lognormal fits and the reduced $\chi^{2}$ of the fit, and peak single-pulse S/N. The fits were calculated through least-squares fitting of a scaled lognormal distribution, given by $\frac{C}{x \sigma \sqrt{2 \pi}} \exp \left(-\frac{(\ln (x)-\mu)^{2}}{2 \sigma^{2}}\right)$. The amplitude distributions for most RRATs is fit by two lognormal distributions, with the first distribution being consistent with noise, while the second distribution represents single pulses from the RRAT. Stars in the lognormal fit columns signify that we were unable to fit a lognormal function to that RRAT's amplitude distribution.

\begin{tabular}{|c|c|c|c|c|c|c|c|c|c|c|}
\hline \multirow[t]{2}{*}{ RRAT } & \multirow{2}{*}{$\begin{array}{l}\text { Period } \\
\text { (s) }\end{array}$} & \multirow{2}{*}{$\begin{array}{c}\mathrm{DM} \\
\left(\mathrm{pc} \mathrm{cm}^{-3}\right)\end{array}$} & \multirow{2}{*}{$\begin{array}{l}\text { Number of Single } \\
\text { Pulses Detected }\end{array}$} & \multicolumn{3}{|c|}{ First Lognormal } & \multicolumn{3}{|c|}{ Second Lognormal } & \multirow{2}{*}{$\begin{array}{c}\text { Max SP } \\
\text { S/N }\end{array}$} \\
\hline & & & & $\hat{\mu}(E /\langle E\rangle)$ & $\hat{\sigma}(E /\langle E\rangle)$ & $\chi_{\text {red }}^{2}$ & $\hat{\mu}(E /\langle E\rangle)$ & $\hat{\sigma}(E /\langle E\rangle)$ & $\chi_{\text {red }}^{2}$ & \\
\hline J0847-4316 & 5.977 & 292.50 & $4 / 351$ & $-8.8(40.7)$ & $2.82(7.21)$ & 1.12 & $1.53(0.01)$ & $0.11(0.01)$ & 0.52 & 10.77 \\
\hline $\mathrm{J} 1047-58$ & 1.231 & 69.30 & $17 / 1705$ & $*$ & $*$ & $*$ & $*$ & $*$ & $*$ & 23.65 \\
\hline J1317-5759 & 2.642 & 145.30 & $2 / 794$ & $-0.50(0.06)$ & $0.47(0.05)$ & 1.10 & $1.18(0.02)$ & $0.21(0.02)$ & 1.01 & 24.79 \\
\hline J1444-6026 & 4.759 & 367.70 & $2 / 441$ & $-0.58(0.27)$ & $0.72(0.26)$ & 1.58 & $1.60(0.02)$ & $0.20(0.02)$ & 0.38 & 42.58 \\
\hline $\mathrm{J} 1724-35$ & 1.422 & 554.90 & $3 / 1476$ & $0.45(0.96)$ & $1.87(2.35)$ & 3.28 & $1.47(0.03)$ & $0.27(0.03)$ & 1.08 & 12.69 \\
\hline J1754-3014 & 1.320 & 99.38 & $4 / 1590$ & $-0.10(0.11)$ & $0.69(0.16)$ & 2.54 & $1.21(0.02)$ & $0.28(0.02)$ & 0.62 & 9.60 \\
\hline J1819-1458 & 4.263 & 196.00 & $11 / 492$ & $-1.67(0.99)$ & $1.58(0.57)$ & 0.08 & $1.16(0.02)$ & $0.12(0.01)$ & 1.52 & 35.42 \\
\hline J1826-1419 & 0.771 & 160.00 & $2 / 2723$ & $0.48(0.04)$ & $0.72(0.04)$ & 3.34 & $*$ & $*$ & $*$ & 26.77 \\
\hline J1839-0136 & 0.933 & 307.00 & $2 / 2250$ & $0.50(0.05)$ & $0.82(0.04)$ & 2.18 & $*$ & $*$ & $*$ & 53.83 \\
\hline J1840-1419 & 6.598 & 19.40 & $69 / 318$ & $*$ & $*$ & $*$ & $0.69(0.04)$ & $0.15(0.02)$ & 5.34 & 27.53 \\
\hline $\mathrm{J} 1846-0257$ & 4.477 & 237.00 & $2 / 469$ & $-0.84(0.05)$ & $0.81(0.06)$ & 0.03 & $1.57(0.02)$ & $0.15(0.02)$ & 0.36 & 9.48 \\
\hline J1848-1247 & 0.414 & 88.00 & $3 / 5072$ & $*$ & $*$ & $*$ & $1.299(0.006)$ & $0.135(0.008)$ & 0.05 & 10.91 \\
\hline $\mathrm{J} 1911+0037$ & 6.940 & 100.00 & $1 / 302$ & $*$ & $*$ & $*$ & * & $*$ & $*$ & 9.23 \\
\hline J1913+0904 & 0.163 & 95.30 & $3 / 12883$ & $0.65(0.04)$ & $0.62(0.03)$ & 12.48 & $*$ & $*$ & $*$ & 7.73 \\
\hline $\mathrm{J} 1913+1330$ & 0.923 & 175.64 & $4 / 2275$ & $0.22(0.02)$ & $0.72(0.03)$ & 0.59 & $1.59(0.03)$ & $0.21(0.03)$ & 1.46 & 8.57 \\
\hline
\end{tabular}




\section{Chapter 5}

\section{Conclusions}

In this Chapter, we summarize our main results from the three main projects undertaken as part of this thesis and comment on possible future projects that arose as a result of these studies.

\subsection{The Crab Pulsar}

We used giant pulses from the Crab pulsar to probe both the normal and giant pulse emission from pulsars. Giant pulses at both $330 \mathrm{MHz}$ and $1.2 \mathrm{GHz}$ were compared with giant pulses recorded at $8.9 \mathrm{GHz}$ and $\gamma$-ray photons from the Fermi satellite. We found significant coincidences between $1.2 \mathrm{GHz}$ and $8.9 \mathrm{GHz}$ giant pulses at both the phase of the main pulse and interpulse. Even though the folded profile of the Crab pulsar differs greatly at these two frequencies, these results suggest that the emission mechanisms present are similar. A study of the distribution of giant pulse amplitudes revealed that giant pulse emission is most closely related to the emission of magnetars and RRATs.

We found no significant correlations between giant pulses and $\gamma$-ray photons. This indicates that increased pair production in the magnetosphere may contribute to giant pulse occurrence, although it is not a dominant factor. We did, however, find a $3 \sigma$ correlation at a time lag of one spin period. Previously, Shearer et al. (2003) 
found a $3 \%$ increase in the brightness of optical pulses at the time of GPs and Collins et al. (2012) found a slight correlation between GPs and enhanced optical pulses. Extended monitoring of the Crab is required to confirm or refute these results. Further studies of giant pulses from the Crab pulsar are being carried out at low frequencies in order to characterize pulse amplitudes and widths (Ellingson et al., 2013). During the span of our observations, a $\gamma$-ray flare from the Crab Nebula occurred. Based on a comparison of the folded profiles and average giant pulse profiles before and after the flare, we were able to rule out the pulsar as the cause of this flare. One theory as to the source of the flare is the 'jitter' model, where magnetic turbulence near a shock front causes very-high energy electrons to radiate high-energy photons (Teraki \& Takahara, 2013).

We note in passing that our observations made use of available time on the 43-m telescope. Our results on the Crab pulsar demonstrate that the 43-m telescope is still a very useful resource within the radio astronomical community. The system we developed for this study was subsequently used by Schmidt et al. (2013) to carry out searches for pulsars in 75 unidentified Northern VLA Sky Survey sources. We hope in future that further studies can be made using this system.

\subsection{New Pulsars from the Parkes Multibeam Pulsar Survey}

Our discovery of six new pulsars in the Parkes Multibeam Pulsar Survey indicates that there are a number of as-yet-undiscovered pulsars present in these data. These discoveries bring the total number of millisecond pulsars found in the survey 
to 26. With the enormous number of pulsar candidates output by searches like this, automated ranking is the most efficient way to reduce the number of candidates to be viewed. Keith et al. (2009) found that weak pulsars and pulsars with high DMs were ranked highly by automated searches. However, most of these pulsars have long periods, i.e. periods on the order of hundreds of milliseconds. As the ratio of

DM to period increases, the detected pulse profile is significantly broadened and begins to look more sinusoidal. These candidates are harder to select via ranking systems. Eatough et al. (2010) found that artificial neural networks have difficulty detecting short period pulsars, with their own neural network detecting only $50 \%$ of pulsars with periods less than $10 \mathrm{~ms}$. We plan to follow up and confirm a number of our candidates from this search. Timing observations of the currently confirmed pulsars, when scheduled, will no doubt result in a number of interesting results and possibly provide further millisecond pulsars for use in Pulsar Timing Arrays. Continued timing observations of PSR J1725-3853 and PSR J1753-2822 will allow us to further improve their timing solutions.

\subsection{Single-pulse Studies of the PMPS}

Given the long observation times of the PMPS, we were able to record more single pulses from pulsars and RRATs, allowing us to put better constraints on the amplitude distributions. Classifying these distributions, which we fit with lognormal distributions and power-law tails, can help us to understand the connection between pulsars and RRATs and the pulsar emission mechanism. 
Periodicity searches allowed us to calculate the ratio of the maximum singlepulse $\mathrm{S} / \mathrm{N}$ to the FFT $\mathrm{S} / \mathrm{N}$, with resulting values from 0.01 to 1.51 . These values are consistent with estimates by Deneva et al. (2009), with a few outliers possibly due to longer observation times. The fact that most pulsars have ratios less than one shows that most pulsars are more easily detectable in a periodicity search versus a single pulse search.

We compared these ratios to physical properties of each pulsar. We found strong correlations between the ratio and a few parameters, the strongest of these being the spin period. The other strong correlations were determined to be dominated by this spin period correlation. Previous studies (Burke-Spolaor et al., 2012) found similar strong correlations between modulation and some physical parameters, but these were found to be caused by a strong anticorrelation between the modulation index and integrated $\mathrm{S} / \mathrm{N}$.

For most of the RRATs, their energy distributions are similar to those for our 'bursty' pulsars, but the 'bump' is much more pronounced, and there seems to be two distinct distributions. The estimated means of the first distributions are near zero, suggesting they are consistent with noise. The values of $\hat{\mu}$ and $\hat{\sigma}$ for the second distributions are consistent with those from the known pulsars, with a smaller range.

Further investigations of the survey results are now underway to quantify the existence of other pulses in the data. The recent discoveries of Fast Radio Bursts with high dispersion measures (Lorimer et al., 2007; Keane et al., 2012; Thornton et al., 2013) point to the presence of an entirely new class of compact sources whose origin remains unknown. 


\section{Bibliography}

Abdo A. A., Ackermann M., Ajello M., Atwood W. B., Axelsson M., Baldini L., Ballet J., Barbiellini G., Baring M. G., Bastieri D., Bechtol K., Bellazzini R., Berenji B., Blandford R. D., Bloom E. D., 2010, ApJ, 708, 1254

Allen C. W., 1973, Astrophysical quantities

Antoniadis J., Freire P. C. C., Wex N., Tauris T. M., Lynch R. S., van Kerkwijk M. H., Kramer M., Bassa C., Dhillon V. S., Driebe T., Hessels J. W. T., 2013, Science, 340, 448

Argyle E., Gower J. F. R., 1972, ApJ, 175, L89

Atwood W. B., Abdo A. A., Ackermann M., Althouse W., Anderson B., Axelsson M., Baldini L., Ballet J., Band D. L., Barbiellini G., et al. 2009, ApJ, 697, 1071

Baade W., Zwicky F., 1934, Proc. Nat. Acad. Sci., 20, 259

Bednarek W., Idec W., 2011, MNRAS, 414, 2229

Bhat N. D. R., Tingay S. J., Knight H. S., 2008, ApJ, 676, 1200

Bhat N. D. R., Wayth R. B., Knight H. S., Bowman J. D., Oberoi D., Barnes D. G., Briggs F. H., Cappallo R. J., Herne D., Kocz J., Lonsdale C. J., Lynch M. J., Stansby B., Stevens J., Torr G., Webster R. L., Wyithe J. S. B., 2007, ApJ, 665, 618

Bhattacharya D., van den Heuvel E. P. J., 1991, Phys. Rep., 203, 1

Bietenholz M. F., Kassim N., Frail D. A., Perley R. A., Erickson W. C., Hajian A. R., 1997, ApJ, 490, 291

Biggs J. D., 1992, ApJ, 394, 574

Bilous A. V., Kondratiev V. I., McLaughlin M. A., Ransom S. M., Lyutikov M., Mickaliger M., Langston G. I., 2011, ApJ, 728, 110

Bisnovatyi-Kogan G. S., Komberg B. V., 1974, Soviet Astronomy, 18, 217

Burgay M., D’Amico N., Possenti A., Manchester R. N., Lyne A. G., Joshi B. C., McLaughlin M. A., Kramer M., Sarkissian J. M., Camilo F., Kalogera V., Kim C., Lorimer D. R., 2003, Nature, 426, 531

Burke-Spolaor S., Johnston S., Bailes M., Bates S. D., Bhat N. D. R., Burgay M., Champion D. J., D’Amico N., Keith M. J., Kramer M., Levin L., Milia S., Possenti A., Stappers B., van Straten W., 2012, MNRAS, 423, 1351

Bykov A. M., Pavlov G. G., Artemyev A. V., Uvarov Y. A., 2012, MNRAS, 421, L67 
Cairns I. H., Johnston S., Das P., 2001, ApJ, 563, L65

Cairns I. H., Johnston S., Das P., 2004, MNRAS, 353, 270

Cerutti B., Uzdensky D. A., Begelman M. C., 2012, ApJ, 746, 148

Cognard I., Shrauner J. A., Taylor J. H., Thorsett S. E., 1996, ApJ, 457, L81

Collins S., Shearer A., Stappers B., Barbieri C., Naletto G., Zampieri L., Verroi E., Gradari S., 2012, in Griffin E., Hanisch R., Seaman R., eds, IAU Symposium Vol. 285 of IAU Symposium, Crab Pulsar: Enhanced Optical Emission During Giant Radio Pulses. pp 296-298

Cordes J. M., Bhat N. D. R., Hankins T. H., McLaughlin M. A., Kern J., 2004, ApJ, 612, 375

Cordes J. M., Lazio T. J. W., 2002, ArXiv e-prints

Cordes J. M., Lazio T. J. W., McLaughlin M. A., 2004, New Astron. Rev., 48, 1459

Cordes J. M., McLaughlin M. A., 2003, ApJ, 596, 1142

Cordes J. M., Shannon R. M., 2008, ApJ, 682, 1152

Demorest P. B., Pennucci T., Ransom S. M., Roberts M. S. E., Hessels J. W. T., 2010, Nature, 467, 1081

Deneva J. S., Cordes J. M., McLaughlin M. A., Nice D. J., Lorimer D. R., Crawford F., Bhat N. D. R., Camilo F., Champion D. J., Freire P. C. C., Edel S., Kondratiev V. I., Hessels J. W. T., 2009, ApJ, 703, 2259

DuPlain R., Ransom S., Demorest P., Brandt P., Ford J., Shelton A. L., 2008, in Society of Photo-Optical Instrumentation Engineers (SPIE) Conference Series Vol. 7019 of Society of Photo-Optical Instrumentation Engineers (SPIE) Conference Series, Launching GUPPI: the Green Bank Ultimate Pulsar Processing Instrument

Eatough R. P., Keane E. F., Lyne A. G., 2009, MNRAS, 395, 410

Eatough R. P., Kramer M., Lyne A. G., Keith M., 2011, in Burgay M., D’Amico N., Esposito P., Pellizzoni A., Possenti A., eds, American Institute of Physics Conference Series Vol. 1357 of American Institute of Physics Conference Series, Results from the latest re-analysis of the Parkes multi-beam pulsar survey. pp $58-59$

Eatough R. P., Molkenthin N., Kramer M., Noutsos A., Keith M. J., Stappers B. W., Lyne A. G., 2010, MNRAS, 407, 2443

Ellingson S. W., Clarke T. E., Craig J., Hicks B. C., Lazio T. J. W., Taylor G. B., Wilson T. L., Wolfe C. N., 2013, ApJ, 768, 136 
Espinoza C. M., Jordan C., Stappers B. W., Lyne A. G., Weltevrede P., Cognard I., Theureau G., 2010, The Astronomer's Telegram, 2889, 1

Faucher-Giguère C.-A., Kaspi V. M., 2006, ApJ, 643, 332

Faulkner A. J., Stairs I. H., Kramer M., Lyne A. G., Hobbs G., Possenti A., Lorimer D. R., Manchester R. N., McLaughlin M. A., D’Amico N., Camilo F., Burgay M., 2004, MNRAS, 355, 147

Ferdman R. D., van Haasteren R., Bassa C. G., Burgay M., Cognard I., Corongiu A., D’Amico N., Desvignes G., Hessels J. W. T., Janssen G. H., Jessner A., Jordan C., Karuppusamy R., Keane E. F., 2010, Classical and Quantum Gravity, 27, 084014

Glendenning N., 1996, Compact Stars. Nuclear Physics, Particle Physics and General Relativity.

Gold T., 1968, Nature, 218, 731

Goldreich P., Julian W. H., 1969, ApJ, 157, 869

Hankins T. H., Eilek J. A., 2007, ApJ, 670, 693

Hankins T. H., Kern J. S., Weatherall J. C., Eilek J. A., 2003, Nature, 422, 141

Hays E., Buehler R., D'Ammando F., Grove J. E., Ray P. S., 2010, The Astronomer's Telegram, 2879, 1

Hessels J. W. T., Ransom S. M., Stairs I. H., Freire P. C. C., Kaspi V. M., Camilo F., 2006, Science, 311, 1901

Hewish A., Bell S. J., Pilkington J. D. H., Scott P. F., Collins R. A., 1968, Nature, 217,709

Hobbs G., Archibald A., Arzoumanian Z., Backer D., Bailes M., Bhat N. D. R., Burgay M., Burke-Spolaor S., Champion D., Cognard I., Coles W., Cordes J., Demorest P., Desvignes G., Ferdman R. D., 2010, Classical and Quantum Gravity, 27,084013

Hobbs G., Faulkner A., Stairs I. H., Camilo F., Manchester R. N., Lyne A. G., Kramer M., D’Amico N., Kaspi V. M., Possenti A., McLaughlin M. A., Lorimer D. R., Burgay M., Joshi B. C., Crawford F., 2004, MNRAS, 352, 1439

Hotan A. W., van Straten W., Manchester R. N., 2004, PASA, 21, 302

Istomin Y. N., 2004, in Camilo F., Gaensler B. M., eds, Young Neutron Stars and Their Environments Vol. 218 of IAU Symposium, Origin of Giant Radio Pulses. p. 369

Jackson J. D., 1962, Classical Electrodynamics 
Jenet F., Finn L. S., Lazio J., Lommen A., McLaughlin M., Stairs I., Stinebring D., Verbiest J., Archibald A., Arzoumanian Z., Backer D., Cordes J., 2009, ArXiv e-prints

Jessner A., Popov M. V., Kondratiev V. I., Kovalev Y. Y., Graham D., Zensus A., Soglasnov V. A., Bilous A. V., Moshkina O. A., 2010, A\&A, 524, A60

Johnston S., Romani R. W., 2002, MNRAS, 332, 109

Johnston S., van Straten W., Kramer M., Bailes M., 2001, ApJ, 549, L101

Jouteux S., Ramachandran R., Stappers B. W., Jonker P. G., van der Klis M., 2002, A\&A, 384, 532

Karuppusamy R., Stappers B. W., Lee K. J., 2012, A\&A, 538, A7

Karuppusamy R., Stappers B. W., Serylak M., 2011, A\&A, 525, A55

Karuppusamy R., Stappers B. W., van Straten W., 2010, A\&A, 515, A36

Katayama T., Miyatsu T., Saito K., 2012, ApJS, 203, 22

Keane E. F., Kramer M., Lyne A. G., Stappers B. W., McLaughlin M. A., 2011, MNRAS, 415, 3065

Keane E. F., Ludovici D. A., Eatough R. P., Kramer M., Lyne A. G., McLaughlin M. A., Stappers B. W., 2010, MNRAS, 401, 1057

Keane E. F., McLaughlin M. A., 2011, Bulletin of the Astronomical Society of India, 39,333

Keane E. F., Stappers B. W., Kramer M., Lyne A. G., 2012, MNRAS, 425, L71

Keith M. J., Eatough R. P., Lyne A. G., Kramer M., Possenti A., Camilo F., Manchester R. N., 2009, MNRAS, 395, 837

Keith M. J., Jameson A., van Straten W., Bailes M., Johnston S., Kramer M., Possenti A., Bates S. D., Bhat N. D. R., Burgay M., Burke-Spolaor S., 2010, MNRAS, 409, 619

Knight H. S., Bailes M., Manchester R. N., Ord S. M., Jacoby B. A., 2006, ApJ, 640,941

Knispel B., Eatough R. P., Kim H., Keane E. F., Allen B., Anderson D., Aulbert C., Bock O., Crawford F., Eggenstein H.-B., Fehrmann H., 2013, ArXiv e-prints

Kramer M., Bell J. F., Manchester R. N., Lyne A. G., Camilo F., Stairs I. H., D’Amico N., Kaspi V. M., Hobbs G., Morris D. J., Crawford F., Possenti A., 2003, MNRAS, 342, 1299

Kramer M., Johnston S., van Straten W., 2002, MNRAS, 334, 523 
Kramer M., Stairs I. H., Manchester R. N., McLaughlin M. A., Lyne A. G., Ferdman R. D., Burgay M., Lorimer D. R., Possenti A., D’Amico N., 2006, Science, 314, 97

Kuz'min A. D., Belyatsky Y. A., Dumsky D. V., Izvekova V. A., Lapaev K. A., Logvinenko S. V., Losovsky B. Y., Pugachev V. D., 2011, Astronomy Reports, 55,416

Langston G., 2007, Highlights of Astronomy, 14, 367

Li X.-D., 2006, ApJ, 646, L139

Lorimer D. R., 2008, Living Reviews in Relativity, 11, 8

Lorimer D. R., Bailes M., McLaughlin M. A., Narkevic D. J., Crawford F., 2007, Science, 318,777

Lorimer D. R., Faulkner A. J., Lyne A. G., Manchester R. N., Kramer M., McLaughlin M. A., Hobbs G., Possenti A., Stairs I. H., Camilo F., 2006, MNRAS, 372, 777

Lorimer D. R., Kramer M., 2004, Handbook of Pulsar Astronomy

Lorimer D. R., Yates J. A., Lyne A. G., Gould D. M., 1995, MNRAS, 273, 411

Lundgren S. C., 1994, PhD thesis, Cornell Univ., Ithaca, NY.

Lundgren S. C., Cordes J. M., Ulmer M., Matz S. M., Lomatch S., Foster R. S., Hankins T., 1995, ApJ, 453, 433

Luo Q., Melrose D., 2007, MNRAS, 378, 1481

Lyne A. G., Burgay M., Kramer M., Possenti A., Manchester R. N., Camilo F., McLaughlin M. A., Lorimer D. R., D’Amico N., Joshi B. C., Reynolds J., Freire P. C. C., 2004, Science, 303, 1153

Lyne A. G., Mankelow S. H., Bell J. F., Manchester R. N., 2000, MNRAS, 316, 491

Lyne A. G., Pritchard R. S., Graham-Smith F., 1993, MNRAS, 265, 1003

Lyutikov M., 2007, MNRAS, 381, 1190

Lyutikov M., Blandford R. D., Machabeli G., 1999, MNRAS, 305, 338

Machabeli G. Z., Usov V. V., 1979, Soviet Astronomy Letters, 5, 238

Majid W. A., Naudet C. J., Lowe S. T., Kuiper T. B. H., 2011, ApJ, 741, 53

Manchester R. N., Hobbs G., Bailes M., Coles W. A., van Straten W., Keith M. J., Shannon R. M., Bhat N. D. R., Brown A., Burke-Spolaor S. G., Champion D. J., Chaudhary A., Edwards R. T., 2013, PASA, 30, 17 
Manchester R. N., Lyne A. G., Camilo F., Bell J. F., Kaspi V. M., D’Amico N., McKay N. P. F., Crawford F., Stairs I. H., Possenti A., Kramer M., Sheppard D. C., 2001, MNRAS, 328, 17

McLaughlin M. A., Cordes J. M., 2003, ApJ, 596, 982

McLaughlin M. A., Lyne A. G., Keane E. F., Kramer M., Miller J. J., Lorimer D. R., Manchester R. N., Camilo F., Stairs I. H., 2009, MNRAS, 400, 1431

McLaughlin M. A., Lyne A. G., Lorimer D. R., Kramer M., Faulkner A. J., Manchester R. N., Cordes J. M., Camilo F., Possenti A., Stairs I. H., Hobbs G., D'Amico N., Burgay M., O’Brien J. T., 2006, Nature, 439, 817

Miller J., McLaughlin M., Rea N., Keane E., Lyne A., Kramer M., Manchester R., Lazaridis K., 2011, in Burgay M., D'Amico N., Esposito P., Pellizzoni A., Possenti A., eds, American Institute of Physics Conference Series Vol. 1357 of American Institute of Physics Conference Series, Multiwavelength Studies of Rotating Radio Transients. pp 161-164

Moffett D. A., 1997, PhD thesis, , New Mexico Inst. Mining and Technology, (1997)

Moffett D. A., Hankins T. H., 1996, ApJ, 468, 779

Morris D. J., Hobbs G., Lyne A. G., Stairs I. H., Camilo F., Manchester R. N., Possenti A., Bell J. F., Kaspi V. M., Amico N. D., McKay N. P. F., Crawford F., Kramer M., 2002, MNRAS, 335, 275

Pacini F., 1968, Nature, 219, 145

Parsons A., Werthimer D., Backer D., Bastian T., Bower G., Brisken W., Chen H., Deller A., Filiba T., Gary D., Greenhill L., Hawkins D., 2009, in astro2010: The Astronomy and Astrophysics Decadal Survey Vol. 2010 of Astronomy, Digital Instrumentation for the Radio Astronomy Community. p. 21

Perna R., Soria R., Pooley D., Stella L., 2008, MNRAS, 384, 1638

Petrova S. A., 2006, Chinese Journal of Astronomy and Astrophysics Supplement, 6, 020000

Petrova S. A., 2009, MNRAS, 395, 1723

Popov M., Soglasnov V., Kondratiev V., Bilous A., Moshkina O., Oreshko V., Ilyasov Y., Sekido M., Kondo T., 2009, PASJ, 61, 1197

Popov M. V., Soglasnov V. A., Kondrat'ev V. I., Bilous A. V., Sazankov S. V., Smirnov A. I., Kanevskii B. Z., Oreshko V. V., Ilyasov Y. P., 2008, Astronomy Reports, 52, 900

Popov M. V., Soglasnov V. A., Kondrat'Ev V. I., Kostyuk S. V., Ilyasov Y. P., Oreshko V. V., 2006, Astronomy Reports, 50, 55 
Popov M. V., Stappers B., 2007, A\&A, 470, 1003

Press W. H., 1986, in Hut P., McMillan S. L. W., eds, The Use of Supercomputers in Stellar Dynamics Vol. 267 of Lecture Notes in Physics, Berlin Springer Verlag, Techniques and Tricks for N-Body Computation. p. 184

Rankin J. M., Comella J. M., Craft Jr. H. D., Richards D. W., Campbell D. B., Counselman III C. C., 1970, ApJ, 162, 707

Ransom S. M., Cordes J. M., Eikenberry S. S., 2003, ApJ, 589, 911

Ransom S. M., Eikenberry S. S., Middleditch J., 2002, AJ, 124, 1788

Ransom S. M., Greenhill L. J., Herrnstein J. R., Manchester R. N., Camilo F., Eikenberry S. S., Lyne A. G., 2001, ApJ, 546, L25

Rickett B. J., Lyne A. G., 1990, MNRAS, 244, 68

Ritchings R. T., 1976, MNRAS, 176, 249

Scargle J. D., 1982, ApJ, 263, 835

Schmidt D., Crawford F., Langston G., Gilpin C., 2013, AJ, 145, 116

Schwarzschild B., 2011, Physics Today, 64, 010000

Serylak M., Stappers B. W., Weltevrede P., Kramer M., Jessner A., Lyne A. G., Jordan C. A., Lazaridis K., Zensus J. A., 2009, MNRAS, 394, 295

Shearer A., Stappers B., O'Connor P., Golden A., Strom R., Redfern M., Ryan O., 2003, Science, 301, 493

Shibazaki N., Murakami T., Shaham J., Nomoto K., 1989, Nature, 342, 656

Smirnova T. V., Logvinenko S. V., 2009, Astronomy Reports, 53, 334

Staelin D. H., Reifenstein III E. C., 1968, Science, 162, 1481

Staveley-Smith L., Wilson W. E., Bird T. S., Disney M. J., Ekers R. D., Freeman K. C., Haynes R. F., Sinclair M. W., Vaile R. A., Webster R. L., Wright A. E., 1996, PASA, 13, 243

Stephenson F. R., Green D. A., 2002, Historical supernovae and their remnants, by F. Richard Stephenson and David A. Green. International series in astronomy and astrophysics, vol. 5. Oxford: Clarendon Press, 2002, ISBN 0198507666, 5

Tavani M., Bulgarelli A., Vittorini V., Pellizzoni A., Striani E., Caraveo P., Weisskopf M. C., Tennant A., Pucella G., Trois A., Costa E., Evangelista Y., Pittori C., Verrecchia F., Del Monte E., Campana R., 2011, Science, 331, 736 
Taylor J. H., 1992, Philosophical Transactions of the Royal Society of London, 341, 117-134 (1992), 341, 117

Taylor J. H., Weisberg J. M., 1982, ApJ, 253, 908

Taylor J. H., Weisberg J. M., 1989, ApJ, 345, 434

Teraki Y., Takahara F., 2013, ApJ, 763, 131

Thornton D., Stappers B., Bailes M., Barsdell B. R., Bates S. D., Bhat N. D. R., 2013, ArXiv e-prints

Timokhin A. N., 2010, MNRAS, 408, L41

van Straten W., Bailes M., Britton M., Kulkarni S. R., Anderson S. B., Manchester R. N., Sarkissian J., 2001, Nature, 412, 158

Weatherall J. C., 1998, ApJ, 506, 341

Weltevrede P., Stappers B. W., Rankin J. M., Wright G. A. E., 2006, ApJ, 645, L149

Zhuravlev V. I., Popov M. V., Kondrat'ev V. I., Kovalev Y. Y., Ghigo F., Soglasnov V. A., 2011, Astronomy Reports, 55, 724 